National Library

of Canada

Acquisitions and

Bibliographic Services Branch

395 Wellington Street

Ottawa. Ontaro

KIA ONA
Bibliotheque nationale

du Canada

Direction des acquisitions et

des services bibliographiques

395. rue Wellington

Ottawa (Ontario)

KIAON
NOTICE

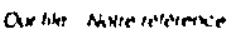

AVIS
The quality of this microform is heavily dependent upon the quality of the original thesis submitted for microfilming. Every effort has been made to ensure the highest quality of reproduction possible.

If pages are missing, contact the university which granted the degree.

Some pages may have indistinct print especially if the original pages were typed with a poor typewriter ribbon or if the university sent us an infericr photocopy.
La qualité de cette microforme dépend grandement de la qualité de la thèse soumise au microfilmage. Nous avons tout fait pour assurer une qualité supérieure de reproduction.

S'il manque des pages, veuillez communiquer avec l'université qui a conféré le grade.

La qualité d'impression de certaines pages peut laisser à désirer, surłout si les pages originales ont été dactylographiées à l'aide d'un ruban usé ou si l'université nous a fait parvenir une photocopie de qualité inférieure.

La reproduction, même partielle, de cette microforme est soumise à la Loi canadienne sur le droit d'auteur, SRC 1970, c. C-30, et ses amendements subséquents.
Reproduction in full or in part of this microform is governed by the Canadian Copyright Act, R.S.C. 1970, c. C-30, and subsequent amendments.

Canadäa 


\title{
The Design of Log-Domain Filters Based On the Operational Simulation of LC Ladders
}

\author{
by \\ Douglas Perry
}

Department of Electrical Engineering

McGill University, Montreal

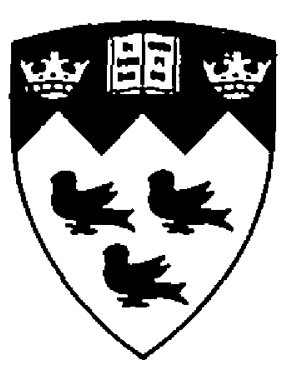

March 1995

A Thesis submitted to the Faculty of Graduate Studies and Research in partial

fulfitlment of the requirements for the degree of Master of Engineering

(C) Douglas Perry, 1995 
National Library

of Canada

Acquisitions and

Bibliographic Services Branch

395 Wellington Street

Ottawa. Ontario

K1AON4
Bibliotheque nationale

du Canada

Direction des acquisitions et

des services bibliographiques

395, ne Wellinglon

Ottawa (Ontario)

K1A ON4

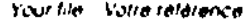

Ow ble Novie coturence
THE AUTHOR HAS GRANTED AN IRREVOCABLE NON-EXCLUSIVE LICENCE ALLOWING THE NATIONAL LIBRARY OF CANADA TO REPRODUCE, LOAN, DISTRIBUTE OR SELL COPIES OF HIS/HER THESIS BY ANY MEANS AND IN ANY FORM OR FORMAT, MAKING THIS THESIS AVAILABLE TO INTERESTED PERSONS.
L'AUTEUR A ACCORDE UNE LICENCE IRREVOCABLE ET NON EXCLUSIVE PERMETTANT A LA BIBLIOTHEQUE NATIONALE DU CANADA DE REPRODUIRE, PRETER, DISTRIBUER OU VENDRE DES COPIES DE SA THESE DE QUELQUE MANIERE E'T SOUS QUELQUE FORME QUE CE SOIT POUR METTRE DES EXEMPLAIRES DE CETTE THESE A LA DISPOSITION DES PERSONNE INTERESSEES.
THE AUTHOR RETAINS OWNERSHIP OF THE COPYRIGHT IN HIS/HER THESIS. NEITHER THE THESIS NOR SUBSTANTIAL EXTRACTS FROM IT MAY BE PRINTED OR OTHERWISE REPRODUCED WITHOUT HIS/HER PERMISSION.
L'AUTEUR CONSERVE LA PROPRIETE DU DROIT D'AUTEUR QUI PROTEGE SA THESE. NI LA THESE NI DES EXTRAITS SUBSTANTIELS DE CELLECI NE DOIVENT ETRE IMPRIMES OU AUTREMENT REPRODUITS SANS SON AUTORISATION. 


\section{Abstract}

With the advent of portable communications there has been an increased emphasis on high-speed, low-power microelectronics. The log-domain filter is a novel form of continuous-time filter which shows promise in this area. Based on the translinear principle, log-domain filters are simple in structure, fast and easily tunable.

This thesis will present a technique for the design of log-domain filters which is based on the operational simulation of LC ladders. The technique is demonstrated through the complete design of a fifth-order Chebyshev log-domain filter, as well as the design of elliptic and bandpass filters. The filters were fabricated using the Gennum GA911 bipolar transistor array technology. HSPICE simulation and experimental results are shown with emphasis on frequency behavior and linearity. The filters showed good correlation between the measured response and the original specifications. They were tunable over two decades and could be operated up to $1 \mathrm{MHz}$ or $1 / 10$ th of the $f_{T}$ of the slowest transistor. Total harmonic distortion and intermodulation distortion was measured with results ranging from $-45 \mathrm{~dB}$ to $-70 \mathrm{~dB}$. 


\section{Résumé}

La popularité des communications mobiles génère un intérêt grandissant pour les circuits électroniques à grande vitesse et à faible puissancc. Les "filtres logarithmiques" sont des circuits récemment développés et prometteurs dans ce domaine. Ce sont des circuits simples, $r i$; des et facilement réglables.

Cette thèse présente une nouvelle méthode pour la conception des filtres logarithmiques basée sur la simulation de circuits passifs. A titre d'excmple, un filtre Chebyshev ainsi qu'un filtre élliptique, tous deux de cinquième ordre, ont été conçus. Les deux circuits, fabriqués à l'aide du procédé GA911 de la compagnie Gennum, démontrent des résultats expérimentaux comparables à ceux de simulations effectuées avec le logiciel HSPICE. Leur réponse en fréquence est réglable sur un intervale de deux décades et ils fonctionnent jusqu'à $1 \mathrm{MHz}$, soit un dixième de $f_{T}$ pour le transistor le plus lent. De plus, ils se montrent très linéaires avec des mesures de distorsion entre $-45 \mathrm{~dB}$ et $-70 \mathrm{~dB}$. 


\section{Acknowledgements}

At this time, I would like to acknowledge the many people who made this thesis possible. First and foremost, I extend a sincere thank you to my supervisor, Gordon Roberts, for his ideas, his enthusiasm and his constant willingness to spare a few minutes for his students.

I want to thank my Mom and Dad for all their love, support and good advice. Thanks to my brother Curt, for his simple outlook on life and unequivocal devotion. And a very special thanks to Anoush, who was always there for me.

I am deeply grateful to the many members of the MACS lab who were willing to answer my questions no matter how ridiculous, and to Jacek Slaboszewicz who kept the lab running. Thanks to all my friends, especially Homer, Dave and Danny, for keeping the wheels spinning.

Finally, I would like to thank Gennum for providing the silicon which allowed me to implement my ideas and the people at CMC for their technical support. This work was made possible thanks to the financial support of NSERC and the Micronet. 


\section{Table of Contents}

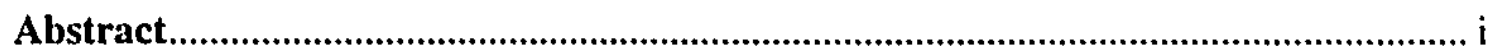

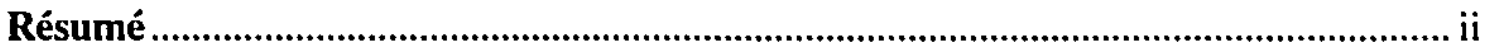

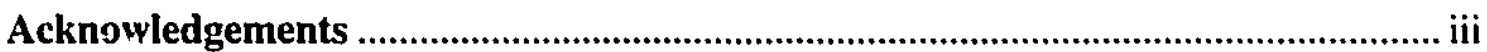

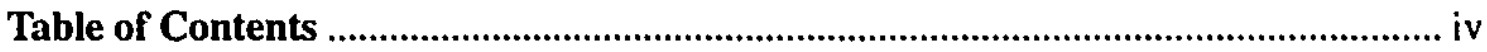

List of Figures................................................................................................................ viii

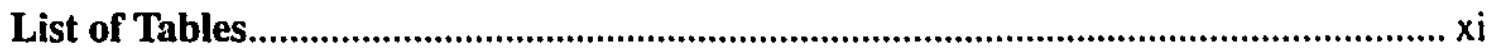

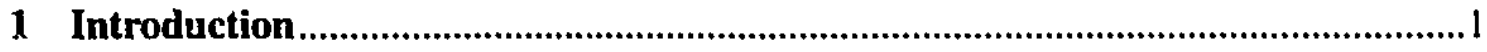

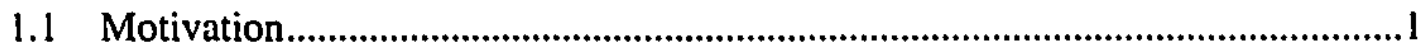

1.2 Conventional Integrated Continuous-Time Filters ..................................................

1.2 .1 MOS-C Filters ...............................................................................................

1.2.2 Transconductance-C (gm-C) Filters ............................................................4

1.3 A Novel Approach to Continuous-Time Filtering: The Log-Domain Filter ........6

1.4 The Operational Simulation of LC Ladders ...........................................................

1.5 Thesis Outline 
2 The Basic I,og-Domain Building Blocks

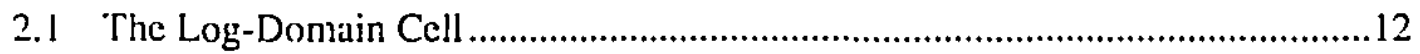

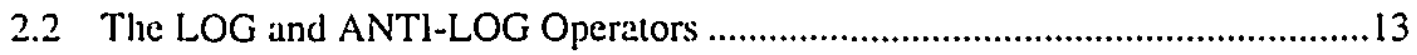

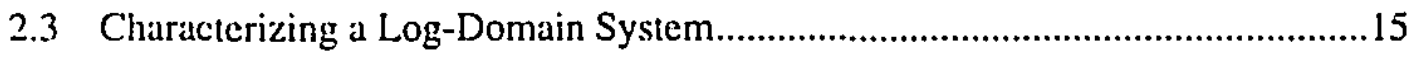

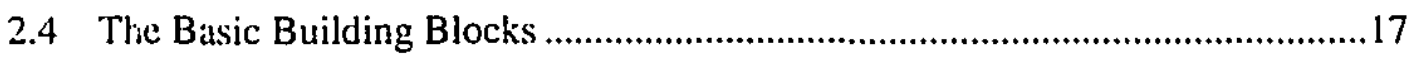

2.4.1 The Non-Inverting Integrator.............................................................. 17

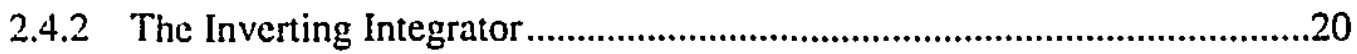

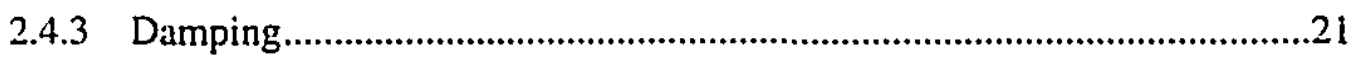

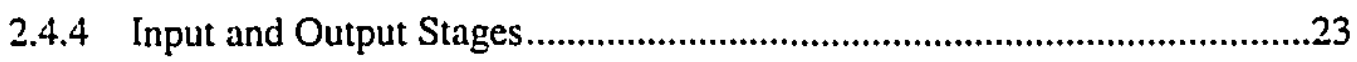

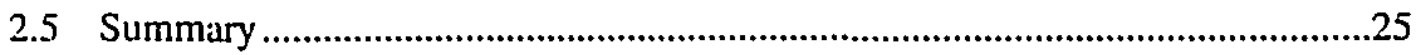

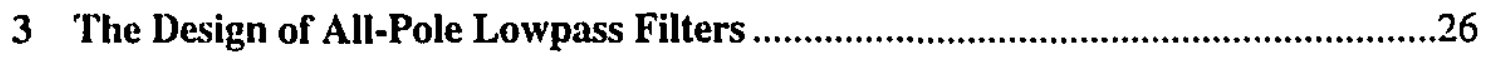

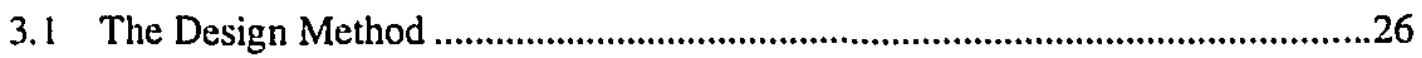

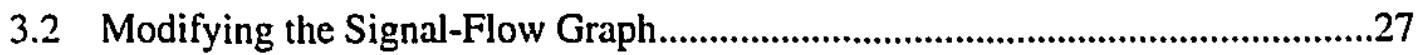

3.3 A Fifth-Order Chebyshev Filter Design Example .........................................29

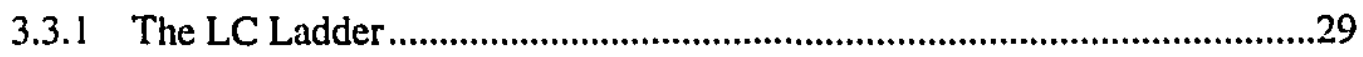

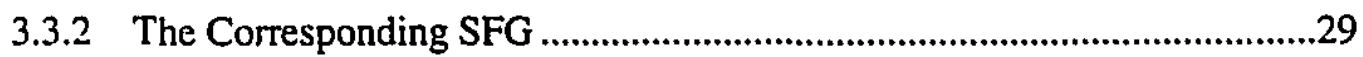

3.3.3 The Complete Chebyshev Log-Domain Filter...........................................32

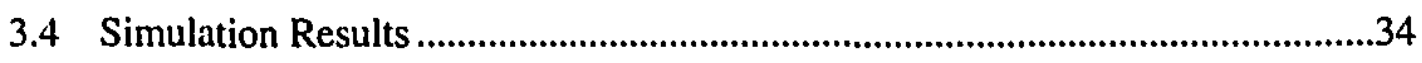

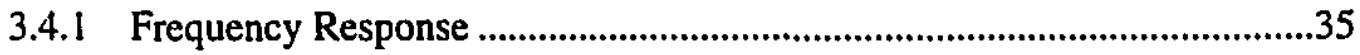

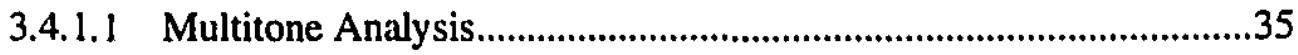

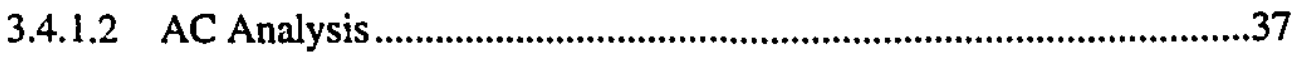

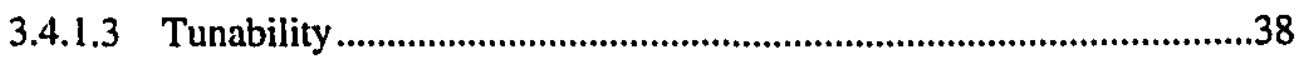

3.4.1.4 High-Frequency Performance.............................................................39

3.4.2 Distortion Measurements .......................................................................40

3.4.2.1 The Intercept Concept.................................................................41

3.4.2.2 Total Harmonic Distortion..............................................................42

3.4.2.3 Intermodulation Distortion........................................................44 


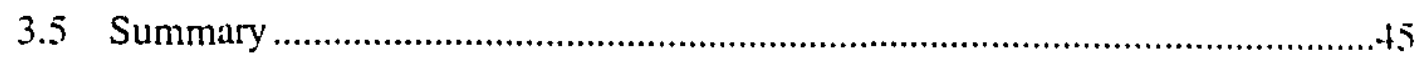

4 Elliptic and Bandpass Filters .........................................................................

4.1 Elliptic Filters ............................................................................................

4.1.1 Derivation of a Third-Order Elliptic Filter ..............................................to

4.1.2 Simulation Results ............................................................................50

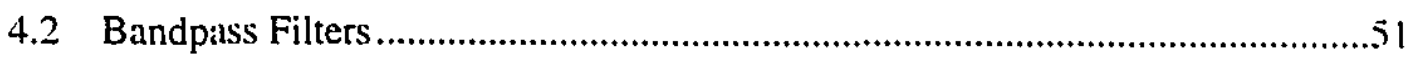

4.2.1 Derivation of a Fourth-Order Log-Domain Bandpass Filter .....................51

4.2.2 Simulation Results .................................................................................

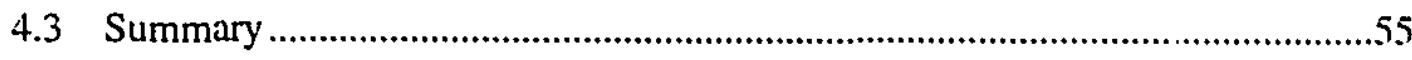

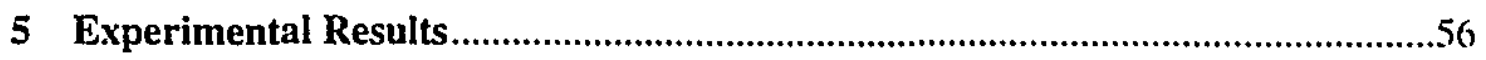

5.1 The Biquadratic Filter .................................................................................56

5.1.1 The Test Set-Up .....................................................................................57

5.1.2 Frequency Performance ……..............................................................5

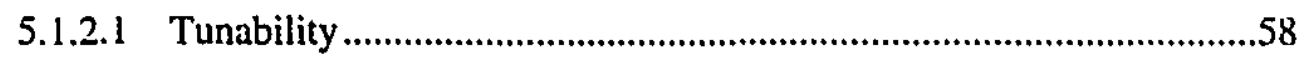

5.1.2.2 High-frequency operation ................................................................59

5.1.3 Linearity Measurements.............................................................................60

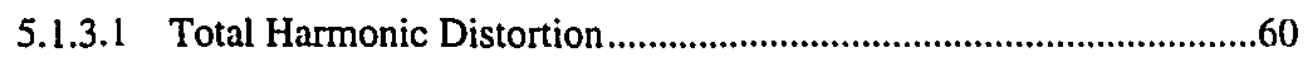

5.1.3.2 Intermodulation Distortion.........................................................60

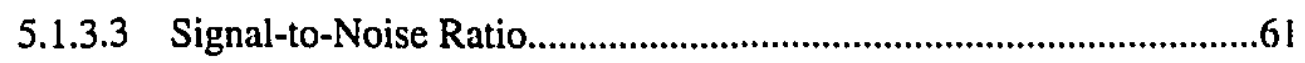

5.2 The Fifth-Order Chebyshev Filter ......................................................................62

5.2.1 The Test Set-up .............................................................................................63

5.2.2 Frequency Performance ...........................................................................63

5.2.3 Linearity Measurements.......................................................................65

5.2.3.1 Total Harmonic Distortion ..........................................................65

5.2.3.2 Intermodulation Distortion...........................................................66

5.3 The Log-Domain Elliptic Filter ..........................................................................67

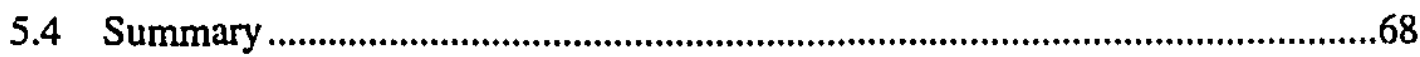




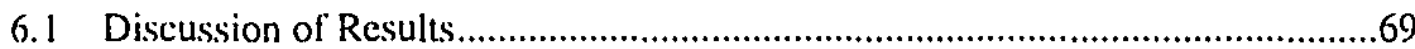

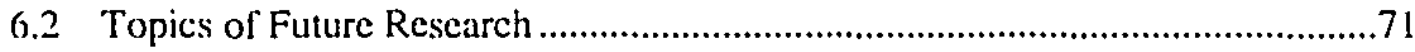

A Multitone Testing of the Log-Domain Filter...........................................................

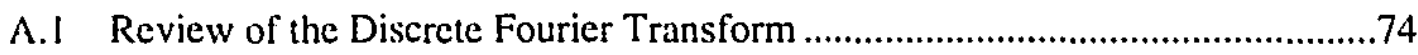

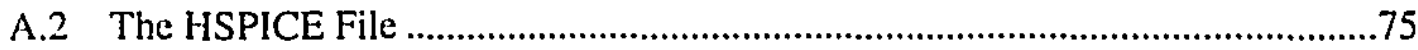

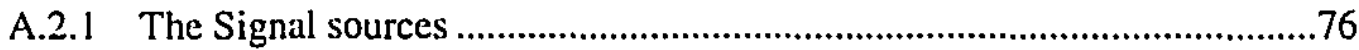

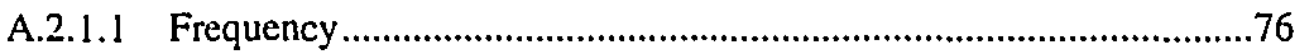

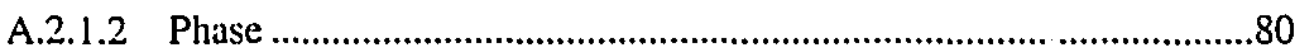

A.2.1.3 Amplitude..............................................................................80

A.2.1.4 A complete HSPICE current source ...............................................80

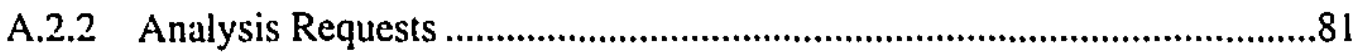

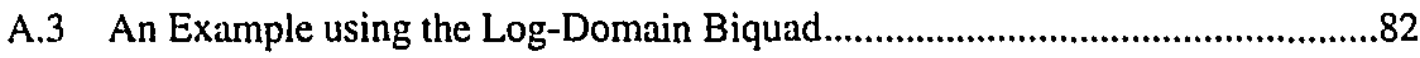

A.4 C Program for Generating the HSPICE File ..........................................................

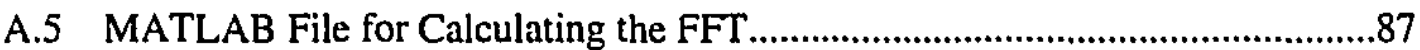

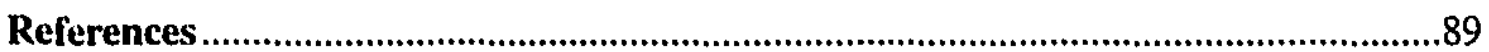




\section{List of Figures}

1-1 (a) MOS resistor (b) Single-ended MOS-C integrator (c) Fully-ballanced MOS-C integrator

1-2 (a) OTA symbol (b) Simple implementation of an OTA (c) Single-ended gm-C integrator (d) Balanced integrator ..........................................................................5

1-3 A simple log-domain integrator......................................................................

1-4 (a) LC ladder for a 3rd-order lowpass filter (b) Associated SFG (c) Active-RC Integrator (d) Complete active-RC circuit

2-1 The basic log-domain cell.................................................................................12

2-2 (a) Physical implementation of the ANTI-LOG function (b) Physical implementation of the LOG function

2-3 (a) A linear system (b) A corresponding log-domain system.................................15

2-4 Linearizing a log-domain system ……………........................................................17

2-5 Log-domain circuit which implements the multiple input non-inverting integrator 18

2-6 SFG of a log-domain multiple-input non-inverting integrator ..................................19

2-7 (a) Log-domain circuit for the multiple-input inverting integrator (b) Equivalent SFG.

2-8 SFG of a log-domain integrator with damping 
2-8 Two equivalent circuits for the damped log-domain integrator

2-9 SFG of a simple log-domain system with input and output .23

2-10 Circuit for the implementation of a simple system with input and output (b) Simplified circuit

3-1 (a) SFG of a typical LC ladder (b) Log-domain equivalent SFG ..........................28

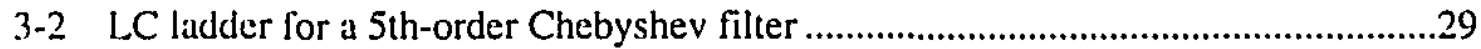

3-3 (a) SFG of a fifth-order Chebyshev filter (b) The log-domain equivalent system ...31

3-4 Circuit diagram for the fifth-order Chebyshev log filter 33

3-5 Multitone analysis of the fifth-order log-domain Chebyshev filter (a) Ideal txs (b) Gennum txs

3-6 AC analysis of the 5th-order Chebyshev log-domain filter (a) Full frequency (b) Passband

3-7 Tunability of the log-domain filter 38

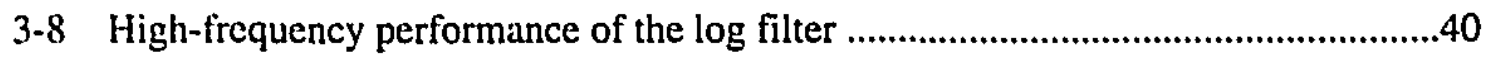

3-9 Intercept plots for the log-domain filter (a) Ideal txs (b) Gennum txs 41

3-10 Frequency plots for calculating the THD of the log-domain filter (a) Ideal txs (b) Gennum txs

3-11 Intermodulation distortion in the log-domain filter. .44

4-1 (a) LC ladder for a 3rd-order elliptic filter and its associated frequency response ..47

4-2 (a) SFG of a 3rd-order elliptic filter (b) The log-domain equivalent system ...........48

4-3 Circuit diagram for a 3rd-order elliptic log-domain filter 49.

4-4 Frequency analysis of the log-domain elliptic filter (a) Multitone analysis (b) AC analysis (full scale) (c) AC analysis (passband).

4-5 (a) Lowpass Prototype (b) Bandpass LC ladder (c) Magnitude response of the bandpass LC ladder.

4-6 (a) SFG for the 4th-order bandpass filter (b) Equivalent log-domain SFG. .53 
4-7 A 4th-order log-domain bandpass filter.

4-8 Simulated frequency response of the bandpass log-domain filter (a) Multitone analysis (b) AC analysis

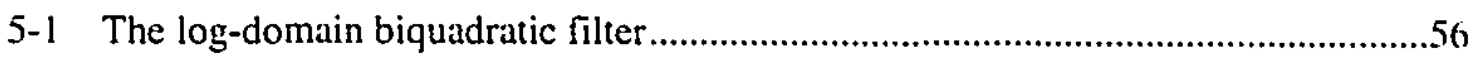

5-2 Microphotograph of the log-domain biquad.....................................................57

5-3 The complete test-circuit for the log-domain tilter .........................................5

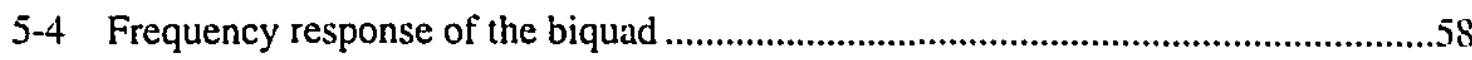

5-5 (a) Tunability of the log-domain biquad (b) The magnitude response of the biquad for 4 different cutoff frequencies .59

5-6 Spectral response of the biquad (a) Stimulated by a single tone (b) Stimulated by two

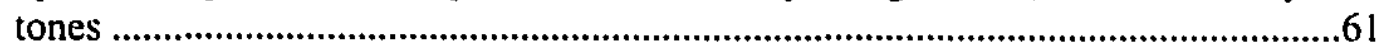

5-7 (a) IMD vs. input current (b) SNR vs. input current (over a $50 \mathrm{kHz} \mathrm{BW) \ldots \ldots \ldots \ldots ...62}$

5-8 (a) Magnitude response of the 5th-order Chebyshev log filter (b) Close-up of the passband

5-9 (a) Tunability of the 5th-order log filter (a) Full frequency range (b) Passband......64

5-10 (a) THD of the log-domain Chebyshev filter vs. input current (b) Spectral plot of the filter output for a single tone input.

5-11 (a) IMD of the Chebyshev log filter vs. input current (b) Spectral plot of the filter output for a two tone input.

5-12 Frequency response of the elliptic log-domain filter (a) Full frequency scale (b) Passband .68

A-1 Calculating the unit time period of a 3 tone input .76

A-2 Illustrating the relationship between the sampling frequency and other multitone parameters. .77

A-3 FFT response of the log-domain biquad .83 


\section{List of Tables}

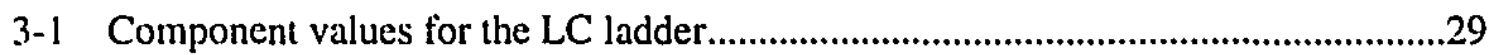

3-2 Component values for the 5th-order Chebyshev log-domain filter circuit................32

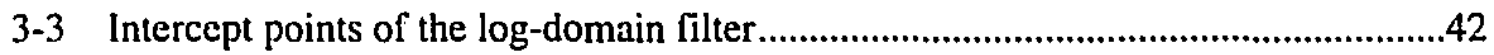

3-4 Total harmonic distortion in the log-domain filter ..............................................44

5-1 THD measurements for the log-domain biquad ...........................................60

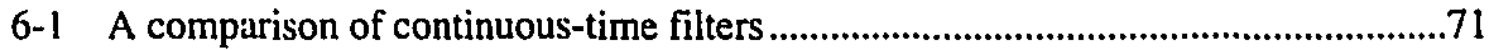


$\overline{\text { CHAPTER } 1}$ Introduction

\subsection{Motivation}

Frequency shaping networks, or filters, are key elements in many of today's microelectronic systems. They can be found in everything from cellular phones to data converters to home audio components. Filters generally fall into three broad categorics: continuous-time, sampled-data or fully digital. Digital filters have all of the advantages associated with digital systems and can be easily incorporated inside the DSP core of an integrated circuit. They are best suited for lower frequency applications and find widespread use because of the increasingly popularity of fully digical design. Sampleddata filters combine analog filtering techniques with digital sampling principles. This makes them ideally suited for data converters (analog-to-digital or digital-to-analog) which must interface between the real analog world and the digital core of most microelectronics. Sampled-data systems generally use MOS technology which allows them to be readily integrated on the same chip as the digital circuitry. Continuous-time filters make up a small but important part of the filter design area. They are especially effective when dealing with real-world (analog) signals and are commonly used in highfrequency, low-power systems. This last advantage makes them a popular choice for the 
growing wireless industry.

The two most popular forms of integrated continuous-time filters are transconductance-C $\left(\mathrm{g}_{\mathrm{m}}-\mathrm{C}\right)$ filters and MOS-C filters [1]. These two filter types are well suited for integrated circuits as they lend themselves well to fully differential design and can be fabricated using only grounded capacitors. MOS-C filters show good distortion behavior but suffer at high speeds due to the frequency compensation of the op-amps. Transconductance-C filters offer greater bandwidth but this often comes at the expense of linearity.

One filtering method which has recently proposed and which has the potential to combine high-frequency performance with low distortion levels is the log-domain filter. Log-domain filters contain low impedance nodes along the signal path which they exploit to achieve greater bandwidths. In addition, they are a form of translinear circuit [2] thus they make use of the exponential nature of a bipolar transistor and do not require that the transistor be linearized. These properties along with the fact that they have current input and output has led them to be classified as "current-mode" circuits [3].

Although the concept of log-domain filtering has been around since the 1970 s [4], it has only recently been expanded into a general filter design approach [5]. This design method proposed by Frey is based on a state-space approach and on a set of mathematical mappings which replace the existing state-space variables by a related exponential function. Not only is this method complicated but the set of equations which ends up representing the filter offers very little insight into its operation. This is particularly troublesome when dealing with a novel and unconventional filtering scheme like the one proposed herein.

This thesis will propose a complete method of design for log-domain filters which is based on the operational simulation of LC ladders [6]. By doing so, we benefit not only from the wealth of knowledge which has been accumulated over the years on 
these circuits and on their many properties but, more importantly, we benefit from the basic insight which comes from using ladders as a basis for filter design. By establishing at set of blocks which can be used to implement the LC ladder, the designer can tell at a glance which parts of the circuit give rise to the different poles and zeros. This should be of great help for both understanding these circuits and for investigating their limitations.

\subsection{Conventional Integrated Continuous-Time Filters}

\subsubsection{MOS-C Filters}

A MOS-C filter can be constructed by replacing the resistor in a standard active$\mathrm{RC}$ filter by a MOS transistor biased in the triode region [ 1 ]. The equation for the current flowing through such a MOSFET (shown in Figure 1-1(a)) would be [7]:

$$
i_{D}=\frac{1}{2} \mu C_{O X}\left(\frac{W}{L}\right)\left[2\left(v_{C}-v_{2}-V_{T}\right)\left(v_{1}-v_{2}\right)-\left(v_{1}-v_{2}\right)^{2}\right]
$$

Eq. (1.1) can be separated into linear and non-linear terms, such that:

$$
i_{D}=\mu C_{O X}\left(\frac{W}{L}\right)\left(V_{C}-V_{T}\right)\left(v_{1}-v_{2}\right)+\text { a non-linear term }
$$

Therefore, if we neglect the non-linear part of the equation, the MOSFET can be used as a voltage-controlled resistor where:

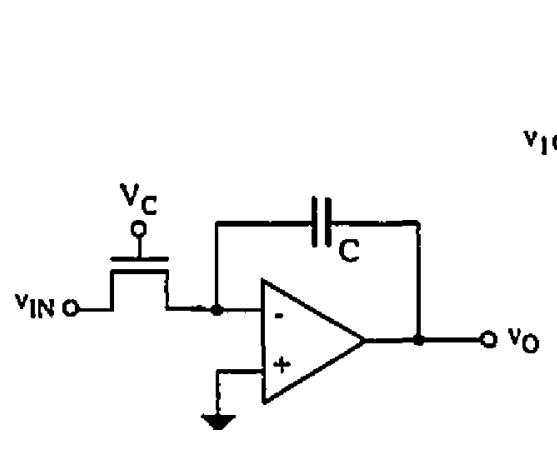

(b)

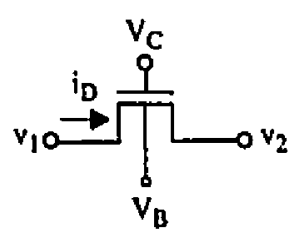

(a)

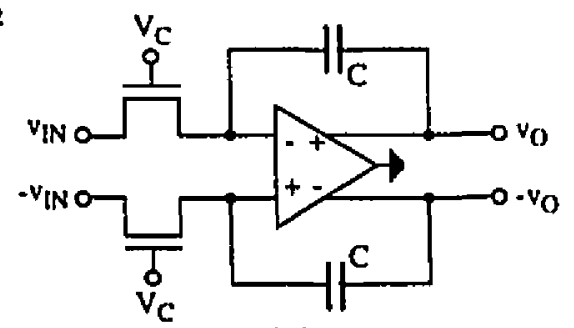

(c)

Figure 1-1: (a) MOS resistor (b) Single-ended MOS-C integrator (c) Fully-balanced MOS-C integrator 


$$
R\left(V_{C}\right)=\frac{1}{\mu C_{O X}\left(\frac{W}{L}\right)\left(V_{C}-V_{T}\right)}
$$

Such an approach would result in the single-ended MOS-C integrator shown in Figure 1l(b). Unfortunately, the non-linear portion of the MOS current equation will be appreciable for all but the smallest input levels. One solution is to use a fully-balanced design like the one shown in Figure 1-1(c). This eliminates the even portion of the nonlinear function which accounts for most of the non-linearity (the odd portion typically accounts for less than $0.1 \%$ or $-60 \mathrm{~dB}$ ).

The design of different types of MOS-C filters would follow the same approach as is used for the design of active-RC filters [6]. This would typically involve a technique like the operational simulation of LC ladders used in this thesis. Because of the voltage controlled resistor, these types of filters are well suited to designs which incorporate some form of automatic tuning [8-10]. MOS-C filters typically show distortion levels in the order of $40-60 \mathrm{~dB}$ due to the non-linearity of the MOS transistor as a resistor, however with feedback techniques, distortion levels of $-90 \mathrm{~dB}$ have been reached [11]. Their biggest drawback is that they are limited in bandwidth by the op-amps and thus are not particularly well suited to high-frequency applications.

\subsubsection{Transconductance- $\mathrm{C}\left(\mathrm{g}_{\mathrm{m}}-\mathrm{C}\right)$ Filters}

Transconductance-C filters are based on the operational transconductance amplifier (OTA) which, as the name implies, generates an output current which is proportional to the input current by a factor of $\mathrm{g}_{\mathfrak{m}}$. The symbolic representation for an OTA is shown in Figure 1-2(a) along with a simple MOS implementation which is composed of a differential pair, 3 current mirrors and a simple current source (Figure 12(b)) [12]. The OTA can be used to build an integrator by simply pushing the output current into a capacitor as is shown in Figure 1-2(c). Writing an equation for the transfer function, $\mathrm{H}(\mathrm{s})$, of the gm-C integrator, we get: 


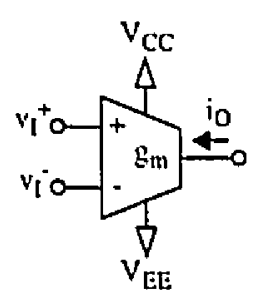

(a)

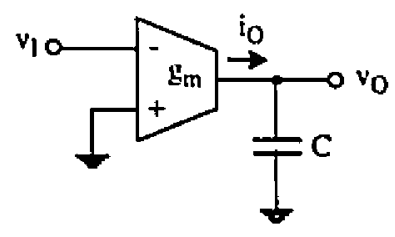

(c)

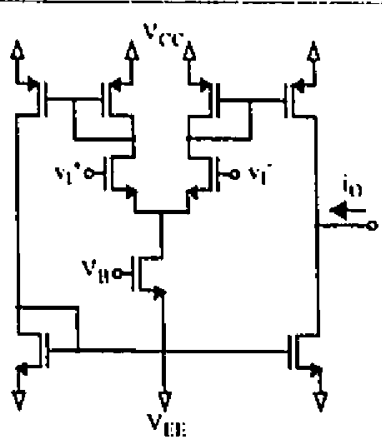

(b)

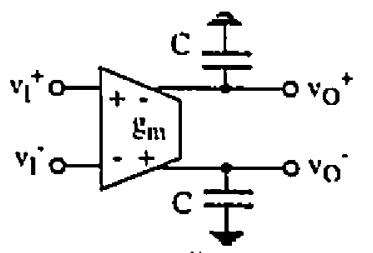

(d)

Figure 1-2: (a) OTA symbol (b) Simple implementation of an OTA

(c) Single-ended $\mathrm{g}_{\mathrm{m}}$-C integrator (d) Balanced integrator

$$
H(s)=\frac{v_{O}(s)}{v_{l}(s)}=\frac{g_{m} v_{l}\left(\frac{1}{C s}\right)}{v_{l}}=\frac{1}{s\left(C / g_{m}\right)}
$$

In order to increase the signal-to-noise ratio by reducing the common-mode noise caused by things like the digital circuit switching transients, these filters are typically built using a fully differential, balanced form like the circuit shown in Figure 1-2(d). Given the $\mathrm{g}_{\mathrm{m}}-\mathrm{C}$ integrator, traditional filter design methods such as $\mathrm{LC}$ ladder simulation can be used to achieve different filter configurations.

Transconductance-C filters are more suited to high-speed applications than the MOS-C filters described previously since they can be used in an open-loop configuration and thus need not be constrained by the stability requirements which limit op-umps. Several $g_{m}-C$ filter designs have recently been proposed which are suitable for video-rate applications $[13,14]$. The drawback to using the OTA in an open-loop configuration is that the circuit is limited to very small input levels in order to keep it relatively linear. For 
example, the circuit of Figure 1-2(b) would need a differential input of less than $50 \mathrm{mV}$ for reasonable results. Many different techniques have been proposed which increase the input range while maintaining linearity but these often degrade the frequency response due to added parasitics [15]. Several circuits which combine low distortion with a relatively high bandwidth can be found in the literature [16,17]. A final drawback

concerning $\mathrm{g}_{\mathfrak{m}}-\mathrm{C}$ filters is their dependence on the parameter $\mathrm{g}_{\mathrm{m}}$ which makes them highly susceptible to process variations. This can be accounted for on-chip by including some form of automatic tuning $[18,19]$.

Although we have focused on MOS-C and $g_{m}-C$ filters there exists a variety of different continuous-time integrated filter design methods in the literature. They resemble one another only in the fact that they share some common design goals, namely tunability, linearity and high-frequency operation. Often, optimization of a filter in terms of one or two of these characteristics comes at the expense of the others. The log-domain filter is unique in that it has the potential to achieve good performance in all three of these areas.

\subsection{A Novel Approach to Continuous-Time Filtering: The Log-Domain Filter}

The concept of log-domain filtering was first introduced by Adams in 1979 [4]. He recognized that the diode-capacitor combination shown in Figure 1-3 could be used as a "log-domain" integrator. The reasoning can be summarized as follows. Let us assume that the current flowing through the diode of Figure 1-3 is given by a simple exponential function. The circuit could hence be described by the equation:

$$
e^{\left(v_{1}-v_{o}\right)}=C \frac{d}{d t}\left(v_{0}\right)
$$

Which can be rewritten as:

$$
e^{v_{1}}=C \frac{d}{d t}\left[e^{v_{0}}\right]
$$




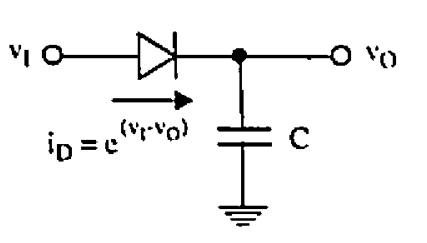

Figure 1-3: A simple log-domain integrator

If the two exponential functions of $\mathrm{Eq}$. (1.6) are replaced by simple variables, i.c.

$$
x_{l} \Leftrightarrow e^{\nu_{I}} \& x_{O} \Leftrightarrow e^{"_{0}}
$$

then a linear differentiation (or integration) operation is performed. Additional exponential circuits can be used to establish these relationships. Adams derived a number of different filter circuits based on this premise. One characteristic of these filters which makes them easily tunable is that their cutoff frequency is dependent on the bias current.

This concept remained largely unexplored until recently when Frey introduced a much more generalized approach to the design of log-domain filters [5]. Frey recognized that these filters possessed many of the same properties as current-mode circuits. His work presented a design method which was based on a state-space approach and which involved replacing the different variables in the state-space equations by related exponential functions. Bipolar circuits were then used to implement the set of equations. Using this approach he showed the design of a biquad and of a seventh-order Chebyshev filter formed by a cascade of biquads. Frey has published additional work in this area including a log-domain filter design which is formed entirely of NPN transistors and thus is suitable for very high-speed applications [20].

\subsection{The Operational Simulation of $L C$ Ladders}

The simulation of LC ladders finds widespread use as a filter design method for two major reasons [6]. The first reason is due to the straightforward relationship between the transmission zeros of the ladder transfer function and the different impedances in the 
ladder arms. This means that the filter designer can tell at a glance which elements give rise to the different transmission zeros thus making the task of tuning and debugging these circuits much easier. The second advantage comes from the excellent sensitivity properties exhibited by lossless, doubly-terminated ladders [21,22]. In simple terms, the highly coupled nature of the circuit makes the voltage (current) at any one node dependent on the values at all the other nodes. This serves to distribute the effect of process variations over the entire circuit and makes the circuit less sensitive to individual variations.

Unfortunately, the passive LC ladder is of limited use since inductors do not lend themselves well to integrated circuit design. As a result, many different active circuit implementations of the LC ladder have been invented including the two discussed in the Section 1.2. The design of active filter circuits based on the simulation of LC ladders can be further broken down into two different methods: component simulation and operational simulation. Component simulation simply involves replacing the inductors in the LC ladder by active circuits which perform the same operation. Some popular choices are the Antoniou general impedance converter (GIC) and circuits based on the Bruton transformation [6].

The operational simulation of LC ladders involves finding an active circuit which will mimic all of the voltage and current relationships within the LC ladder. The designer first writes a set of equations which completely specifies the operation of the ladder then draws a graphical representation of these equations called the signal-flow-graph (SFG). The signal-flow-graph is generally composed of integrators, summers and multipliers. Once the signal-fiow graph has been established it can be implemented using any of a number of methods including active-RC, MOS-C and $g_{m}-C$.

This concept is best illustrated through an example. We will show the design of a third-order active-RC lowpass filter based on this approach. First, a ladder which meets a desired set of specifications is found. This can be done through hand analysis [6], by using 

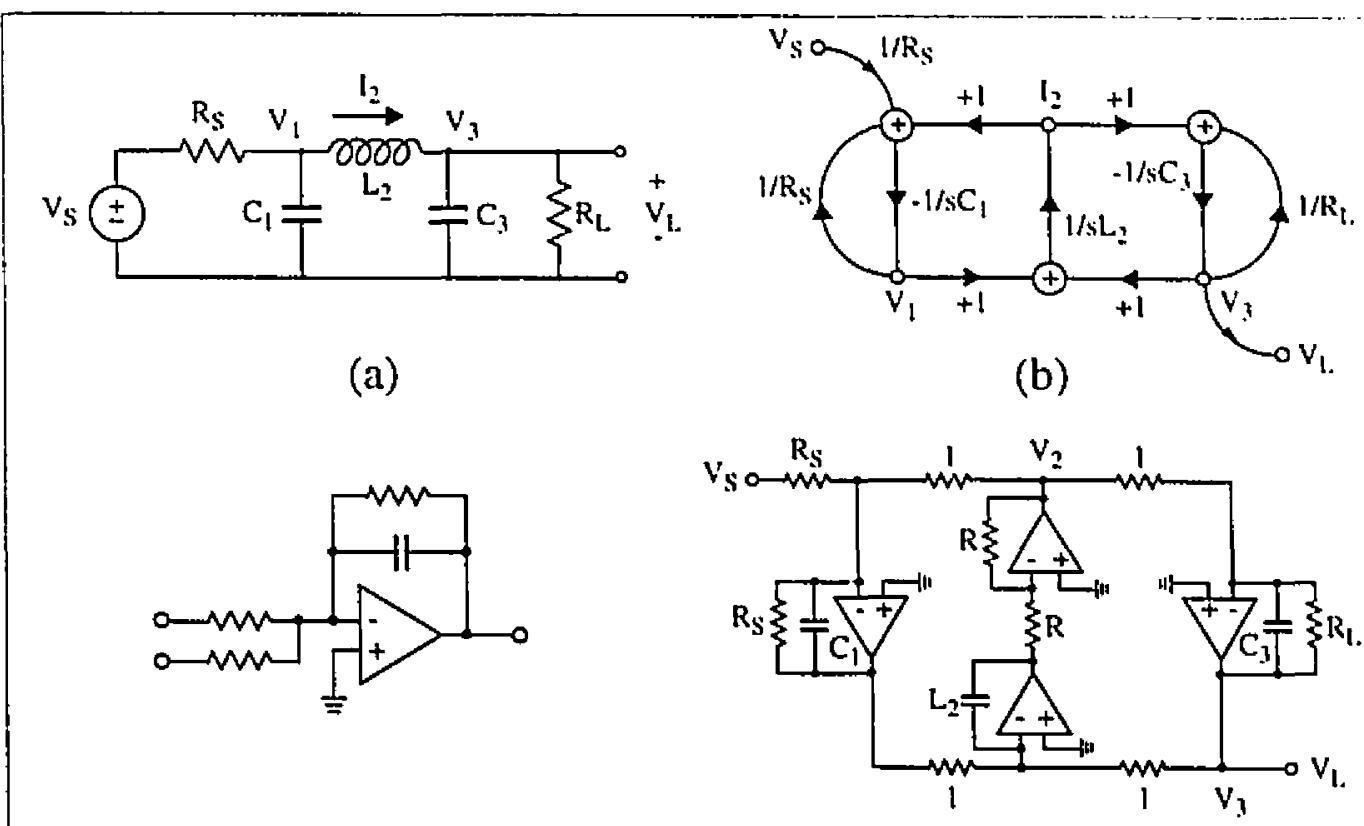

(c)

(d)

Figure 1-4: (a) LC ladder for a 3rd-order lowpass filter (b) Associated SFG (c) Active-RC Integrator (d) Complete active-RC circuit

look-up tables $[6,23]$ or by using a filter design program $[24,25]$. The LC ladder for the third-order lowpass filter is shown in Figure 1-4(a). Next a set of equations is derived which represents the LC ladder and a signal-flow graph is drawn (Figure 1-4(b)). The complete derivation of a signal-flow graph based on the relationships in an LC ladder can be found in Section 3.3.2. The final step is to inplement the signal-flow graph using active-RC blocks like the damped integrator shown in Figure 1-4(c). The complete circuit is shown in Figure 1-4(d).

\subsection{Thesis Outline}

This thesis will present a novel method for the design of log-domain filters which is based on the operational simulation of LC ladders.

Chapter 2 will introduce a set of log-domain building blocks which can be used to implement the signal-flow graph of an LC ladder. Because of the non-linear nature of 
the log-domain integrator a unique linearization procedure is proposed.

Chapter 3 shows how to use the building blocks introduced in Chapter 2 to design arbitrary filters based on the operational simulation of LC ladders. The method is illustrated through the design of a fifth-order Chebyshev filter. The resultant filter is simulated using HSPICE analysis. Unfortunately, AC analysis cannot be used since it relies on linearizirig the transistors and thus negates the exponential nature of the bipolar transistor. Instead, a technique called multitone analysis is used which is based on HSPICE transient analysis. The simulation results will focus on two areas, frequency response and linearity, since these best allow us to judge the usefulness of the new technique.

Chapter 4 extends these ideas to the design of filters with finite transmission zeros, namely elliptic and bandpass filters. A third-order elliptic filter and a fourth-order bandpass filter are designed. The implementation of the elliptic filter using the available building blocks will require an approximation which is described in this chapter. Performance of both filters will be verified through HSPICE analysis.

Chapter 5 presents experimental results which verify the ideas presented in the previous chapters. These results are of particular importance since they represent the first reported experimental data on the log-domain filter. The chapter contains results from experiments performed on a log-domain biquad, a fifth-order Chebyshev filter and a fifthorder elliptic filter. The frequency response of all filters is plotted versus expected and simulated results. Their tunability and high-frequency properties are examined. Distortion levels are found by measuring total harmonic distortion, intermodulation distortion and signal-to-noise ratio.

The final chapter evaluates the performance of these filters in comparison with other continuous-time filtering techniques and discusses some areas of future work. 


\section{$\overline{\text { CHAPTER } 2}$ The Basic Log-Domain Building}

\section{Blocks}

As described in the introduction, the operational simulation of LC ladders is based on replacing the different elements of a signal-flow graph by the appropriate circuits. For example in the active-RC example given previously, the elements of the signal-flow graph were replaced by op-amp based integrators. The main operations which need to be performed are addition, multiplication and integration. This chapler will describe the log-domain circuits which provide these functions.

Because of the unique nature of log-domain circuits, we first give a symbolic description of the log-domain building blocks. We will show how these blocks are nonlinear in nature but that they can be interconnected in such a way as to give a linear inputoutput relationship. The reader is introduced to the basic log-domain cell, a simple circuit which will figure prominently in the rest of the thesis. Two mathematical operators will be introduced and then used to establish a correspondence between traditional linear systems and the log-domain systems. Once this background has been established, a library of basic log-domain circuits will be presented. 


\subsection{The Log-Domain Cell}

We begin our journey into the world of log-domain filters with the analysis of a simple circuit which will prove to be the comerstone of any filter constructed using this approach. This simple translinear circuit will be referred to as the basic log-domain cell and is shown in Figure 2-1.

Analysis of this circuit can be performed as follows. Applying KVL around loop - and neglecting base currents, we can write:

$$
-V_{A}-V_{E B_{1}}-V_{B E_{2}}+V_{B E_{3}}+V_{E B_{4}}+V_{B}=0
$$

For calse of understanding, the following emitter voltages are defined:

$$
\begin{aligned}
& V_{B E_{\mathrm{A}}}=V_{E B_{1}}+V_{B E_{2}} \\
& V_{B E_{B}}=V_{B E_{3}}+V_{E B_{4}}
\end{aligned}
$$

The voltages $V_{B E_{A}}$ and $V_{B E_{B}}$ can be related to the currents $K \cdot I_{0}$ and $I_{B}$ according to the bipolar transistor equation (the Early effect has been neglected). This results in:

$$
K \cdot I_{o}=I_{s} \cdot e^{V_{B E_{A}} / 2 V_{T}}
$$

and,

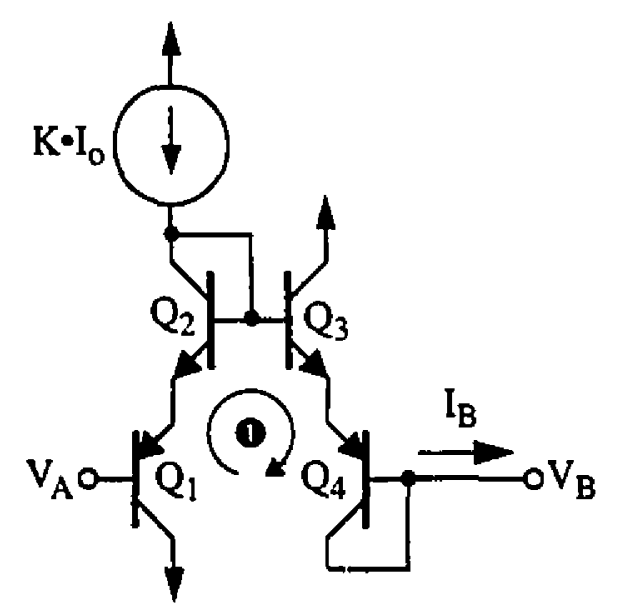

Figure 2-1: The basic log-domain cell 


$$
I_{B}=I_{s} \cdot e^{V_{A E_{B}} / 2 V_{T}}
$$

We now rewrite Eq. (2.1) as follows:

$$
V_{A}+V_{B E_{A}}-V_{B E_{B}}=V_{B}
$$

Replacing Eqs. (2.4) and (2.5) into Eq. (2.6) leads to:

$$
V_{A}+2 V_{T}^{\prime} \cdot \ln \left[\frac{K \cdot I_{0}}{I_{s}}\right]-2 V_{T} \cdot \ln \left[\frac{I_{B}}{I_{s}}\right]=V_{B}
$$

Simplifying and solving for $I_{B}$ gives:

$$
I_{B}=K \cdot I_{o} \cdot e^{\left(v_{A}-v_{B}\right) / 2 v_{T}}
$$

Eq. (2.8) is the basic log-domain equation and will be referenced throughout this thesis.

\subsection{The $L O G$ and $A N T I-L O G$ Operators}

Let us now define two new mathematical inverse operators, LOG and ANTILOG. These functions imitate the traditional logarithmic and exponential functions except that they can be implemented physically using the basic log-domain cell described in the previous section. Note that the purpose here is to define a new exponential function which is better matched to the physical circuits with which we will be dealing.

The ANTI-LOG function is essentially three mathematical steps in one:

1. Divide by $2 V_{T}$

2. Raise to the exponential

3. Multiply by $I_{o}$

Therefore it can be written as:

$$
\operatorname{ANTILOG}(X)=I_{o} \cdot e^{X / 2 V_{T}}
$$

This can be implemented by Eq. (2.8) if $K$ is equal to 1 and $V_{B}$ is tied to ground, i.c., 


$$
I_{B}=I_{o} \cdot e^{V_{\wedge} / 2 V_{T}}
$$

In other words, the ANTI-LOG function can be physically represented by the current flowing from a log-domain cell driven by a voltage $X$, as shown in Figure 2-2(a).

The LOG function is defined as the inverse of the ANTI-LOG function, such that:

$$
\operatorname{LOG}[\operatorname{ANTILOG}(V)]=V
$$

As a result, $L O G$ can be described mathematically by:

$$
\operatorname{LOG}(Y)=2 V_{T} \cdot \ln \left(\frac{Y}{I_{0}}\right)
$$

Again we see that this can be physically implemented by the log-domain cell, as shown in Figure 2-2(b).

We will now use these two operators to show the correspondence between a traditional linear system and the log-domain systems which we deal with in this thesis.

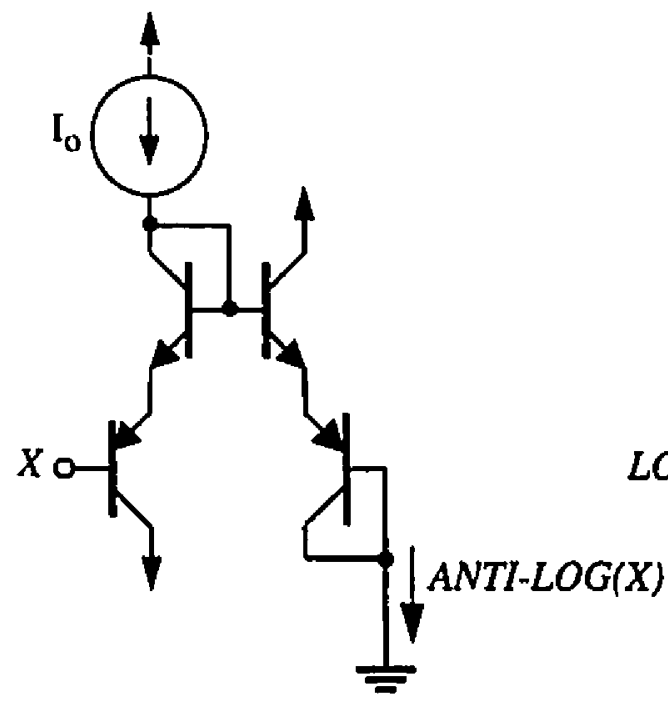

(a)

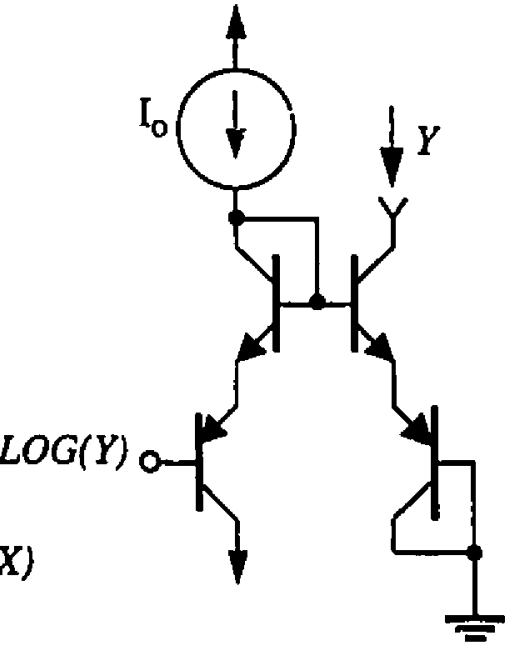

(b)

Figure 2-2: (a) Physical implementation of the ANTI-LOG function (b) Physical implementation of the $L O G$ function 
Chapter 2: The Basic Log-Domotin Building Blocks

\subsection{Characterizing a Log-Domain System}

The block dingram of a typical linear system is shown in Figure 2-3(i). In the context of filter design, such a system could represent anything from a simple integrator to an Nth-order LC ladder. This linear system can be transformed into a log-domain sysisem by placing ANTI-LOG blocks at the inputs and LOG block at the outpus. This is illustrated in Figure 2-3(b). We will see that all of the log-domain building blocks described in this section have this form. At this point, we have introduced some notation which will remain consistent throughout this work. Variables marked with the circumllex $\left({ }^{\wedge}\right)$ represent signals in the log-domain. This allows us to quickly distinguish between signals in the log-domain and signals in the traditional linear domain.

Let us examine the new system (Figure 2-3(b)) a little more closely. Assume that the linear system of Figure 2-3(a) is described by the equation:

$$
X_{o}=F\left(X_{i}\right)
$$

where $F$ is a linear function.

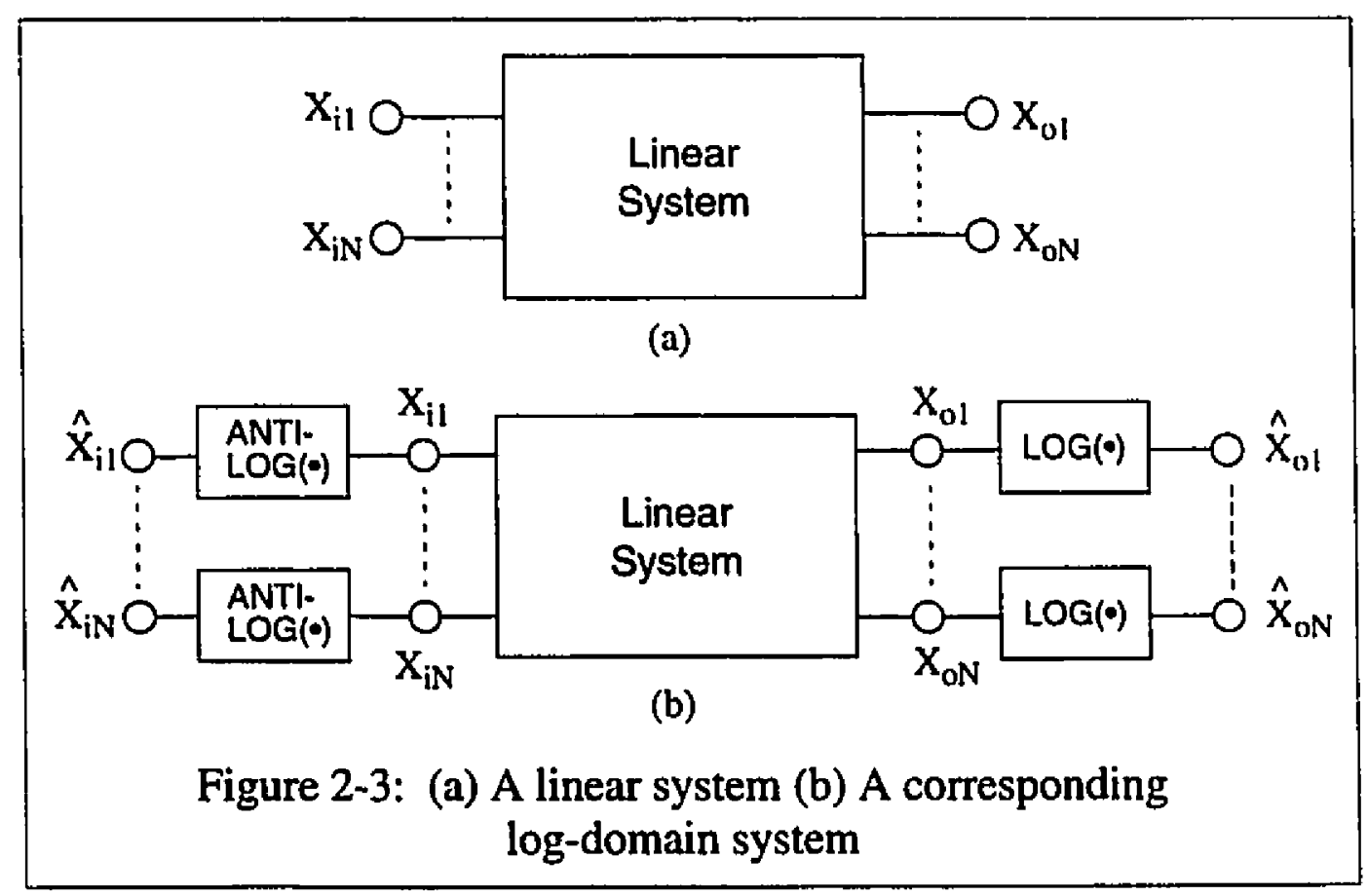


The new log-domain system would therefore be described by:

$$
\hat{X}_{o}=\operatorname{LOG}\left\{F\left[A N T I L O G\left(\hat{X}_{i}\right)\right]\right\}
$$

If we were to replace the $L O G$ and $A N T I-L O G$ by their original mathematical definitions then we would have:

$$
\hat{X}_{o}=2 V_{T} \cdot \ln \left\{F\left[I_{o} \cdot e^{\dot{X}_{i} / 2 V_{T}}\right]\right\} \neq F\left(\hat{X}_{i}\right)
$$

Even though the original function $F$ was linear, the nature of the logarithm function will result in $\hat{X}_{o}$ being a non-linear function of $\hat{X}_{i}$. Therefore we need to find a way to linearize the log-domain system and regain the original transfer function.

Consider placing $L O G$ blocks preceding the input and ANTI-LOG blocks after the output, as shown in Figure 2-4(a). Because of the inverse nature of these functions (see Eq. (2.11)), the overall result is a linear input-output relationship.

A second way to get the non-linearities to cancel is to simply join different logdomain sections together, as shown in Figure 2-4(b). This natural cancellation is what makes these circuits so powerful. Suppose that we can build log-domain circuits of the form shown in Figure 2-3(b) which perform the basic functions of summation, integration and multiplication. Then we would simply need to join the different blocks together in the required loops, add the inverse function at the input and the output, and we would get the desired linear transfer function. The next section will show a library of log-domain circuits which have exactly this form. 


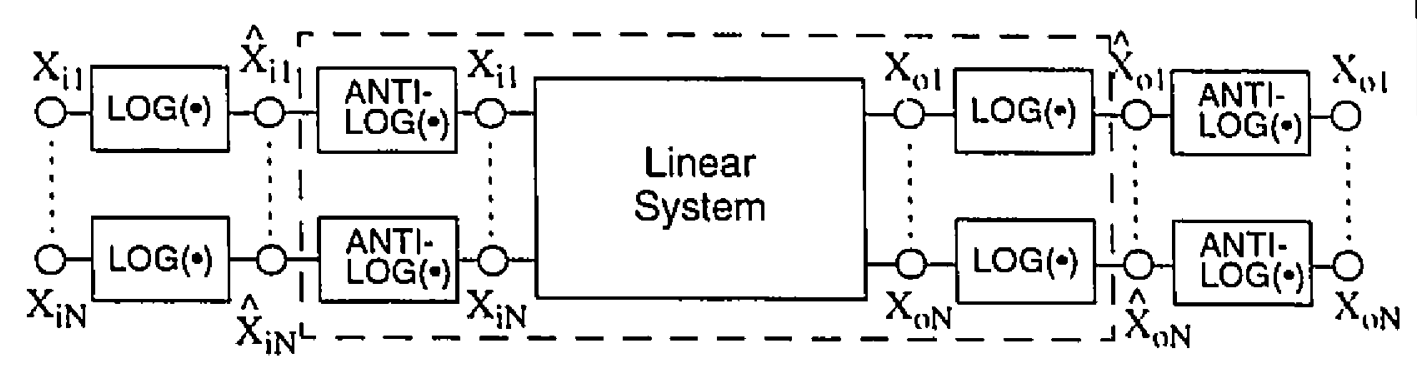

(a)

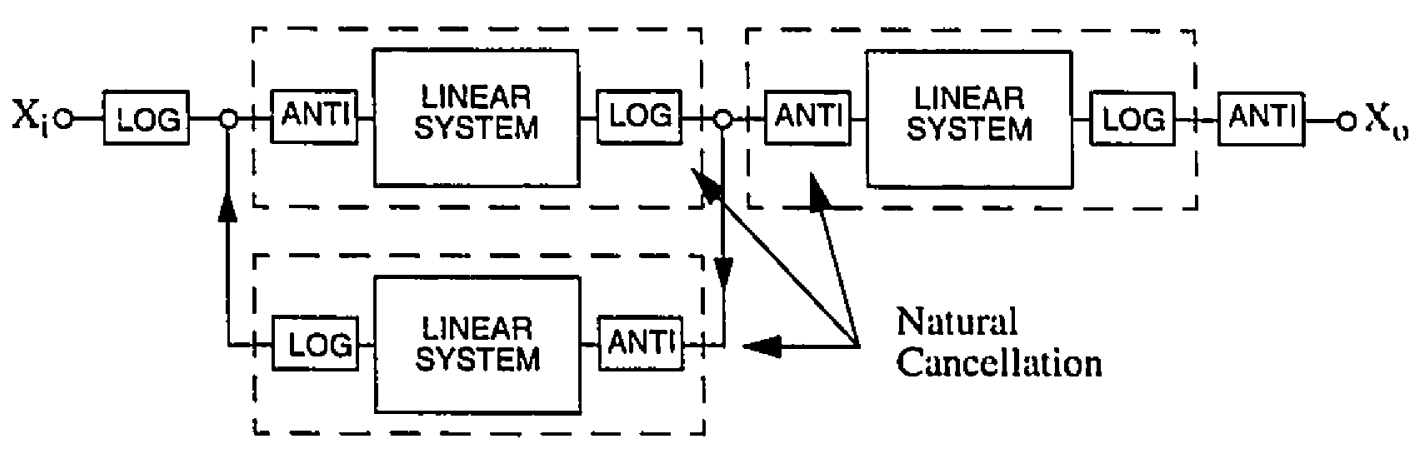

(b)

Figure 2-4: Linearizing a log-domain system

\subsection{The Basic Building Blocks}

This section will introduce the different blocks which perform the summing, integration and multiplication operations necessary for log-domain filter design. For each different operation, we will first introduce the circuit which performs the desired task. Then, basic circuit analysis will be used to show how they give the required log-domain function. We pay particular attention to the input and output sections since these play an important role in linearizing the overall system.

\subsubsection{The Non-Inverting Integrator}

The concept of a log-domain integrator was first introduced by Adams [4]. More recently Frey has proposed a bipolar version [5] which has led to the multiple-input non- 


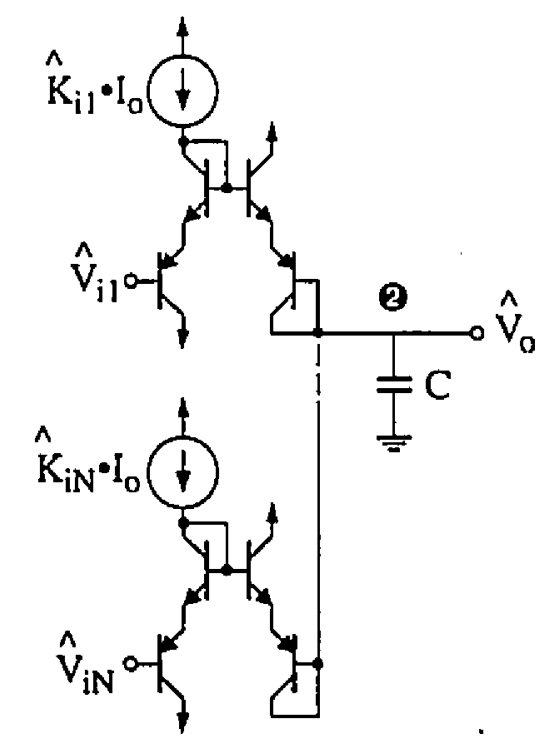

Figure 2-5: Log-domain circuit which implements the multiple input non-inverting integrator

inverting integrator shown in Figure 2-5. Analysis of the circuit can be described as follows.

Using the basic log-domain equation (Eq. (2.8)) and applying $\mathrm{KCL}$ at node 2 allows us to write the equation:

$$
\hat{C} \cdot \frac{d}{d t} \hat{V}_{o}=\hat{K}_{i_{1}} \cdot I_{o} \cdot e^{\left(\hat{v}_{i_{1}}-\hat{v}_{0}\right) / 2 v_{T}}+\ldots+\hat{K}_{i_{N}} \cdot I_{o} \cdot e^{\left(\hat{v}_{i_{N}}-\hat{v}_{0}\right) / 2 V_{T}}
$$

Multiplying through by $e^{\hat{v}_{i \prime} / 2 V_{T}}$ leads to:

$$
\hat{C} \cdot e^{\hat{V}_{0} / 2 V_{T}} \cdot \frac{d}{d t} \hat{V}_{0}=\hat{K}_{i_{1}} \cdot I_{0} \cdot e^{\hat{V}_{i_{1}} / 2 V_{T}}+\ldots+\hat{K}_{i_{N}} \cdot I_{0} \cdot e^{\hat{V}_{i_{N}} / 2 V_{T}}
$$

Using the chain rule to rewrite the derivative gives:

$$
\frac{\hat{C} \cdot 2 V_{T}}{I_{o}} \cdot \frac{d}{d t}\left[I_{0} \cdot e^{\hat{V}_{i} / 2 V_{T}}\right]=\hat{K}_{i_{1}} \cdot I_{o} \cdot e^{\hat{V}_{i_{1}} / 2 V_{T}}+\ldots+\hat{K}_{i_{N}} \cdot I_{0} \cdot e^{\hat{V_{i_{N}} / 2 V_{T}}}
$$

The factor $l_{o} / 2 V_{T}$ can be incorporated into a new constant which will be denoted simply 
as $K_{i}$, such that:

$$
K_{i}=\frac{\hat{K}_{i} \cdot I_{o}}{2 V_{T}}
$$

This allows us to rewrite Eq. (2.18) in a form which will be easier to relate to the signalflow graph of an LC ladder,

$$
C \cdot \frac{d}{d t}\left[I_{0} \cdot e^{\hat{v_{o} / 2 V_{T}}}\right]=K_{i_{1}} \cdot I_{0} \cdot e^{{\hat{V_{i}}}_{\mathrm{i}} / 2 V_{r}}+\ldots+K_{i_{N}} \cdot I_{0} \cdot e^{\hat{v_{i_{N}} / 2 V_{r}}}
$$

The factor $I_{0}, 2 V_{T}$ will be important when we are transforming the LC ladder into a logdomain circuit. It can either be incorporated into the multiplication factors $K_{i}$ and thus will affect the bias current or else it can be used to scale the capacitors as will be shown in the next chapter. It is this factor which accounts for the good tunability properties of logdomain filters. Note that in addition to making the filter dependent on the bias currents in the circuit, the factor $I_{o} / 2 V_{T}$ also makes the circuit temperature dependent ${ }^{\dagger}$.

Returning to the analysis of the non-inverting integrator, Eq. (2.20) can be rewritten using the $A N T I-L O G$ operator, such that:

$$
C \cdot \frac{d}{d t}\left[\operatorname{ANTILOG}\left(\hat{v}_{o}\right)\right]=K_{i_{1}} \cdot \operatorname{ANTILOG}\left(\hat{V}_{i_{1}}\right)+\ldots+K_{i_{N}} \cdot \operatorname{ANTILOG}\left(\hat{V}_{i_{N}}\right)
$$

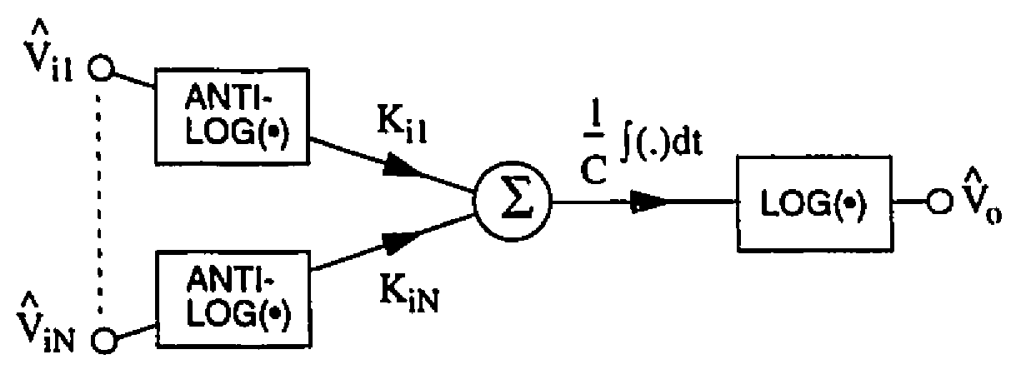

Figure 2-6: SFG of a log-domain multiple-input non-inverting integrator

$t$. The fact that we know that the behavior of the circuit is directly proportional to $V_{T}$ and hence to temperature makes it relatively easy to account for. This can be done by using a temperature compensation scheme as is done in temperature independent biasing [12]. 
For the final form of the equation, $\hat{V}_{o}$ is isolated, giving:

$$
\hat{V}_{0}=\operatorname{LOG}\left\{\frac{1}{C} \cdot \int\left[K_{i_{1}} \cdot A N T I L O G\left(\hat{V}_{i_{1}}\right)+\ldots+K_{i_{N}} \cdot \operatorname{ANTILOG}\left(\hat{V}_{i_{N}}\right)\right] d t\right\}
$$

The signal-flow graph of this system is shown in Figure 2-6. Clearly, this circuit has the desired log-domain form; it contains a linear system at the centre with ANTI-LOG and $L O G$ blocks at the input and output respectively. The only difference between this system and the ones we defined in Section 2.3 is that the inputs and outputs are now given as voltages as opposed to a general variable. This is due to the physical circuit which was used to implement the system.

\subsubsection{The Inverting Integrator}

We now present the multiple-input inverting integrator whose circuit is shown in Figure 2-7(a). This circuit can be analyzed in the exact same manner as for the noninverting integrator. The first step is to find the sum of the currents at node 3, giving:

$$
\hat{C} \cdot \frac{d}{d t} \hat{V}_{o}=-\hat{K}_{i_{1}} \cdot I_{o} \cdot e^{\left(\hat{V}_{i_{1}}-\hat{V}_{o}\right) / 2 V_{T}}-\ldots-\hat{K}_{i_{N}} \cdot I_{o} \cdot e^{\left(\hat{V}_{t_{N}}-\hat{V}_{0}\right) / 2 V_{T}}
$$

Comparing Eq. (2.23) to Eq. (2.16) shows that the analysis which was performed in Section 2.4 .1 can now be repeated simply by replacing the coefficients $K_{i l}, K_{i 2}, \ldots, K_{i N}$ by their negative counterparts. Knowing this, $\hat{V}_{o}$ can now be written as

$$
\hat{V}_{o}=\operatorname{LOG}\left\{\frac{1}{C} \int\left[\left(-K_{i_{1}}\right) \cdot A N T I L O G\left(\hat{V}_{i_{1}}\right)+\ldots+\left(-K_{i_{N}}\right) \cdot \operatorname{ANTILOG}\left(\hat{V}_{i_{N}}\right)\right] d t\right\}(2.24)
$$

Taking the negative sign outside of the integral gives:

$$
\hat{v}_{o}=\operatorname{LOG}\left\{-\frac{1}{C} \cdot \int\left[K_{i_{1}} \cdot \operatorname{ANTILOG}\left(\hat{V}_{i_{1}}\right)+\ldots+K_{i_{N}} \cdot \operatorname{ANTILOG}\left(\hat{V}_{i_{N}}\right)\right] d t\right\}
$$

This results in the signal-flow graph shown in Figure 2-7(b). Again we see the familiar pattern representative of $\log$-domain systems. 


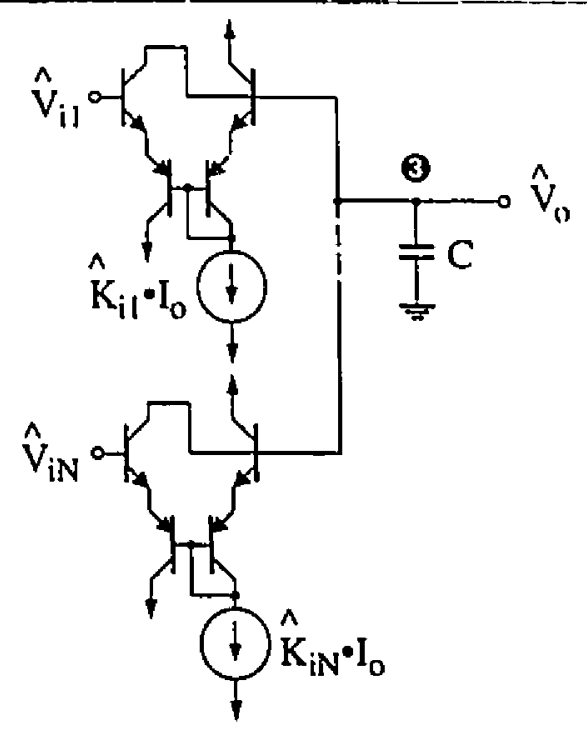

(a)

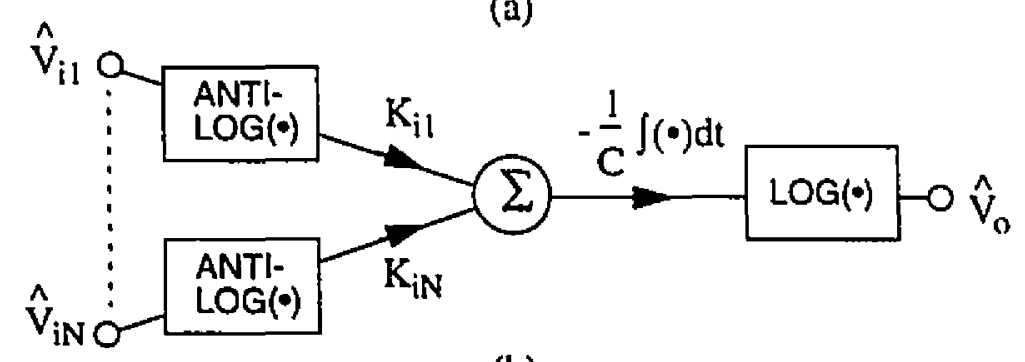

(b)

Figure 2-7: (a) Log-domain circuit for the multiple-input inverting integrator (b) Equivalent SFG

\subsubsection{Damping}

The damped integrator is simply an extension of the multiple-input integrator shown previously. It is presented here separately due to its importance as a building block in filter circuits and because of an important simplification which arises due to the singular nature of log-domain circuits. Damping can be performed by feeding the output of a system back to its input, as is shown in the log-domain signal-flow graph of Figure 2-8.

This results in the circuit shown in Figure 2-8(a) which can be considerably simplified. Because the damped section of the circuit is simply another instance of the logdomain cell, we can replace the appropriate variables in Eq. (2.8) by the appropriate variables from Figure 2-8(a). 


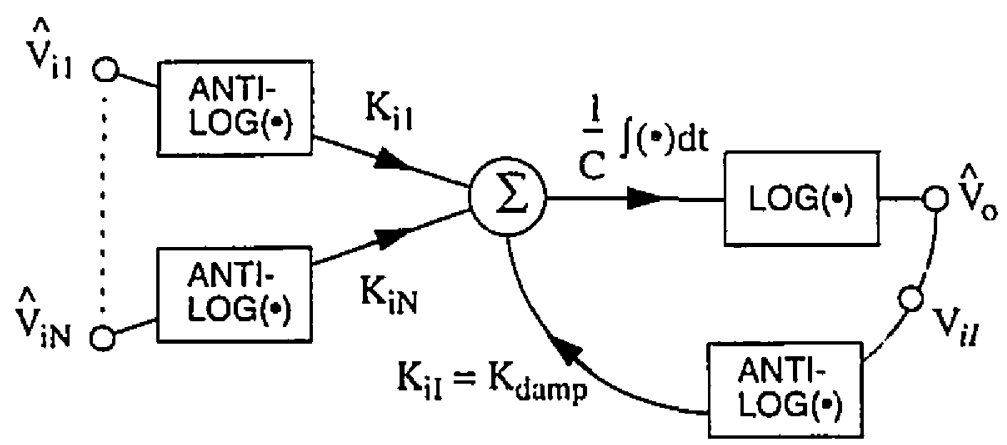

Figure 2-8: SFG of a log-domain integrator with damping

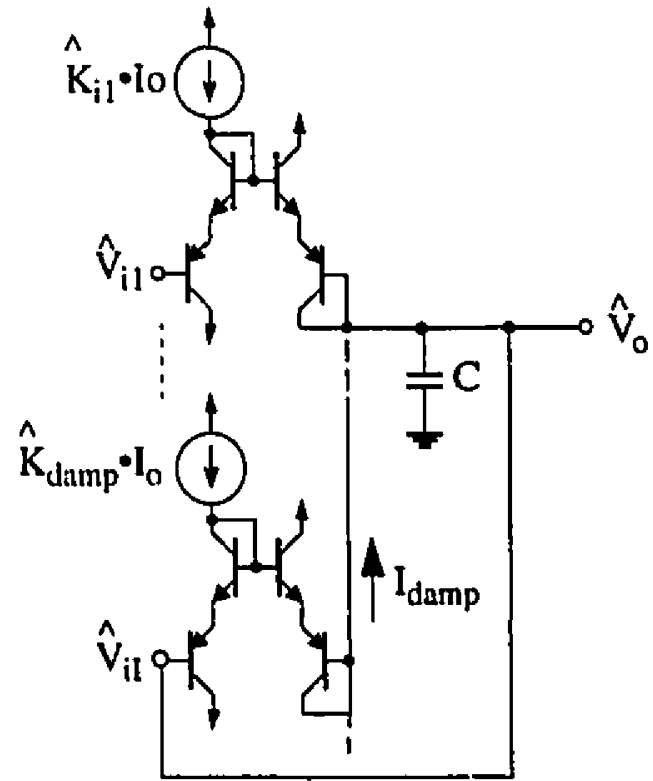

(a)

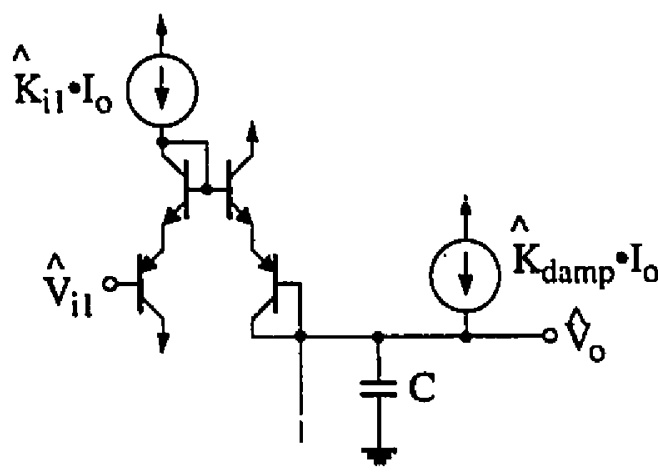

(b)

Figure 2-8: Two equivalent circuits for the damped log-domain integrator 
This gives,

$$
I_{\text {damp }}=\hat{K}_{\text {damp }} \cdot I_{0} \cdot e^{\left(\hat{v}_{n}-\hat{v}_{1}\right) / 2 v_{r}}
$$

which simplifies to,

$$
I_{d a m p}=\hat{K}_{d a m p} \cdot I_{o}
$$

In other words, damping can be represented by a simple current source, as shown in Figure 2-8(b). Note that to change the sign of the damping one simply needs to reverse the current source.

\subsubsection{Input and Output Stages}

We have already seen that in order to maintain the linearity of a log-domain system, a $L O G$ block must be added at the input along with an $A N T I-L O G$ block at the output. These blocks can be implemented using the log-domain cell described in Section 2.1. This procedure will now be demonstrated for the simple log-domain system shown in Figure 2-9. The reader will find that the techniques shown here are applicable to all of the circuits shown in this work.

Replacing the different blocks in Figure 2-9 by their appropriate circuits gives the system shown in Figure 2-10(a). Experienced circuit designers will note that there is at

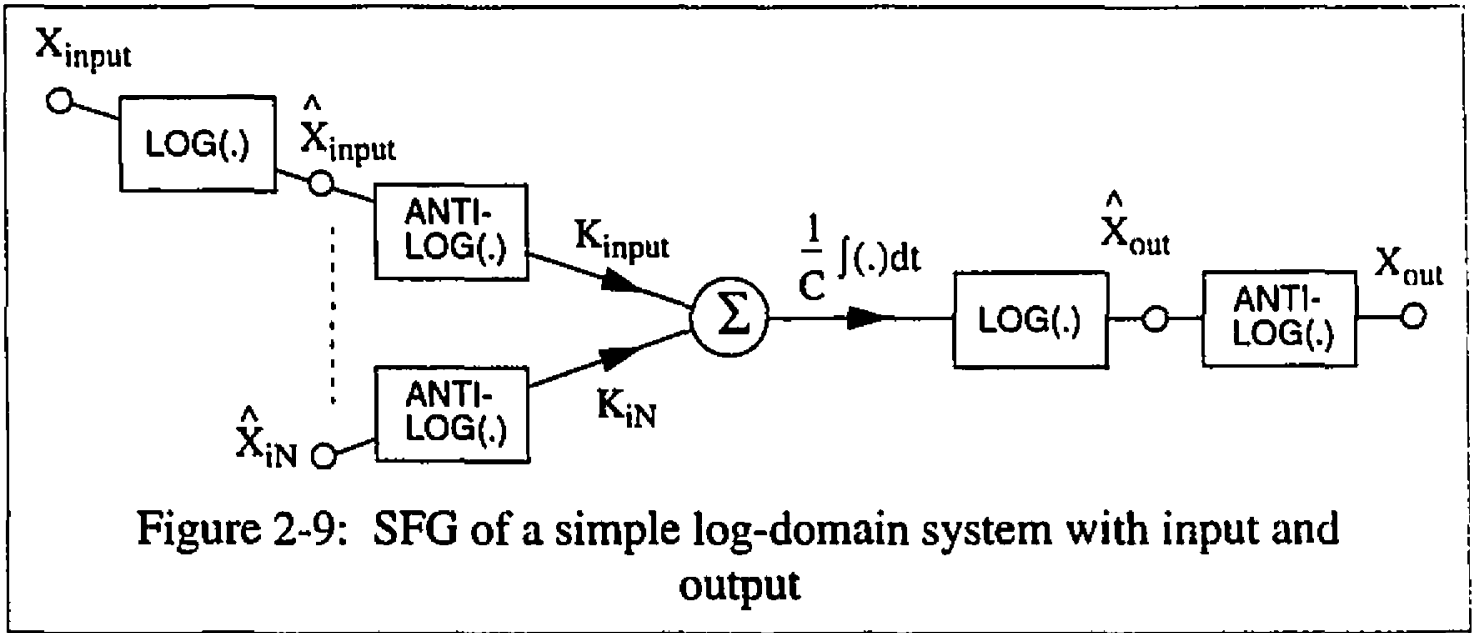


certain amount of redundancy between the $L O G$ section at the input and the inverting integrator. We now perform some algebraic manipulation to implement a more efficient circuit.

From the basic log-domain cell, we can write,

$$
\hat{V}_{\text {input }}=2 V_{T} \cdot \ln \left[\frac{I_{\text {input }}}{I_{o}}\right]
$$

Solving for the portion of the current flowing into the capacitor from the input gives,

$$
I_{x}=\hat{K}_{\text {input }} \cdot I_{0} \cdot e^{\left(\hat{V}_{\text {input }}-\hat{V}_{10}\right) / 2 V_{T}}
$$

Substituting Eq. (2.28) into Eq. (2.29) and simplifying gives:

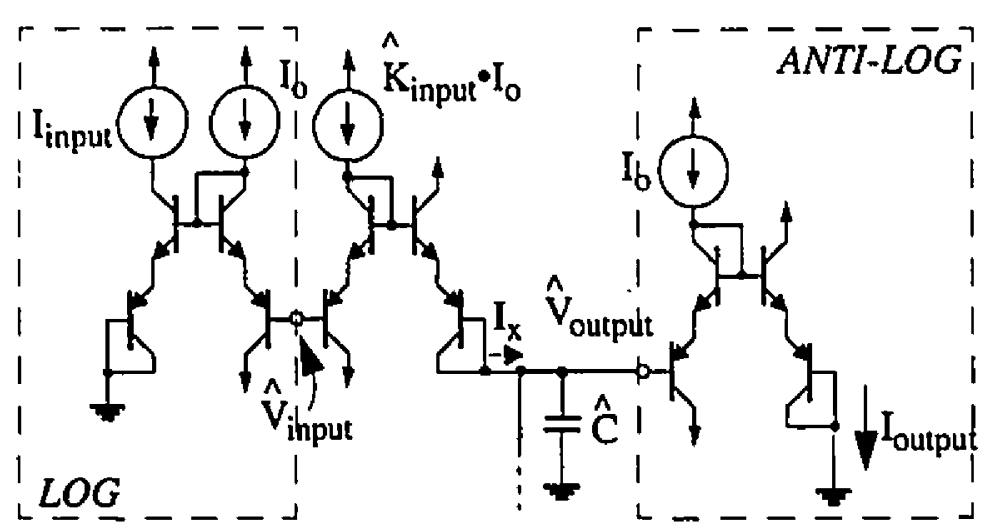

(a)

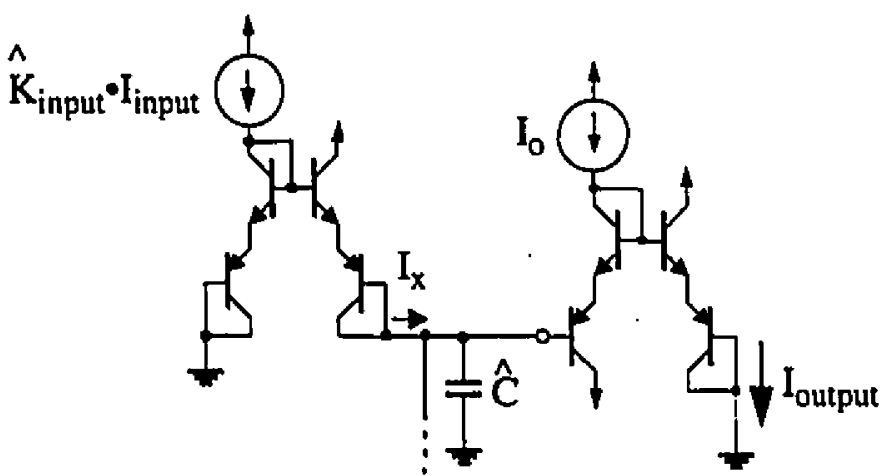

(b)

Figure 2-10: Circuit for the implementation of a simple system with input and output (b) Simplified circuit 


$$
I_{x}=\hat{K}_{\text {input }} I_{\text {input }} \cdot e^{-\dot{V}_{*} / 2 V_{r}}
$$

Again, this is a circuit which can be implemented using the basic cell, ats shown in Figure 2-10(b).

\subsection{Summary}

We have introduced a set of log-domain integrators which can be used for filter design. Because of the non-linear nature of these blocks, a unique linearization procedure was introduced. Chapter 3 will outline a general method for the design of log-domain filters which uses these blocks and which will be used to construct a fifth-order Chebyshev filter. 


\section{$\overline{\text { CHAPTER } 3}$ The Design of All-Pole Lowpass}

\section{Filters}

This chapter will begin with a quick review of the operational simulation of LC ladders. We will show how to transform a traditional linear signal-flow graph into a logdomain signal-flow graph thus putting it in a form which can be implemented using the circuits introduced in the previous chapter. The complete method will be illustrated through the design of a fifth-order Chebyshev filter. The performance of the filter will then be verified using $H S P I C E$ simulation.

\subsection{The Design Method}

The traditional steps for the design of filters based on the operational simulation of LC ladders are as follows:

4. Find an LC ladder which meets the desired filter specifications

5. Draw the SFG which corresponds to the LC ladder

6. Implement the SFG using the desired technology

Examples of technologies which are popular for continuous-time filter design include 
active-RC circuits, $g_{m}-C$ circuits and MOS-C circuits. Log-domain filters will be constructed using the log-domain building blocks described in the previous chapter. Due to the unique nature of these blocks, the signal-flow graph must be modified in order to allow it to be implemented by these non-linear circuits. The steps for the design of a logdomain filter, can be summarized as follows:

1. Find an LC ladder which meets the desired filter specifications

2. Draw the SFG which corresponds to the LC ladder

3. Modify the SFG according to the rules given in Section 3.2

4. Implement the SFG using the log-domain building blocks

\subsection{Modifying the Signal-Flow Graph}

Once an LC ladder has been found which meets the desired filter specifications the designer then draws the signal-flow graph which corresponds to that ladder [26]. In this section, we will show how to modify a traditional signal-flow graph to make it compatible with the log-domain systems described previously. This consists of adding the appropriate $L O G$ and $A N T I-L O G$ blocks while maintaining an overall linear transfer function.

Some simple rules for transforming a linear signal-flow graph into a log-domain signal-flow graph are shown below.

1. Place a $L O G$ block after each integrator.

2. Place an $A N T I-L O G$ block at the input to each summer (before the multiplier)

3. Place an $A N T I-L O G$ block at the output of the system 
4. Place a $L O G$ block at the input to the system

This procedure is illustrated for the signal-flow graph shown in Figure 3-1(a), with the result shown in Figure 3-1(b). By examining the final log-domain signal-fiow graph we can see how the overall transfer function has been maintained. Each $A N T I-L O G$ block is immediately followed by a $L O G$ block, therefore due to the inverse nature of these functions the operation of the original system has been conserved. The highlighted areas represent typical log-domain building blocks with $A N T I-L O G$ blocks at the input and a $L O G$ block at the output. Note also the $L O G$ block at the input to the system and the $A N T I-$ $L O G$ block at the output.

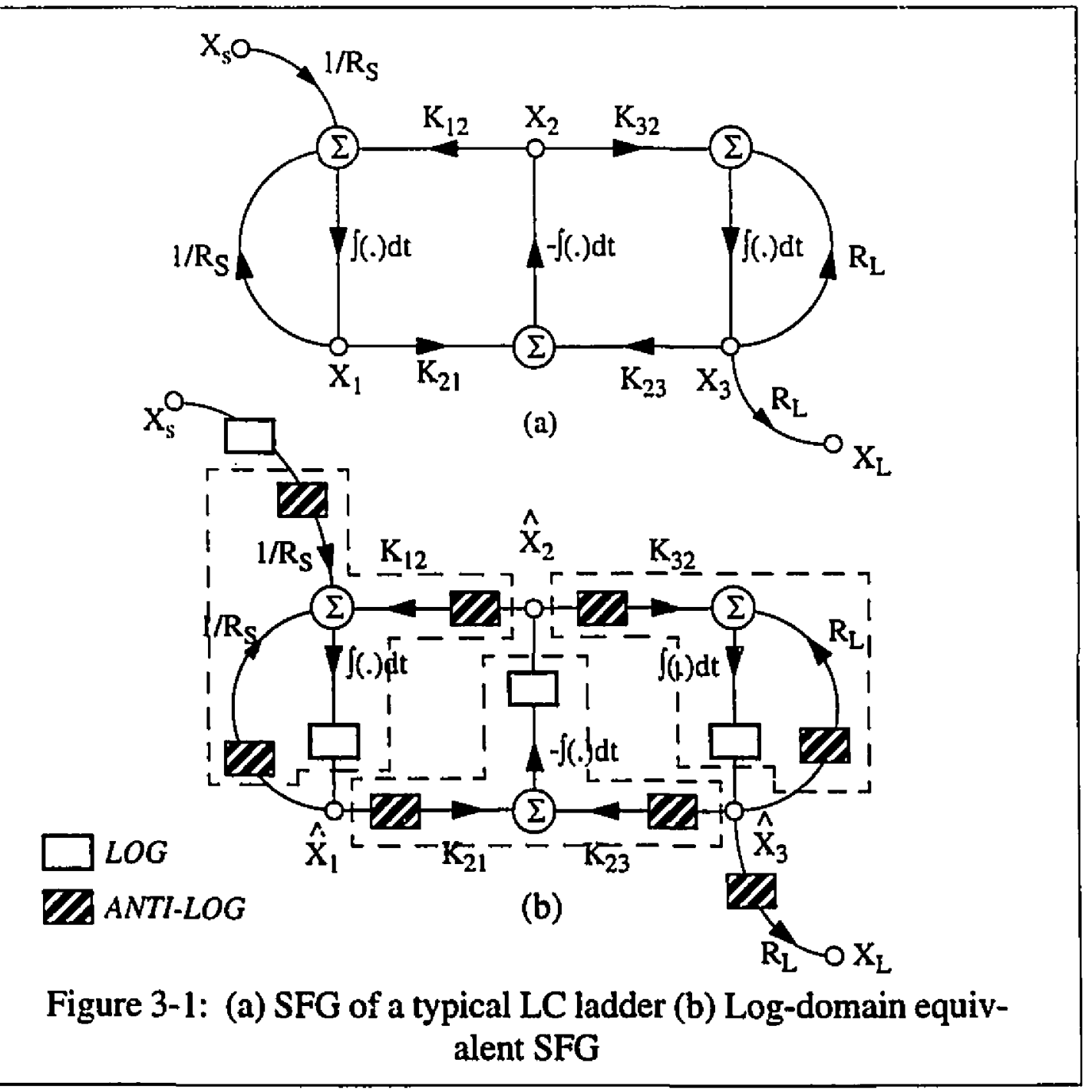




\subsection{A Fifth-Order Chebyshev Filter Design Example}

\subsubsection{The LC Ladder}

The first step in the design of any filter based on the operational simulation of LC ladders is to find the ladder which meets the desired specifications. In this case, we wish to design a fifth-order Chebyshev filter which has:

$$
\begin{gathered}
\text { Cutoff frequency: } f_{c}=100 \mathrm{kHz} \\
\text { Ripple width }=1 \mathrm{~dB}
\end{gathered}
$$

The corresponding LC ladder can be found either through look-up tables $[6,23]$ or through the use of a filter design package. Here the program filtorX [25] was used and resulted in the ladder shown in Figure 3-2. The component values are given in Table 3-1.

\begin{tabular}{|c|c|c|c|}
\hline Name & \multicolumn{1}{|c|}{ Value } & Name & Value \\
\hline \hline $\mathrm{R}_{\mathrm{S}}$ & $1 \Omega$ & $\mathrm{L}_{4}$ & $1.73654 \mu \mathrm{H}$ \\
\hline $\mathrm{C}_{1}$ & $3.39780 \mu \mathrm{F}$ & $\mathrm{C}_{5}$ & $3.39780 \mu \mathrm{F}$ \\
\hline $\mathrm{L}_{2}$ & $1.73654 \mu \mathrm{H}$ & $\mathrm{R}_{\mathrm{L}}$ & $1 \Omega$ \\
\hline $\mathrm{C}_{3}$ & $4.77608 \mu \mathrm{F}$ & & \\
\hline
\end{tabular}

Table 3-1: Component values for the LC ladder

\subsubsection{The Corresponding SFG}

A signal-fiow graph which corresponds to the LC ladder must now be drawn.

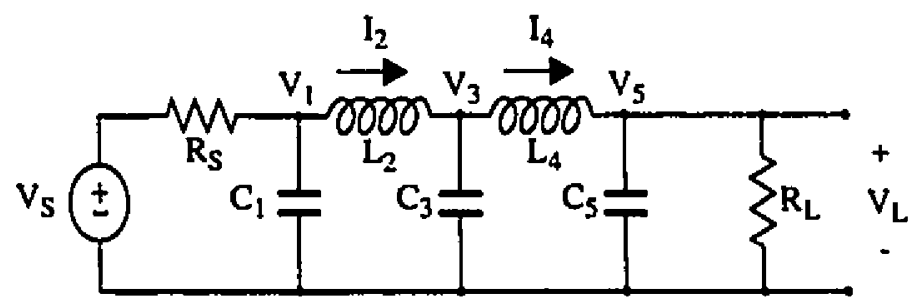

Figure 3-2: LC ladder for a 5th-order Chebyshev filter 
This is most easily accomplished by using modified nodal analysis $[27,28]$. First a current is assigned to each of the inductors in the LC ladder and each node is given a different voltage variable, as shown in Figure 3-2. We then write impedance descriptions for each inductor in terms of its node voltages and the inductor current. This results in the following two equations:

$$
\begin{aligned}
& L_{2} \frac{d}{d t} I_{2}=V_{1}-V_{3} \\
& L_{4} \frac{d}{d t} I_{4}=V_{3}-V_{5}
\end{aligned}
$$

Nexl, we apply KCL to the previously labelled voltage nodes and derive a set of equations relating the node voltages, the inductor currents and the input. For the LC ladder of Figure 3-2 this results in the equations:

$$
\begin{gathered}
C_{1} \frac{d}{d t} V_{1}=\frac{V_{S}}{R_{S}}-\frac{V_{1}}{R_{S}}-I_{2} \\
C_{3} \frac{d}{d t} V_{3}=I_{2}-I_{4} \\
C_{5} \frac{d}{d t} V_{5}=I_{4}-\frac{V_{5}}{R_{L}}
\end{gathered}
$$

We also note that,

$$
V_{L}=V_{5}
$$

The set of equations (3.1) to (3.6) completely specifies the LC ladder. In order to remain consistent with signal-flow graph convention let us define a set of general variables which will correspond to the voltages and currents denoted previously. In other words, we define,

$$
X_{s} \Leftrightarrow V_{s}, X_{1} \Leftrightarrow V_{1}, X_{2} \Leftrightarrow I_{2}, X_{3} \Leftrightarrow V_{3}
$$




$$
X_{4} \Leftrightarrow I_{4}, X_{5} \Leftrightarrow V_{5}, X_{L} \Leftrightarrow V_{L}
$$

We may now draw the signal-flow graph which corresponds to the LC ladder using fle six equations defined previously and the variable correspondences given above. This is shown in Figure 3-3(a).

The signal-flow graph is then modified according to the rules given in Section 2.4. The log-domain signal-flow graph is shown in Figure 3-3(b). Note how the operation of the original signal-flow graph has been maintained due to the inverse nature of the $L O G$ and $A N T I-L O G$ functions.

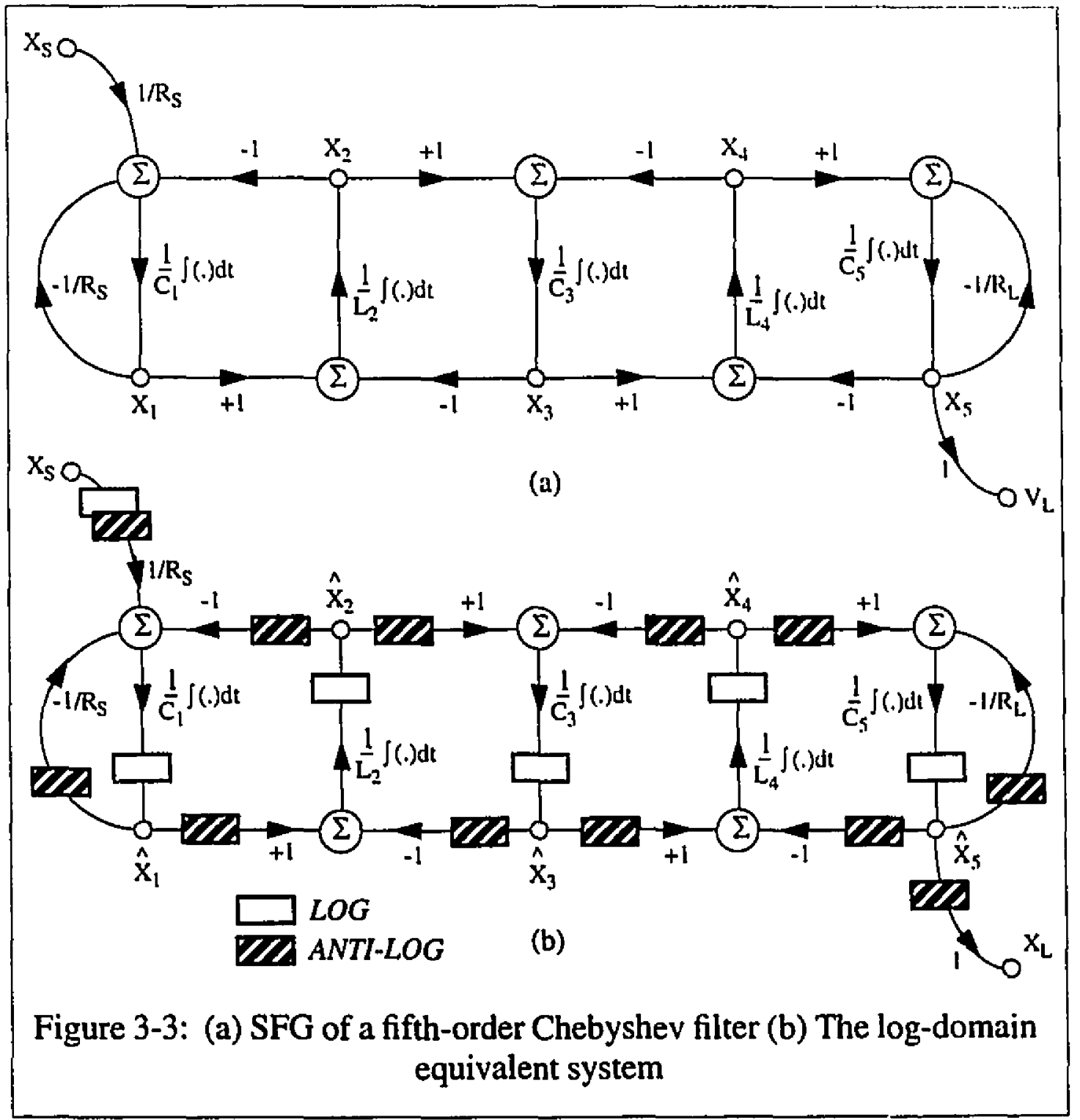




\subsubsection{The Complete Chebyshev Log-Domain Filter}

The final step in the design of the lug filter involves replacing the different components of the signal-flow graph shown in Figure 3-3(b) with the circuits introduced in Section 2.4. This results in the complete log-domain circuit shown in Figure 3-4 on the following page. The scaling factor $I_{o} / 2 V_{T}$ which is necessary to maintain the equivalence between the log-domain integrator and the corresponding linear integrator was accounted for by scaling the original LC ladder component values by $I_{o} / 2 V_{T}$. The new component values are given in Table 3-2. Note that the filter could also have been implemented by keeping the same component values as the LC ladder and scaling the bias currents by the factor $l_{o} / 2 V_{T}$. This will be confirmed when we examine the tunability of this filter later in the chapter.

\begin{tabular}{|c|l|c|l|}
\hline Name & \multicolumn{1}{|c|}{ Value } & Name & \multicolumn{1}{|c|}{ Value } \\
\hline \hline $\mathrm{R}_{\mathrm{S}}$ & $1 \Omega$ & $C_{L_{4}}$ & $3.47308 \mathrm{nF}$ \\
\hline$C_{C_{1}}$ & $0.79560 \mathrm{nF}$ & $C_{C_{S}}$ & $6.79560 \mathrm{nF}$ \\
\hline$C_{L_{2}}$ & $3.47308 \mathrm{nF}$ & $\mathrm{R}_{\mathrm{L}}$ & $1 \Omega$ \\
\hline$C_{C_{3}}$ & $9.55216 \mathrm{nF}$ & & \\
\hline
\end{tabular}

Table 3-2: Component values for the 5th-order Chebyshev logdomain filter circuit 
Chapter 3: The Design of All-Pole Lowpass Fillers

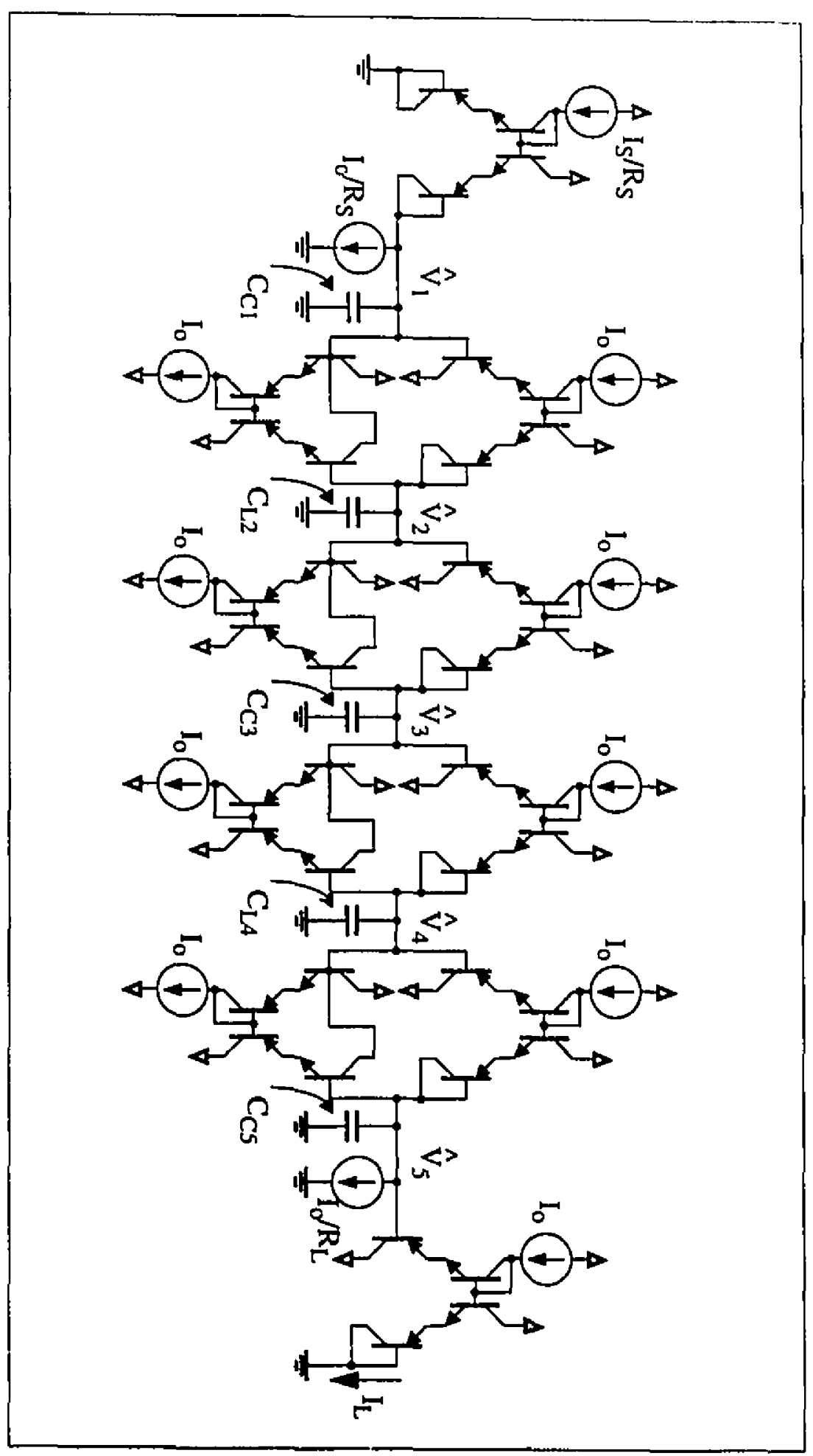

Figure 3-4: Circuit diagram for the fifth-order Chebyshev log filter 
Now that a complete log-domain filter has been designed we take a minute to examine the physical nature of this circuit. Like in the LC ladder, there exists a correspondence between the variables in the log-domain signal-flow graph and the physical voltages and currents in the circuit of Figure 3-4. These are as follows:

$$
\begin{gathered}
X_{S} \Leftrightarrow I_{S}, \hat{X}_{1} \Leftrightarrow \hat{v}_{1}, \hat{X}_{2} \Leftrightarrow \hat{V}_{2}, \hat{X}_{3} \Leftrightarrow \hat{V}_{3} \\
\hat{X}_{4} \Leftrightarrow \hat{V}_{4}, \hat{X}_{5} \Leftrightarrow \hat{v}_{5}, X_{L} \Leftrightarrow I_{L}
\end{gathered}
$$

It has already been shown that the original signal-flow graph representation of the LC ladder and the log-domain signal-flow graph are equivalent. It is interesting to note that while the input-output signals in the LC ladder are represented by voltages, the input and output of the log-domain filter are both currents. This has led some to characterize the log-domain filter as a current-mode circuit.

What the method of design proposed in this thesis has shown us is that the key nodes in the log-domain signal-flow graph $\left(\hat{X}_{1}, \hat{X}_{2}, \ldots\right)$ are physically represented by voltages. In fact, the input and output are only currents due to the LOG and ANTI-LOG functions which have been added to preserve the linearity of the system. This would indicate to some that this is a voltage-mode circuit. Suffice to say that it is always difficult to classify circuits in this manner due to the fundamental relationship between voltage and

current (e.g.. Ohm's law). The important thing is to realize that this circuit possesses many of the advantages which are often associated with current-mode circuits, namely the fact that impedances along the signal path are low and that there all small voltage swings at the key nodes.

\subsection{Simulation Results}

The performance of the fifth-order Chebyshev filter will now be measured through HSPICE simulations $[29,30]$. The tests that will be performed fall under two main 
categories: frequency behavior and linearity. First, the overail frequency performance of the filter will be measured by comparing the frequency response of the log-domain circuit to the LC ladder that it was designed to implement. The tunability and high-frequency performance of the filter will also be verified since these are two of the most important properties of these circuits.

The second big area of interest is the linearity of these lilters. Although lincirity is always an important property of electronic circuits, it is of particular importance here due to the unique linearization procedures used in these circuits. In order to verify this area of the filter's performance several different distortion tests will be performed.

\subsubsection{Frequency Response}

The traditional method for simulating the frequency response of a circuil using HSPICE is to perform AC analysis. HSPICE does this type of analysis by tirst solving for the DC operating point of the circuit, then it determines linearized small-signal models for the different components in the circuit. Because this approach negates the essential translinear nature of the bipolar transistor, it is not particularly suited to testing the logdomain technique. A better approach is to perform multitone analysis.

\subsubsection{Multitone Analysis}

Multitone analysis is popular in the testing community and involves finding the spectral response of a circuit stimulated by an input consisting of many sinusoidal tones (a multitone input). Appendix A describes the multitone simulation method used in this thesis. This novel approach combines multitone testing with HSPICE transient analysis. Because of the large-signal nature of HSPICE transient analysis, the circuit is simulated without small-signal approximation. In addition to allowing the measurement of frequency response, multitone analysis can also be used for distortion measurements.

The fifth-order Chebyshev filter was simulated using a 16 tone multitone input, 
where cach tone had an amplitude of $40 \mu \mathrm{A}$. The sampling frequency was chosen to be $750 \mathrm{kHz}$ and the frequencies of the input tones were chosen according to the method described in Appendix A. The results are shown in Figure 3-5. The simulations were performed using both ideal transistors and transistor models for a bipolar analog array provided by the Gennum Corporation [31]. The complete transistor models can be found in Appendix C. The circuit tested using ideal transistors had a cutoff frequency and a passbund ripple very close to that of the $L C$ ladder and met the desired specifications. This confirms that the theory developed previously is accurate. If we have a device which can provide a perfect exponential function, like an ideal bipolar transistor, then we will get the desired frequency response.

The performance of the circuit modelled with Gennum transistors shows a slight change in the passband ripple and a shift of the cutoff frequency by about $20 \mathrm{kHz}$. This is primarily due to the finite $\beta$ which affects the bias current of the transistors, and in turn influences the cutoff frequency of the filter. This characteristic of log-domain filters is what makes these circuits tunable and should allow us to establish a tuning circuit which wil! account for this shift in frequency. Tunability will be discussed in some depth later in this section.

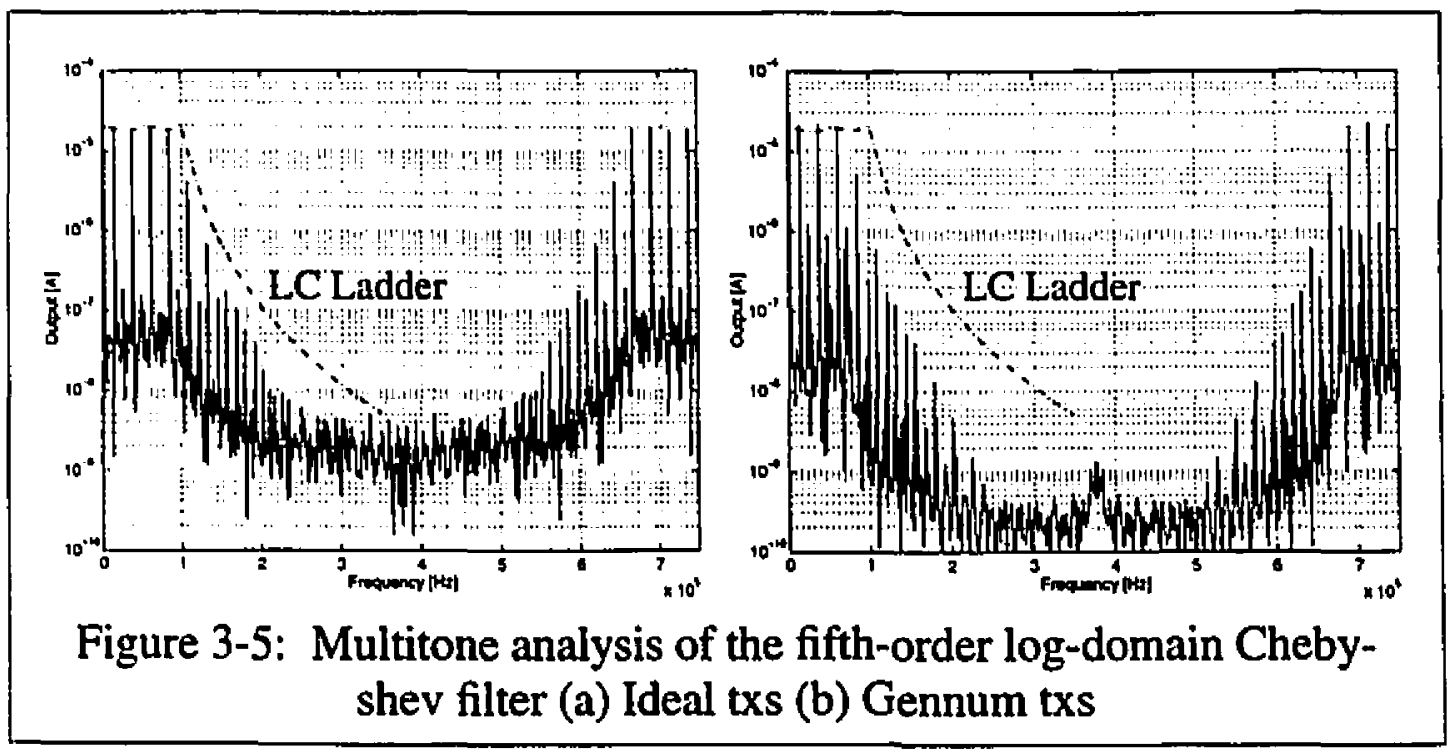




\subsubsection{AC Analysis}

Multitone analysis is the best method for testing log-domain filters since AC analysis does not account for the transinear nature of these circuits. However, once the operation of the circuit has been confirmed through multitone analysis, AC analysis can then be used as a quicker way to get the frequency response. This is because the $\mathrm{AC}$. analysis results are not wrong, it is just that they are only limited to small signal inputs.

In order to confirm that the AC results are the same as those obtained from multitone analysis, we perform $\mathrm{AC}$ analysis on the fifth-order Chebyshev filter derived previously. The magnitude response of the filter is shown in Figure 3-6. Like in the multitone case, we compare the frequency response of the LC ladder to that of the logdomain filter simulated using both ideal transistors and Gennum transistors. The results are indeed the same as the ones given by multitone analysis. This is not immediately obvious because the frequency axis is logarithmic in this case. However, if we examine the results for the circuit modelled using Gennum transistors we can see the same loss of symmetry in the passband and the $20 \mathrm{kHz}$ shift in the cutoff frequency. Again the circuit modelled using ideal transistors gives an almost identical response to that of the LC
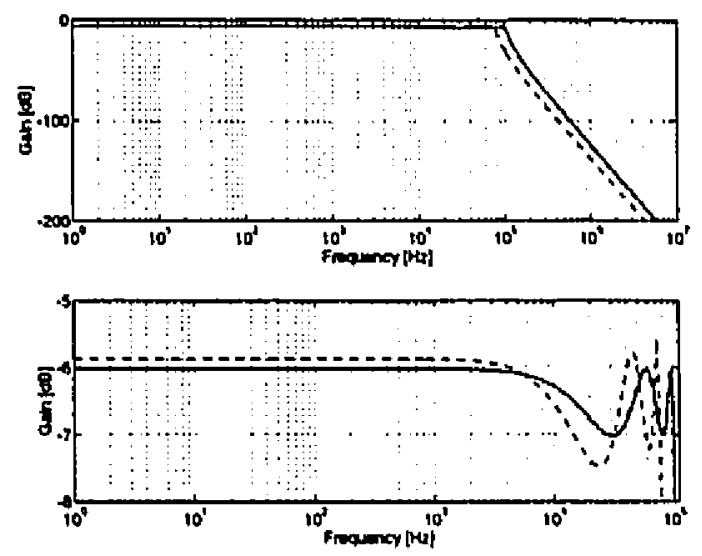

Figure 3-6: AC analysis of the 5th-order Chebyshev log-domain filter (a) Full frequency (b) Passband 
ladder.

\subsubsection{Tunability}

We have already shown that in order to maintain the equivalence between a logdomain system and its corresponding linear system ( $\mathrm{LC}$ ladder), then either the capacitance values or the bias currents must be scaled by the factor $I_{o} / 2 V_{T}$. To fail to do so will have an effect on the frequency behavior of the log-domain filter. While this adds an extra element of complexity to the design of log filters, it is also what makes these circuits easily turable.

Let us examine the frequency response of the Chebyshev filter if we keep the Capacitances in the circuit constant and vary all of the bias currents simultaneously. Figure 3-7 shows $\mathrm{AC}$ analysis results for the circuit simulated with bias levels of $0.1 \mu \mathrm{A}, 1 \mu \mathrm{A}$, $10 \mu \mathrm{A}$ and $100 \mu \mathrm{A}$. The transistors were modelled with Gennum models. The input signal level was chosen such that it was of the same order as the bias current and only the gain was plotted. Figure 3-7 confirms that the cutoff frequency is directly proportional to the bias current.

The disadvantage of this aspect of the log filter's performance is that any change in the bias currents will lead to a change in the cutoff frequency. We have already seen

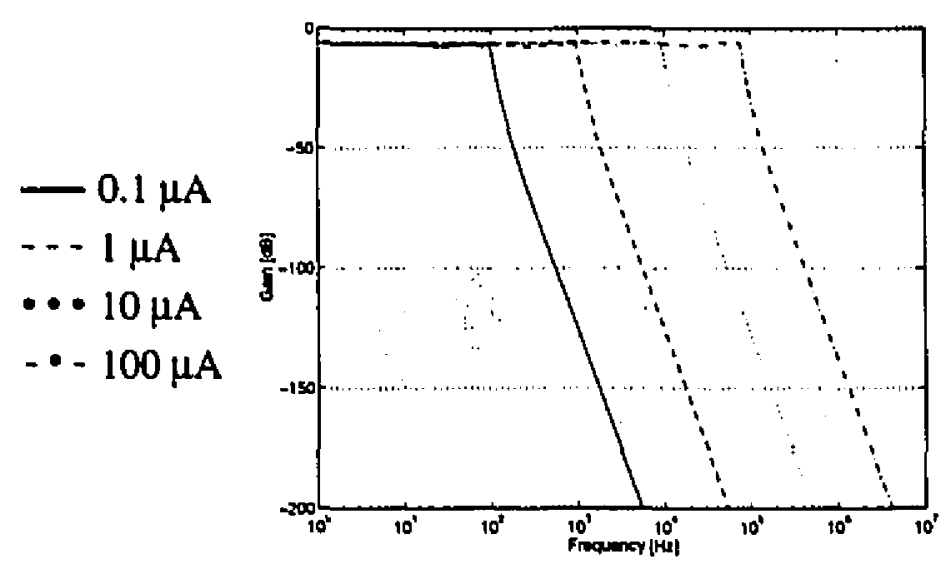

Figure 3-7: Tunability of the log-domain filter 
how the finite $\beta$ associated with real transistors leads to base current loss and hence 10 changes in the cutotf frequency. The solution is to use a tuning scheme which will allow the designer to precisely set the cutoff frequency of the filter to its desired value by tine tuning the bias currents.

A second feature of these circuits which arises due to the scaling factor $l_{1} / 2 V_{r}$ is that the cutoff frequency is dependent on the thermal voltage, $V_{T}$. Because of the strong relationship between the thermal voltage and temperature, this makes the frequency behavior of the $\log$ filter temperature dependent. However, since we know of the linear behavior of the filter with temperature, this problem can be easily remedied. One solution would be to make the current sources inversely proportional to temperature using a scheme similar to the one used in band-gap references [12]

\subsection{J.4 High-Frequency Performance}

Another feature of the log-domain filter which will be explored is to measure the maximum cutoff frequency which can be achieved before the frequency behavior begins to break down. The frequency behavior of any transistor circuit will eventually be limited by the $f_{t}$ of the slowest transistor in the circuit. In order to test the high-speed performance of this technique independent of the transistors which are used, we will measure how close to the $f_{t}$ of the slowest transistor the circuit can be run.

The reason that the log-domain filter shows promise in the area of high-speed filter design is that all of the nodes along the signal path are low impedance. As a result, the poles which occur due the parasitic capacitances associated with the transistors will be placed at higher frequencies than if they were in a circuit with high impedance nodes along the signal path. Traditional filter designs tend to be made from voltage-mode circuits which have numerous high impedance nodes.

In order to measure the high-speed performance of the log-domain filter, the cutoff frequency will be varied by decreasing the capacitor values in the circuit. As in the 


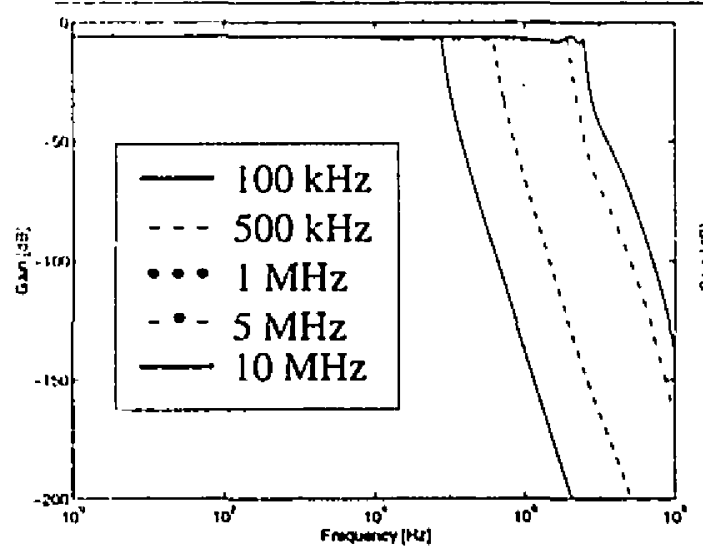

(a)

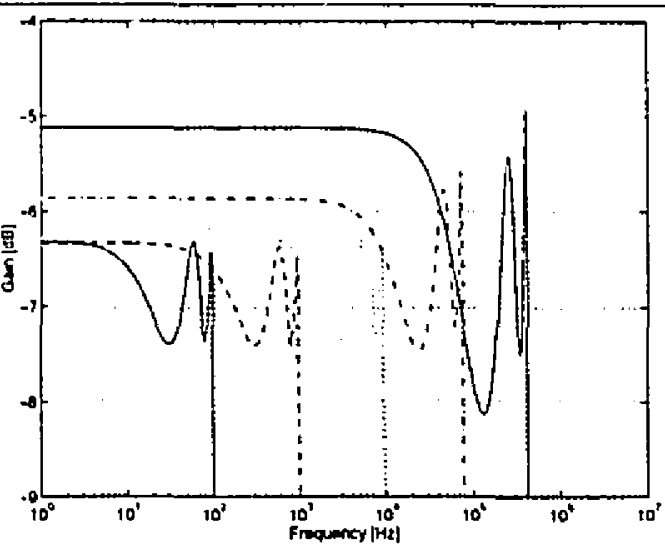

(b)

Figure 3-8: High-frequency performance of the log filter (a) Full frequency range (b) Passband

LC ladder, the cutoff frequency of the filter will increase accordingly. The cutoff frequency will be increased until the frequency behavior of the filter begins to lose its shape in the passband. This will give us the maximum operating frequency of the circuit. The maximum bandwidth of the circuit will then be reported as a percentage of the $f_{t}$ of the slowest transistor in the circuit.

Figure 3-8 shows the frequency behavior of the log-domain filter designed for five different cutoff frequencies. The different cutoff frequencies are specified to the left of the graph. The circuit was again modelled using Gennum transistor models. The Gennum transistors have $f_{i}$ 's of $2.5 \mathrm{GHz}$ for the NPN transistors and $10 \mathrm{MHz}$ for the PNP transistors. The results show that the log filters maintain their frequency behavior up to approximately $1 \mathrm{MHz}$, or $1 / 10$ th of the $f_{\mathrm{t}}$ of the slowest transistor. This confirms that logdomain filtering is indeed a relatively fast filtering scheme.

\subsubsection{Distortion Measurements}

The log-domain filter, like other transistor circuits, is susceptible to distortion due to the non-linear nature of the bipolar transistor. Distortion measurement is of particular importance in this type of filter because of the unique approach used for linearizing the 
circuit. Simulated distortion measurements will be performed using the multitone technique described in Appendix A. The input will be composed of either one or two tones depending on the distortion test. A single tone allows us to measure total harmonic distortion (THD) while two tones are used for intermodulation distortion measurement (IMD).

\subsubsection{The Intercept Concept}

When measuring the distortion level associated with a circuit it is important to specify the input level at which it was measured. For example, one circuit may specify a THD level of $-60 \mathrm{~dB}$ while another might specify a distortion level of $-40 \mathrm{~dB}$ but unless the same input level was used, no meaningful comparison can be made. One way to ensure that a fair comparison is made between different circuits is to measure the distortion and compare it to circuits' intercept points [32]. An intercept point is a relative measure which is defined as the level where the output power of the fundamental is equal to the output power of a given harmonic. There will be an intercept point for each harmonic but it is customary to only give intercept plots for the first two harmonics. Figure 3-9 shows the intercept plots for the fifth-order Chebyshev filter simulated using both ideal and Gennum transistors. The input was a single $1 \mathrm{kHz}$ sinusoidal tone such that it was well within the

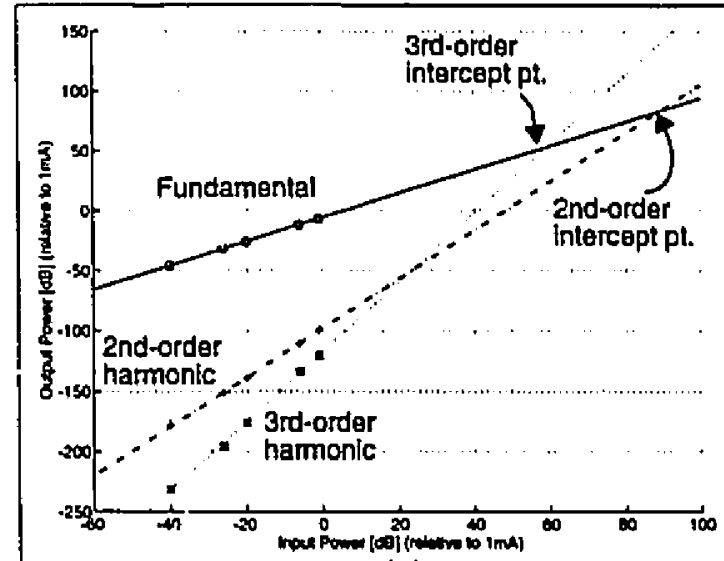

(a)

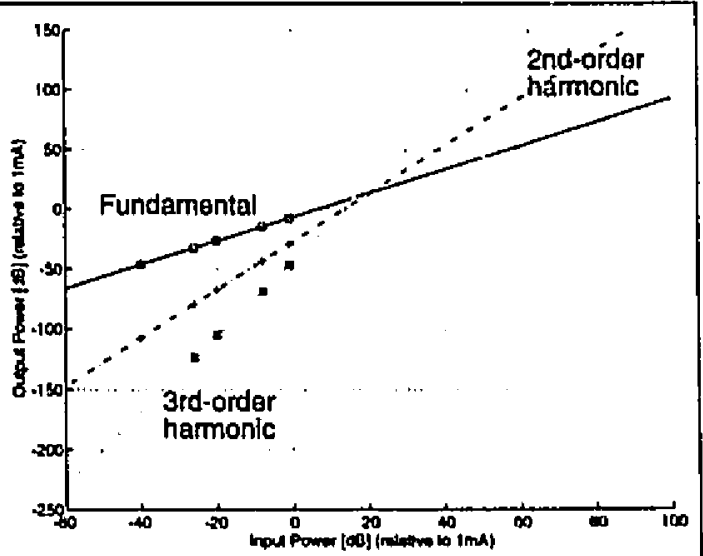

(b)

Figure 3-9: intercept plots for the log-domain filter (a) Ideal txs (b) Gennum txs 
passband of the circuit. The relative power measurements are given with respect to $1 \mathrm{~mA}$, as this was the maximum current used in the experiment.

$$
\text { Power }=20 \cdot \log 10\left[\frac{I_{m e a s}}{1 m A}\right]
$$

Table 3-3 gives numerical values for the intercept points. The intercept points are much lower for the Gennum transistors than for ideal transistors which indicates that the distortion is greater when real transistors are present. This is to be expected since the linearity of iog-domain filters is related to the transistors ability to perform the inverse functions, $L O G$ and $A N T I-L O G$. The more ideal the transistors, the better matched the functions will be.

\begin{tabular}{|c|l|l|l|}
\hline Transistors & Intercept Point & Input Power & Output Power \\
\hline \hline \multirow{2}{*}{ Ideal } & 2nd Order & $91.4 \mathrm{~dB}$ & $85.5 \mathrm{~dB}$ \\
\cline { 2 - 4 } & 3rd Order & $61.6 \mathrm{~dB}$ & $55.7 \mathrm{~dB}$ \\
\hline \hline Gennum & 2nd Order & $20.2 \mathrm{~dB}$ & $13.0 \mathrm{~dB}$ \\
\cline { 2 - 4 } & 3rd Order & $17.8 \mathrm{~dB}$ & $10.5 \mathrm{~dB}$ \\
\hline
\end{tabular}

Table 3-3: Intercept points of the log-domain filter

\subsubsection{Total Harmonic Distortion}

Here we present the most traditional measure of distortion in electronic circuits, namely total harmonic distortion or THD. This is a measure of the power of the harmonics to the power of the fundamental and is calculated using the following formula:

$$
T H D=\frac{\sqrt{I_{2}^{2}+I_{3}^{2}+I_{4}^{2}+\ldots}}{I_{1}}
$$

$I_{l}$ is the current magnitude of the fundamental and $I_{2}, I_{3}, \ldots$ are the current magnitudes of the harmonics (with the subscript denoting the corresponding harmonic term). The filter 
was tested using a sinusoidal input signal at a frequency of much less than the cutoff frequency of the filter. This ensures that only very high harmonics will be affected by the filter's frequency response. The input sinusoid had an amplitude of $10 \mu \mathrm{A}$ and a frequency of $1 \mathrm{kHz}$. The spectral response of the filter stimulated by this tone and is shown in Figure 3-10. The frequency response is given for the filter tested using both ideal and Gonnum transistor models. The total harmonic distortion was then measured with the results given in Table $3-4$.

Using ideal transistors we get very little distortion which proves that, despite the non-linearity of the individual integrators in the log-domain filter, we still have a very linear system overall. When real transistors are used the distortion is considerably greater due to the fact that the transistors are no longer acting as perfect exponential functions. Using better transistors than the ones provided by the relatively low-cost Gennum process, one would expect to get distortion levels somewhere between the ideal transistors and the Gennum transistors.

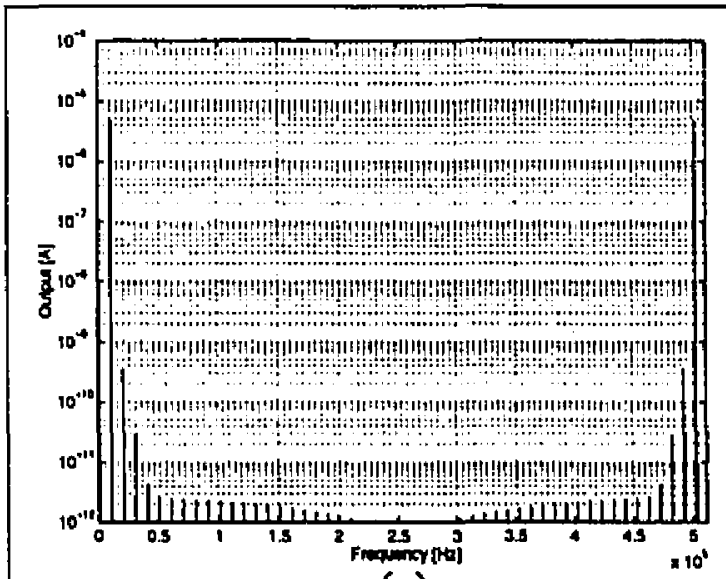

(a)

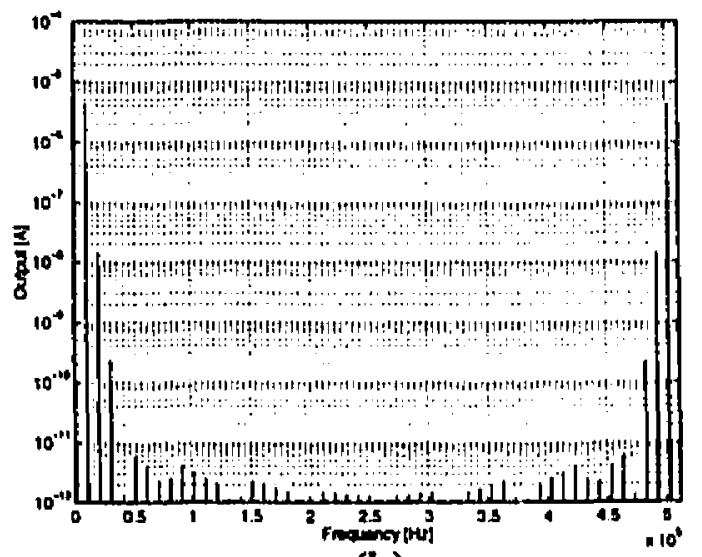

(b)

Figure 3-10: Frequency plots for calculating the THD of the log-domain filter (a) Ideal txs (b) Gennum txs 


\begin{tabular}{|c|c|c|c|}
\hline Transistors & $\mathrm{I}_{\text {in }}$ & THD [\%] & THD [dB] \\
\hline \hline Ideal & $10 \mu \mathrm{A}$ & $0.00739 \%$ & $-82.6 \mathrm{~dB}$ \\
\hline Gennum & $10 \mu \mathrm{A}$ & $0.329 \%$ & $-49.7 \mathrm{~dB}$ \\
\hline
\end{tabular}

Table 3-4: Total harmonic distortion in the log-domain filter

\subsubsection{Intermodulation Distortion}

The final form of distortion measurement which will be made is that of intermodulation distortion (IMD). This involves stimulating a circuit with two tones, usually close in frequency and near the passband edge of the filter. In addition to the usual harmonics present at the output, we expect to see harmonics which satisfy the following criteria:

$$
f_{n m}=\left|n f_{1}+m f_{2}\right|
$$

where $n$ and $m$ are positive integers such that $n+m \leq 3$. Figure 3-11 shows the spectral response of the log filter stimulated by two tones, one at $75 \mathrm{kHz}$ and another at $80 \mathrm{kHz}$. The reader can clearly see the different harmonics created by the mixing of the two different frequencies. A quantitative measure of the intermodulation distortion in the log filter will be given in the experimentad results of Chapter 5 .

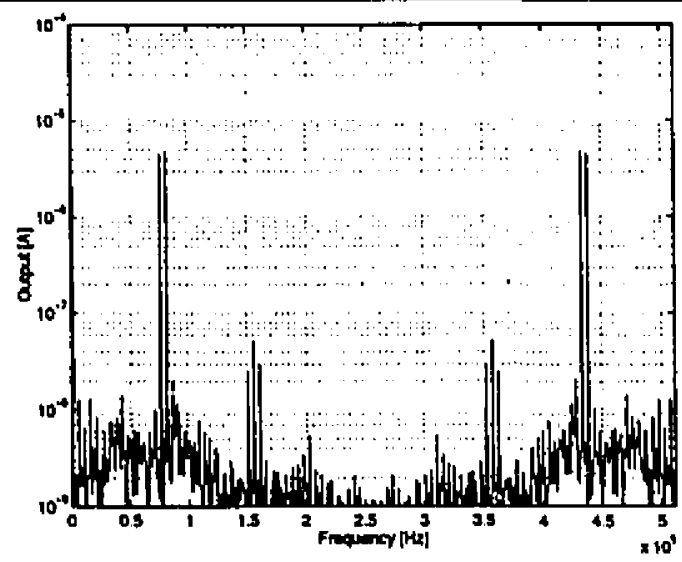

Figure 3-11: Intermodulation distortion in the log-domain filter 


\subsection{Summary}

The frequency response of the filter was identical to that of its LC ladder when ideal transistors were used and only slightly off with real transistor models. The filter showed tunability and high-frequency operation up to $1 / 10$ th of the $f$ of the slowest transistor in the circuit. The simulated distortion levels were comparable to distortion measurements quoted for other filtering techniques [1].

The next chapter will examine the performance of filters with finite transmission zeros, specifically elliptic and bandpass filters. 


\section{CHAPTER 4 Elliptic and Bandpass Filters}

This chapter will introduce two new types of log-domain filters which contain finite transmission zeros; namely elliptic and bandpass filters. Both filters will be derived using the basic techniques described in Chapters 2 and 3. The derivation of the elliptic filter will require an approximation in order to compensate for the fact that no log-domain differentiator has been introduced. As before, HSPICE simulations will be used to confirm that these filters have the proper frequency behavior.

\subsection{Elliptic Filters}

\subsubsection{Derivation of a Third-Order Elliptic Filter}

In order to limit the complexity of the derivation of this filter and the size of the ensuing circuit, the design will be limited to that of a third-order elliptic filter. The filter characteristics are chosen as:

Cutoff frequency: $f_{c}=10 \mathrm{kHz}$

Stopband Region: $\Omega_{\mathrm{s}}=20 \mathrm{kHz}$

Stopband Attenuation $=30 \mathrm{~dB}$

Passband Ripple $=0.5 d B$ 


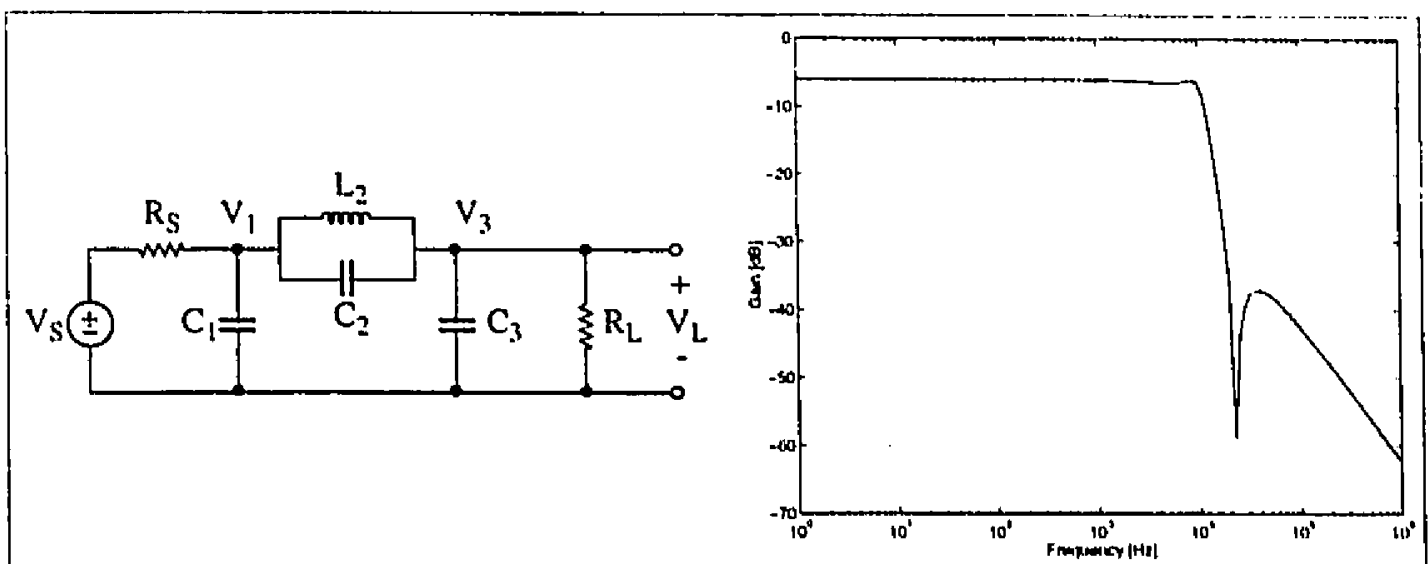

Figure 4-1: (a) LC ladder for a 3rd-order elliptic filter and its associated frequency response

The LC ladder which meets these specifications is shown in Figure 4-1 along with its associated frequency response. As in Chapter 3, the signal-flow graph can be drawn using modified nodal analysis which results in the signal-flow graph of Figure 4-2(a).

As should be expected from the similarity of their LC ladders, the signal-flow graph of the elliptic filter is very much like the one for the Chebyshev filter. The only difference lies in the differentiator which is caused by the series capacitor between nodes $V_{1}$ and $V_{3}$. As will be shown shortly, a single capacitor can be used to perform the differentiator operation in the log-domain filter as long as a simple approximation is made.

The next step in the design of the filter is to redraw the signal-flow graph according to the rules of Chapter 3 (Figure 4-2(b)). The differentiator sections can be left as is without changing the overall linear input-output relationship of the filter. Unfortunately, it is not possible to implement this circuit based solely on the building blocks of Chapter 2 since we have not introduced a log-domain differentiator. However we know that in other filter technologies (active-RC for example), a simple capacitor is used to implement the differentiator. Let us, for the sake of argument, assume that a capacitor placed between nodes $\hat{X}_{1}$ and $\hat{X}_{3}$ of the Chebyshev filter will give the desired 


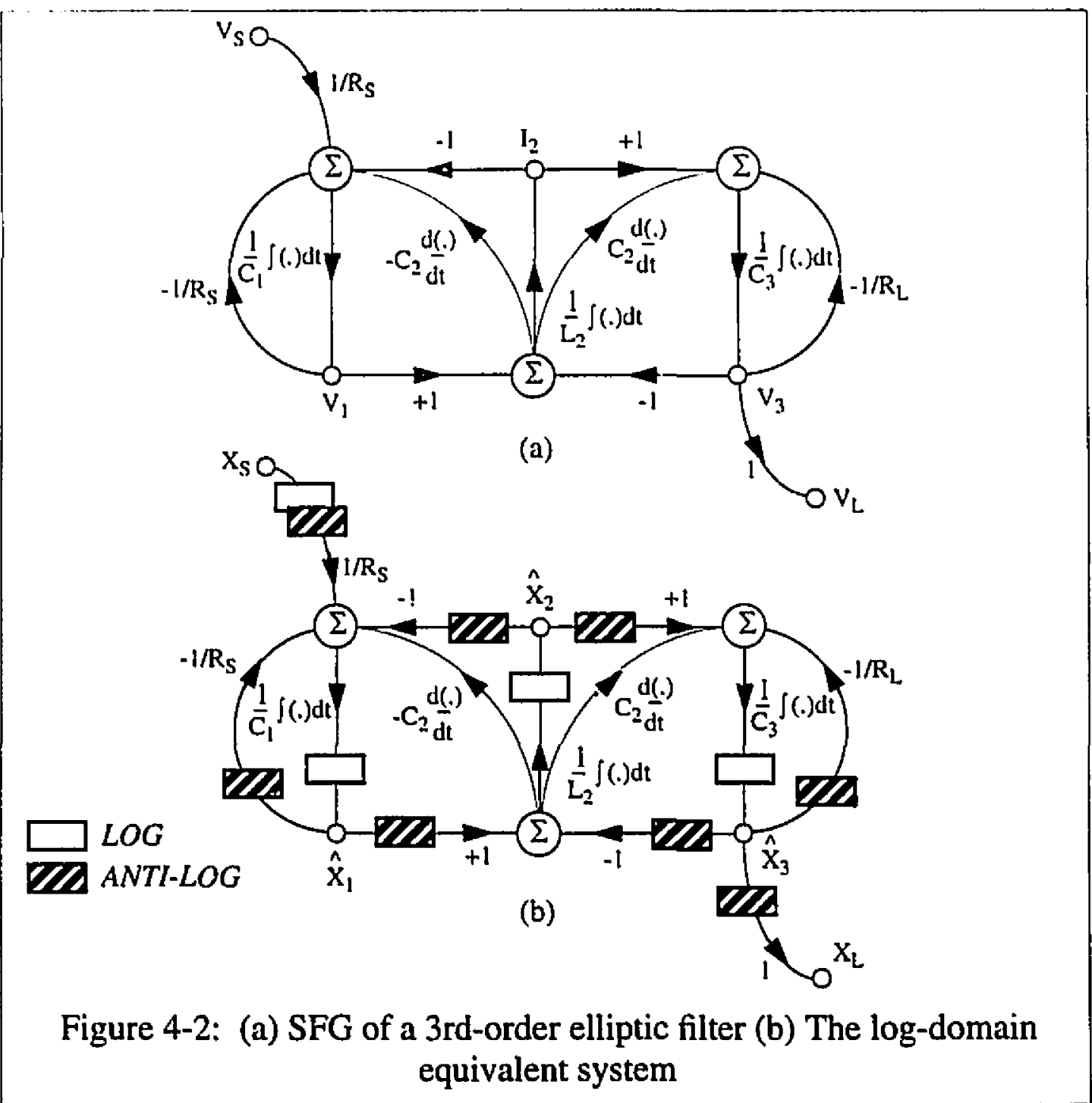

elliptic transfer function. This would result in the circuit shown in Figure 4-3.

In order to verify that the circuit of Figure 4-3 corresponds to the signal-flow graph of Figure 4-2(b) we begin by writing an expression for the current flowing into the capucitor $C_{C 3}$ due to the non-inverting integrator and the capacitor $C_{C 2}$. Note that for the sake of simplicity the current drawn from the current source and from the output section have been omitted. The current through $C_{C 3}$ is thus given by:

$$
C_{C 3} \cdot \frac{d}{d t} \hat{V}_{3}=\hat{K_{23}} \cdot I_{o} e^{\left(\hat{V}_{2}-\hat{v}_{3}\right) / 2 V_{T}}+\ldots+C_{C 2} \cdot \frac{1}{d t}\left(\hat{V}_{1}-\hat{V}_{3}\right)
$$




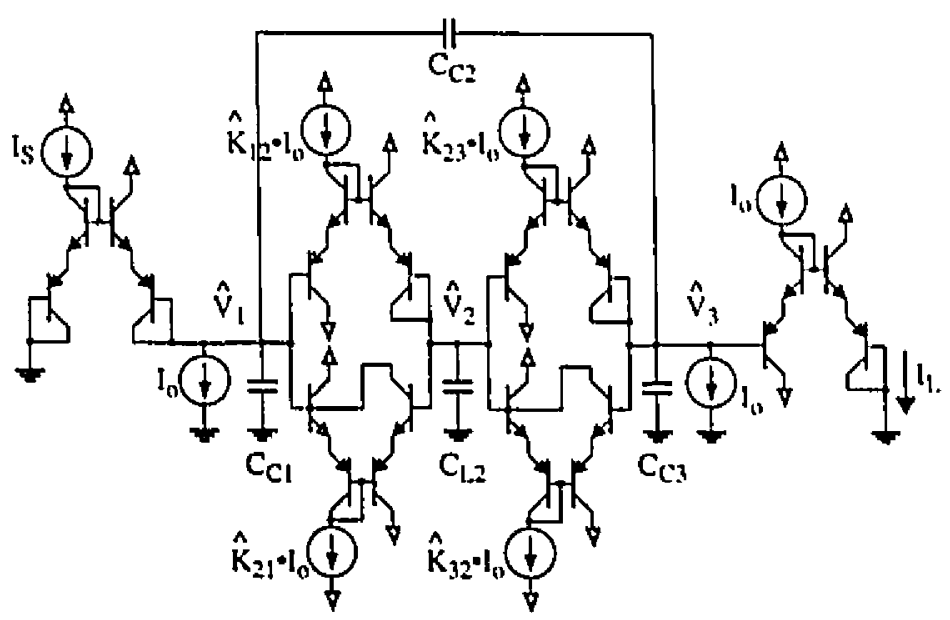

Figure 4-3: Circuit diagram for a 3rd-order elliptic log-domain filter

Multiplying through by $e^{\hat{v}_{3} / 2 V_{T}}$ gives:

$$
C_{C 3} \cdot e^{\hat{V}_{3} / 2 V_{T} d} \frac{d}{d t} \hat{V}_{3}=\hat{K}_{23} \cdot I_{0} e^{\hat{V}_{2} / 2 V_{T}}+\ldots+C_{C 2} \cdot e^{\hat{V}_{3} / 2 V_{T} d} \frac{d}{d t} \hat{V}_{1}-C_{C 2} \cdot e^{\hat{V}_{3} / 2 V_{T} d} \frac{\hat{V}_{3}}{d t}
$$

We must now make the following approximation in order to establish a correspondence between the elliptic filter circuit and the signal-flow graph derived from the LC ladder:

$$
e^{\hat{V}_{1} / 2 V_{T}}=e^{\hat{V}_{3} / 2 V_{T}}
$$

While such an assumption is not immediately obvious, practical experience with the logdomain elliptic filter has proven it to be valid. This allows us to rewrite Eq. (4.3) as:

$$
C_{C 3} \cdot e^{\hat{v}_{3} / 2 V_{T} d} \frac{d}{d t} \hat{V}_{3}=\hat{K}_{23} \cdot I_{o} e^{\hat{v}_{2} / 2 V_{T}}+\ldots+C_{C 2} \cdot e^{\hat{v}_{1} / 2 v_{r} d} \frac{\hat{V}_{1}}{d t}-C_{C 2} \cdot e^{\hat{v}_{3} / 2 v_{r} d} \frac{\hat{V}_{3}}{d t}
$$

Applying the chain rule gives:

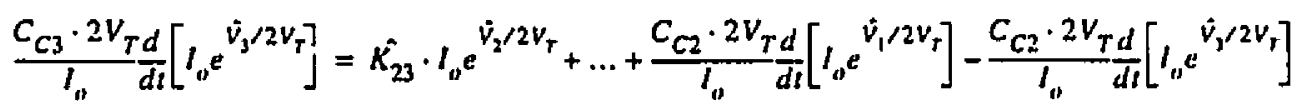

The factor $I_{o} / 2 V_{T}$ can be absorbed into the constant $\hat{K_{23}}$ such that: 


$$
K_{23}=\frac{\hat{K_{23}} \cdot I_{o}}{2 V_{T}}
$$

Which results in the equation:

$$
C_{C 3} \frac{d}{d t}\left[I_{0} e^{\hat{V}_{3} / 2 V_{T}}\right]=K_{23} I_{0} e^{\hat{V}_{2} / 2 V_{T}}+\ldots+C_{C 2} \frac{d}{d t}\left[I_{0} e^{\hat{V}_{1} / 2 V_{T}}\right]-C_{C 2} \frac{d}{d t}\left[I_{0} e^{\hat{V}_{3} / 2 V_{T}}\right]
$$

This equation can be rewritten in terms of the $L O G$ and $A N T I-L O G$ functions defined in Chapter 2, as:

$$
C_{C 3} \cdot \frac{d}{d t}\left[\operatorname{ANTI}\left(\hat{v}_{3}\right)\right]=\kappa_{23} \cdot \operatorname{ANTl}\left(\hat{v}_{2}\right)+\ldots+C_{C 2} \cdot \frac{d}{d t}\left[\operatorname{ANTl}\left(\hat{v}_{1}\right)\right]-C_{C 2} \cdot \frac{d}{d t}\left[\operatorname{ANTI}\left(\hat{v}_{3}\right)\right]
$$

Rewriting Eq. (4.8) and simplifying leads to the final form of the equation shown below:

$$
\hat{V}_{3}=\operatorname{LOG}\left\{\frac{1}{C_{C 3}} \int\left\{A N T I\left(\hat{V}_{2}\right)+\ldots+C_{C 2} \cdot \frac{d}{d t}\left[A N T I\left(\hat{V}_{1}\right)-A N T I\left(\hat{V}_{3}\right)\right]\right\}\right\}
$$

A comparison between Eq. (4.9) and the signal-flow graph of Figure 4-2(b) shows that we do indeed have a circuit which will implement the transfer function of an elliptic filter (assuming of course that the approximation of Eq. (4.3) is valid). Simulation results will be shown next.

\subsubsection{Simulation Results}

As with the Chebyshev log-domain filter, the frequency performance of this filter will be tested using both a large-signal form of HSPICE analysis (multitone analysis) and HSPICE small-signal analysis (AC analysis). This ensures that the log-domain filter will perform properly for all possible levels of input current.

The results of the HSPICE frequency analysis are shown in Figure 4-4. Figure 44(a) shows multitone testing while Figures 4-4(b) and 4-4(c) give the results of the AC analysis. As we saw with the Chebyshev filter we get excellent performance when ideal transistors are used such that the filter specifications are exactly met. There is a slight shift in the cutoff frequency and the shape of the passband ripple when Gennum transistors are 


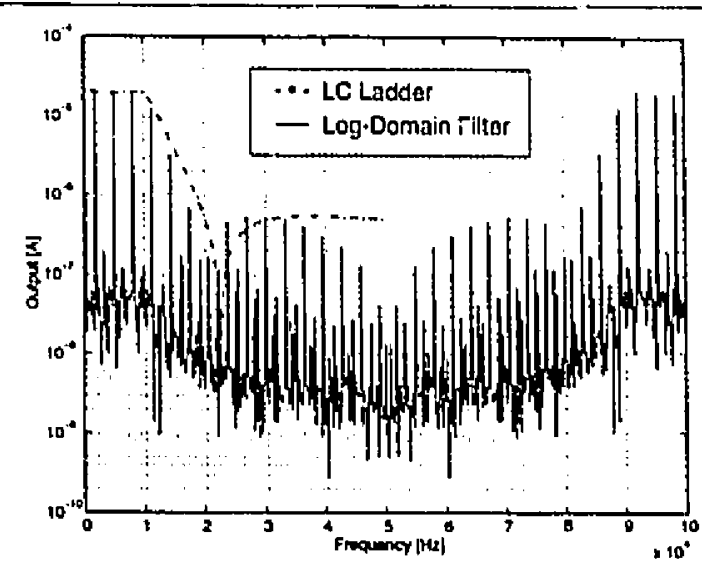

(a)

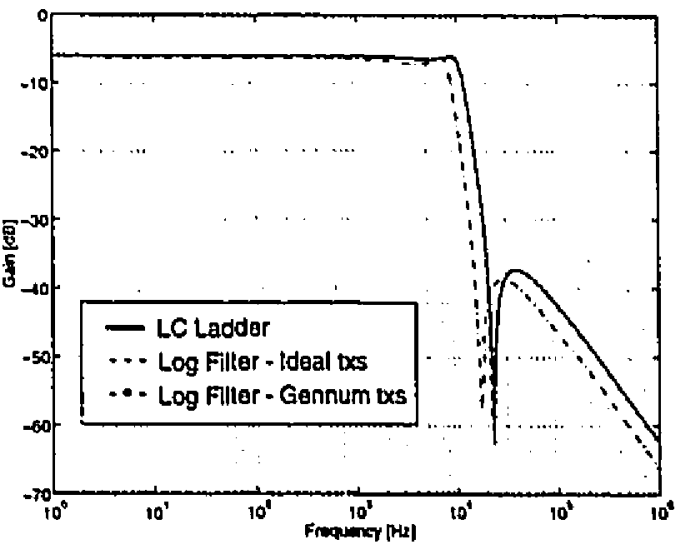

(b)

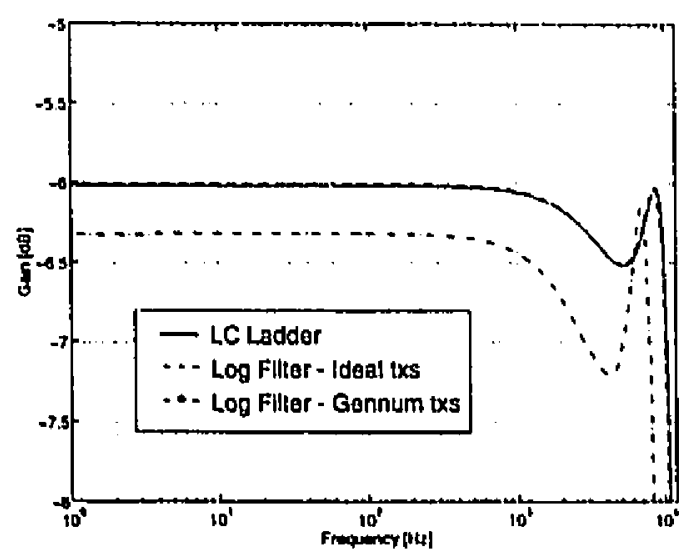

(c)

Figure 4-4: Frequency analysis of the log-domain elliptic filter (a) Multitone anaiysis (b) AC analysis (full scale) (c) AC analysis (passband)

substituted. This is again primarily due to the finite $\beta$ of the Gennum transistors which gives rise to base current loss and has a noticeable effect on the performance of the filter. There is little evidence that the approximation that was used when deriving the elliptic log-domain filter had an adverse effect on its frequency response. This will be further confirmed by the experimental results of Chapter 5 .

\subsection{Bandpass Filters}

\subsubsection{Derivation of a Fourth-Order Log-Domain Bandpass Filter}

The first step in the design of a bandpass filter is to find an LC ladder with the 


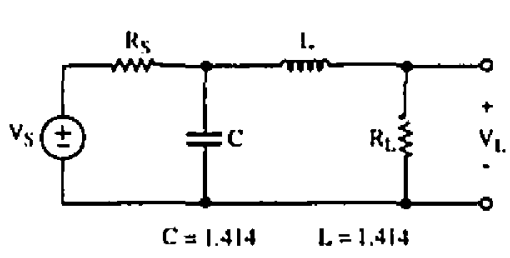

(a)

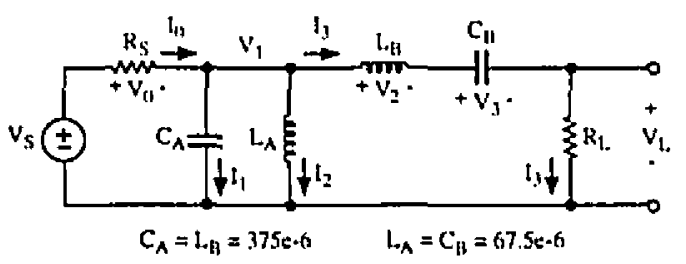

(b)

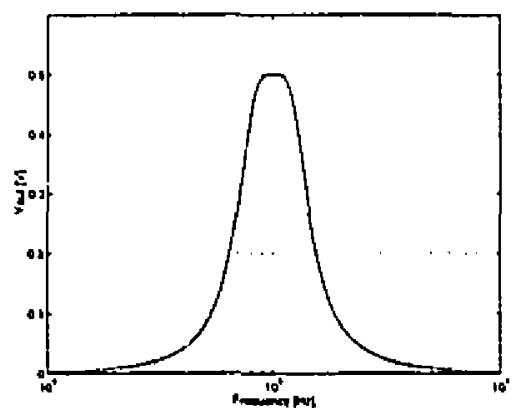

(c)

Figure 4-5: (a) Lowpass Prototype (b) Bandpass LC ladder (c) Magnitude response of the bandpass LC ladder

desired specifications. The design process begins with the choice of a suitable lowpass prototype. As we wish to design a fourth-order bandpass filter we choose the second-order Butterworth filter shown in Figure 4-5(a) as the lowpass prototype. Applying the appropriate LC circuit transformations [23] leads to the fourth-order bandpass filter shown in Figure 4-5(b). The filter was designed such that it has a cutoff frequency of $1 \mathrm{kHz}$ and a $600 \mathrm{~Hz}$ bandwidth. Its magnitude response is shown in Figure 4-5(c).

The next step is to represent the LC ladder by its signal-flow graph. Because of the limited set of building blocks available, we must ensure that the signal-flow graph contains only integrators along with summing and multiplication operations. The ladder of Figure 4-5(b) would then be represented by the following set of equations (Laplace notation has been used to save space):

$$
I_{1}=\frac{V_{S}-V_{1}}{R_{S}}-I_{2}-I_{3}
$$




$$
\begin{gathered}
V_{1}=\frac{1}{C_{A} s} I_{1} \\
I_{2}=\frac{1}{L_{A} s} V_{1} \\
V_{2}=V_{1}-V_{3}-R_{L} I_{3} \\
I_{3}=\frac{1}{L_{B} s} V_{2} \\
V_{3}=\frac{1}{C_{B} s} I_{3} \\
V_{L}=R_{L} \cdot I_{3}
\end{gathered}
$$

These equations result in the signal-flow graph of Figure 4-6(a).

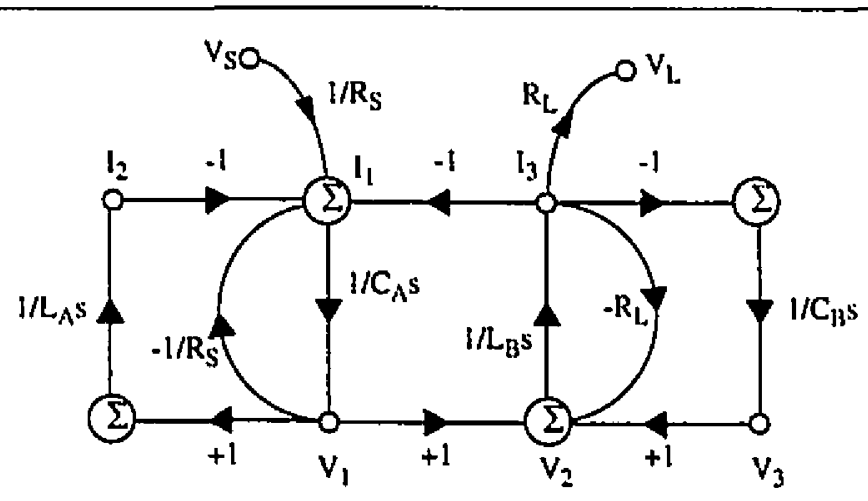

(a)

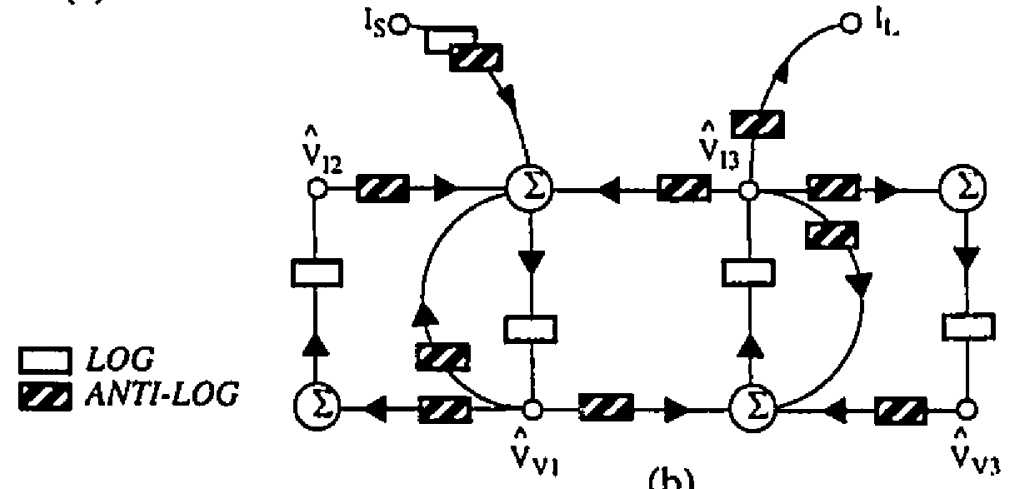

(b)

Figure 4-6: (a) SFG for the 4th-order bandpass filter (b) Equivalent log-domain SFG 


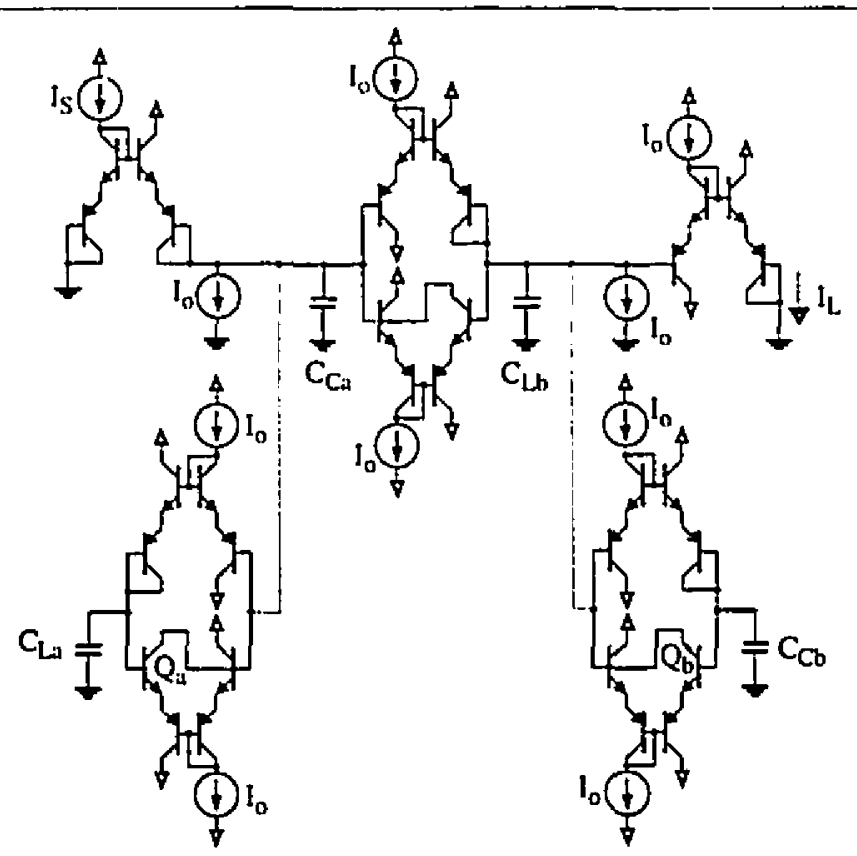

Figure 4-7: A 4th-order log-domain bandpass filter

In order to transform this signal-flow graph into the desired log-domain form we apply the rules of Section 3.2. This results in the new signal-flow graph shown in Figure 46 (b). Note that multiplier coefficients in the log-domain signal-flow graph are the same as those in the original signal-flow graph but have been omitted for the sake of clarity. The building blocks of Chapter 2 are then used to create the log-domain circuit for a bandpass filter (Figure 4-7).

\subsubsection{Simulation Results}

Figure 4-8 shows HSPICE simulation results for the bandpass log-domain filter derived previously. As always, the analysis was performed using both the multitone approach described in Appendix $\mathrm{A}$ and $\mathrm{AC}$ analysis. The response of the bandpass filter did not meet the specifications as well as for the lowpass log-domain filters. Although the filter performs a general bandpass operation it falls short of the specifications in terms of cutoff frequency, gain and ripple behavior. These can be compensated for by changing the bias currents which can be used to scale both the gain and the cutoff frequency. More 


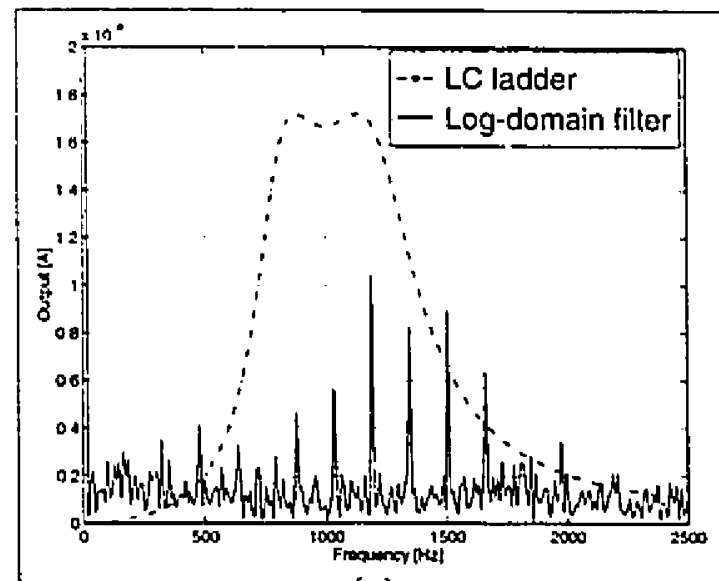

(a)

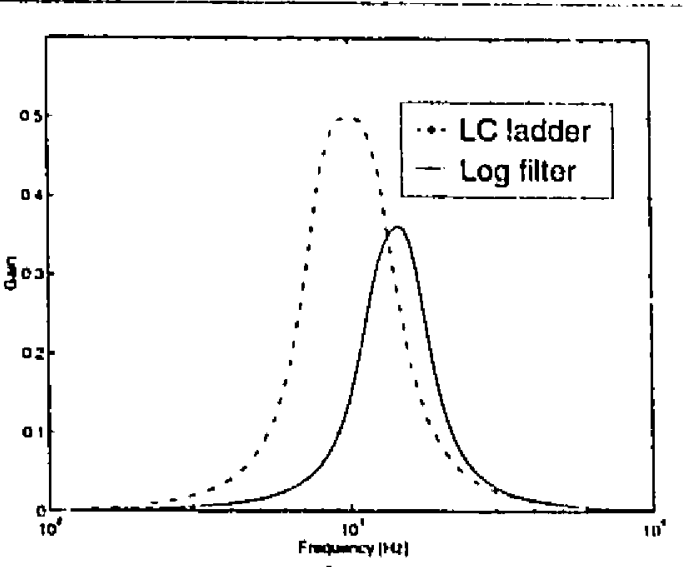

(b)

Figure 4-8: Simulated frequency response of the bandpass logdomain filter (a) Multitone analysis (b) AC onalysis

importantly however, ihe transistors labelled $Q_{a}$ and $Q_{b}$ tend to saturate under large signal conditions. This is due to the fact that there is no DC path to ground for the current flowing into $\mathrm{C}_{\mathrm{La}}$ and $\mathrm{C}_{\mathrm{Cb}}$. Future work in this area could explore the development of more stable structures for bandpass filtering.

\subsection{Summary}

In this chapter, the general method for the design of log-domain filters was used to design a third-order elliptic filter and a fourth-order bandpass one. By using an approximation, we were able to implement the elliptic filter by simply adding a series capacitor to the Chebyshev design found previously. HSPICE simulation confirmed that this approximation was acceptable. HSPICE was also used to simulate the bandpass filter which showed the required frequency behavior for small input levels but which ran into a problem with transistor saturation for larger inputs. 


\section{CHAPTER 5 Experimental Results}

\subsection{The Biquadratic Filter}

The first circuit whose experimental performance will be described is the logdomain biquadratic filter. This particular circuit was first introduced by Frey in [5] although the same circuit can be found using the design method introduced in this thesis. The complete circuit is shown in Figure 5-1. These results are of particular importance since they represent the first experimental confirmation of the log-domain filtering technique [33]. This section begins with a description of the experimental set-up used for

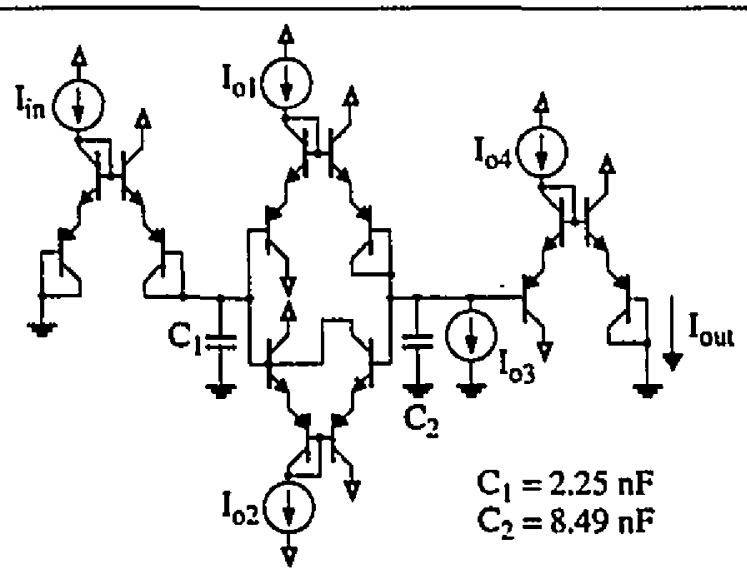

Figure 5-1: The log-domain biquadratic filter 
testing the biquadratic filter. The experimental results wilt then fall into two main categories (much as they did in the simulation results), namely frequency performance and linearity measurements. In the first, we compare the actual response of the biyuad to its intended transfer function, then show tunability and high-frequency operation. We tixen present a series of linearity measurements, including total harmonic distortion, intermodulation distortion and signal-to-noise ratio.

\subsubsection{The Test Set-Up}

An integrated circuit of the log-domain biquadratic filter was fabricated using Gennum GA911 analog arrays [31]. These are semi-custom bipolar arrays that are conposed of fixed components which can be interconnected in whatever configuration the designer chooses. In other words, the designer has control over a single metal layer which is deposited over a number of fixed components. The Gennum process provides NPN and PNP transistors with $f_{T}$ s of $2.5 \mathrm{GHz}$ and $10 \mathrm{MHz}$ respectively. A microphotograph of the chip is shown in Figure 5-2.

The current sources in the circuit were built using a modified Wilson design. The circuit was biased with supplies of \pm 5 voits. In order to generate and measure the inpul and output currents, simple $\mathrm{V} / \mathrm{I}$ and $\mathrm{I} / \mathrm{V}$ converters were included in the test circuit (sec Figure 5-3). The input was generated on an HP3314A function generator while the frequency analysis was performed using the HP3588 $\rfloor$ spectrum analyzer.

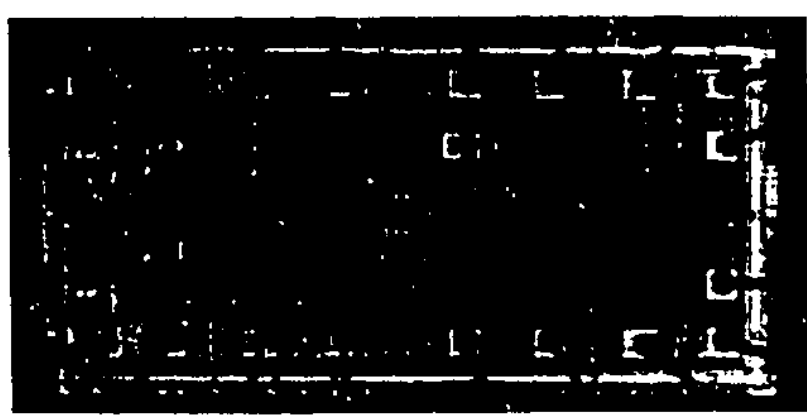

Figure 5-2: Microphotograph of the log-domain biquad 


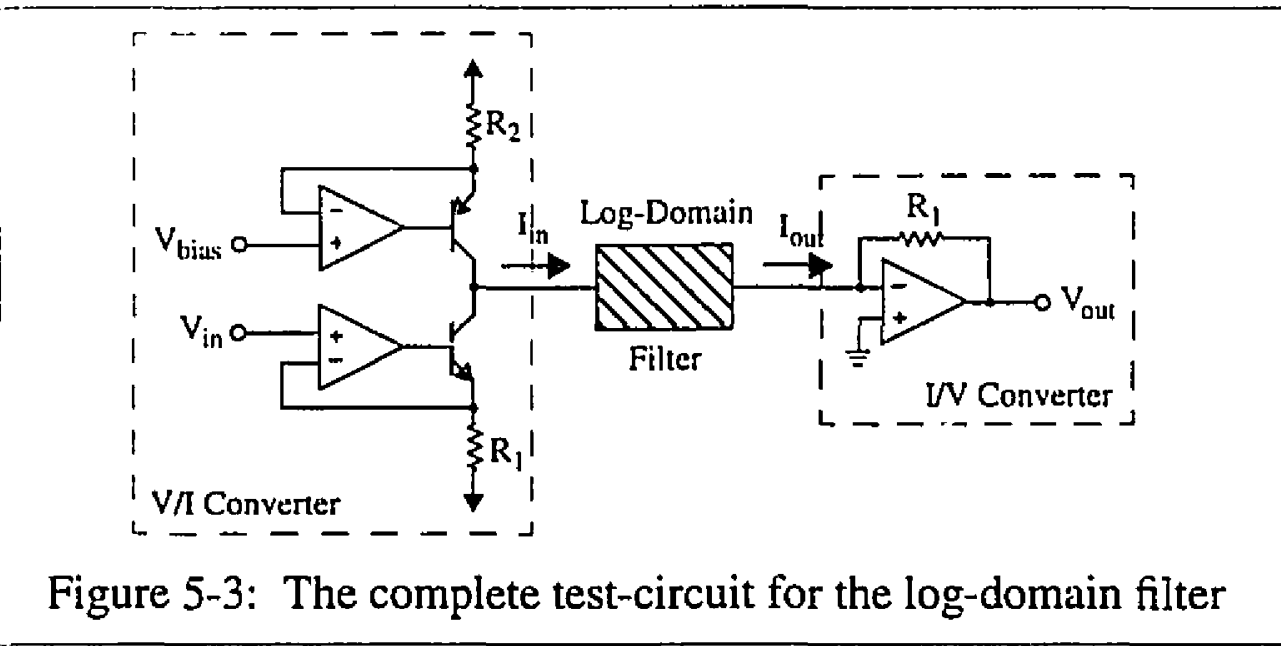

\subsubsection{Frequency Perforinance}

The magnitude response of the filter was first found and plotted versus the second-order transfer function that it was designed to implement. The results are shown in Figure 5-4. The experimental response was found by applying a peak-to-peak current input of $100 \mu \mathrm{A}$ and sweeping it from $10 \mathrm{~Hz}$ to $1 \mathrm{MHz}$. As is evident from the results, the biquad has the expected transfer function. The cutoff frequency is correct within a small experimental error, due to the finite $\beta$ of the transistors.

\subsubsection{Tunability}

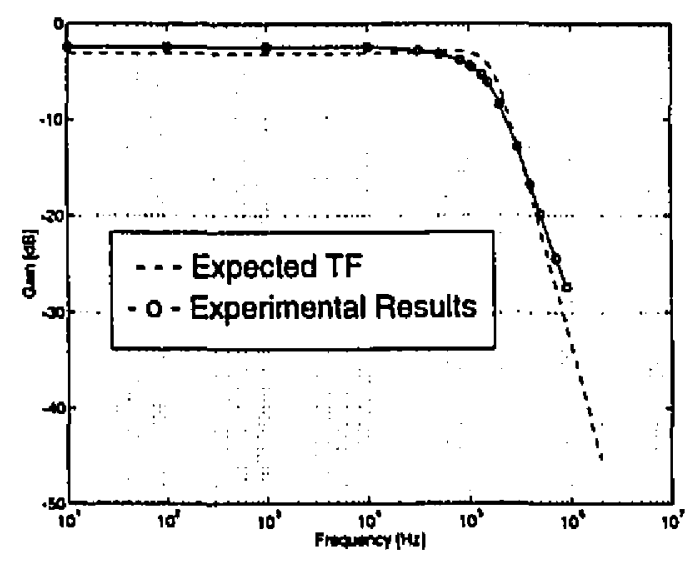

Figure 5-4: Frequency response of the biquad 
Another advantage of the log-domain filter is its tunability, i.e. the case in which the cutoff frequency of the circuit can be varied. The tunability of the log lilter can be attributed to the factor $J_{0} / 2 V_{T}$ which must be accounted for when using a log-domain integrator instead of a traditional integrator. This factor is implemented by either varying the capacitors or the bias currents in the circuit. This theory was tested experimentally by varying all of the bias currents in the circuit. The filter showed lunability over Iwo decades, namely from $1 \mathrm{kHz}$ to $100 \mathrm{kHz}$. A plot of the magnitude response of the biquadratic filter for three different levels of bias current is shown in Figure 5-5(a).

\subsubsection{High-frequency operation}

The final frequency test which was performed on the biquadratic filler was to measure how close the cutoff frequency could be brought to the $f_{t}$ of the slowest transistor (10 $\mathrm{MHz}$ for the GA911 Gennum process). To do so, the capacitors in the circuit were varied such that the cutoff frequency gradually increased. Figure 5-5(b) shows plots of the magnitude response of the filter for four different cutoff frequencies: $50 \mathrm{kHz}, 500 \mathrm{kHz}, 1$ $\mathrm{MHz}$ and $5 \mathrm{MHz}$. From these results we can see that the passband remains flat up to a cutoff frequency of $1 \mathrm{MHz}$ or $1 / 10$ th of the $f_{t}$ of the slowest transistor.

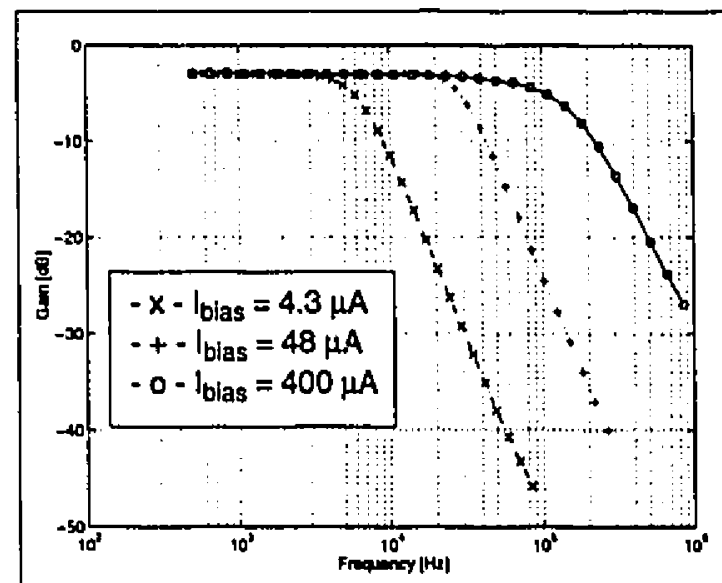

(a)

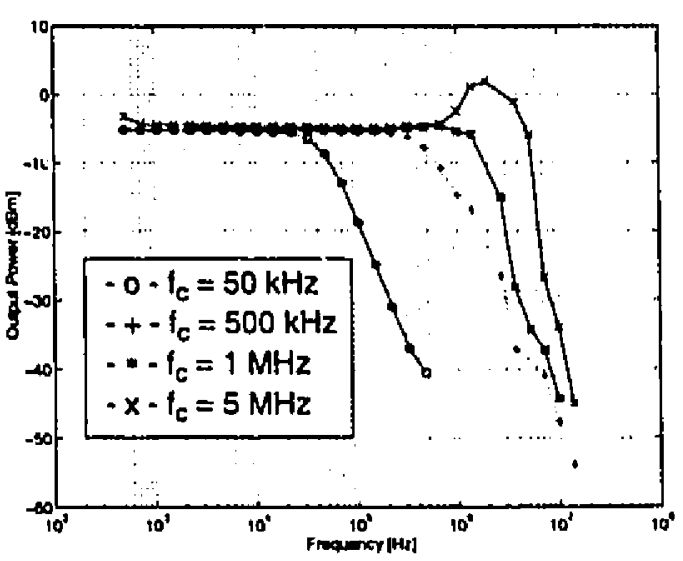

(b)

Figure 5-5: (a) Tunability of the log-domain biquad (b) The magnitude response of the biquad for 4 different cutoff frequencies 


\subsubsection{Linearity Measurements}

\subsubsection{Total Harmonic Distortion}

One of the most common measures of linearity is total harmonic distortion (THD). This is a measure of the total power of the harmonics to the power of the fundamental. By applying a test signal to the circuit and observing the output on a spectrum analyzer we can measure the harmonics and thus the total harmonic distortion. A spectral plot of the output for the circuit stimulated by a $100 \mu \mathrm{A}, 1 \mathrm{kHz}$ sine wave is shown in Figure 5-6(a). Given a spectral plot of this kind, the total harmonic distortion can be calculated using Eq. (3.8). Table 5-1 shows THD measurements for a range of different input amplitudes and frequencies. All measurements were taken over a $50 \mathrm{kHz}$ bandwidth. The biquadratic log-domain filter consistently showed distortion levels of less than -60 $\mathrm{dB}$.

\begin{tabular}{|l|l|l|l|}
\hline \multicolumn{1}{|c|}{$I_{\text {in }}$} & Frequency & \multicolumn{2}{c|}{ THD } \\
\hline \hline $100 \mu \mathrm{A}$ & $1 \mathrm{kHz}$ & $-62.4 \mathrm{~dB}$ & $0.071 \%$ \\
\hline $10 \mu \mathrm{A}$ & $1 \mathrm{kHz}$ & $-62.9 \mathrm{~dB}$ & $0.076 \%$ \\
\hline $100 \mu \mathrm{A}$ & $5 \mathrm{kHz}$ & $-60.3 \mathrm{~dB}$ & $0.096 \%$ \\
\hline
\end{tabular}

Table 5-1: THD measurements for the log-domain biquad

\subsubsection{Intermodulation Distortion}

One of the disadvantages of THD as a measure of linearity is that the harmonics may be affected by the filtering characteristics of the device under test. This is particularly relevant when dealing with lowpass or bandpass filters. A better test of linearity in such cases is the measurement of intermodulation distortion (IMD) described in Section 3.4.2.3.

A spectral plot of the log-domain biquad stimulated by two tones of slightly less 
Chapter 5: Experimental Re'sults

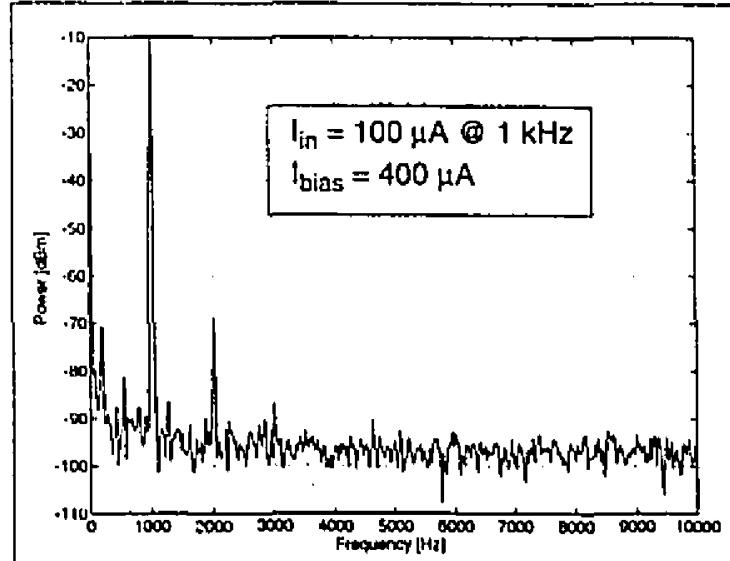

(a)

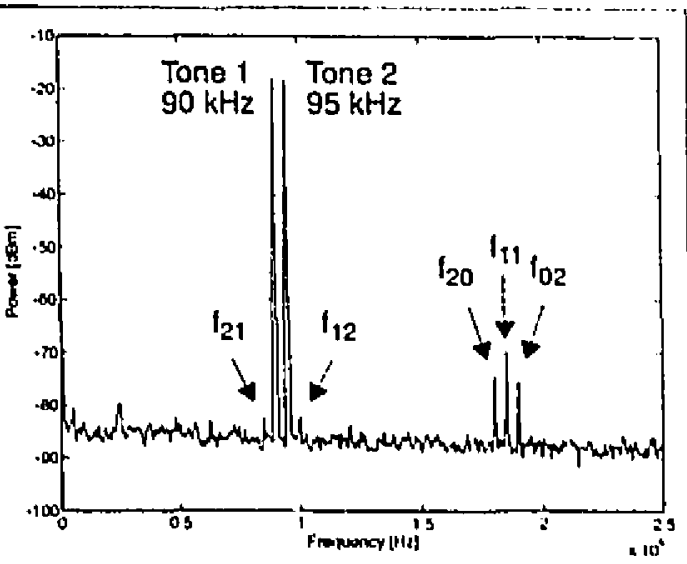

(b)

Figure 5-6: Spectral response of the biquad (a) Stimulated by a single tone (b) Stimulated by two tones

than the cutoff frequency of the filter is shown in Figure 5-6(a). The components $\int_{20}$ and $f_{02}$ represent the second-order distortion products which accounted for most of the total harmonic distortion found in the previous section. The tone $f_{I /}$ represents the sum of the two tones and is traditionally $6 \mathrm{~dB}$ greater than the second order components, as is the case here. We concentrate here on the distortion components close to the original tones $\left(f_{12}\right.$ and $\left.f_{2 l}\right)$ since these fall near the desired frequencies and can prove the most troublesome [32]. In Figure 5-7(a), we show a plot of the sum of the power of these thirdorder harmonics versus the amplitude of one of the input tones. The second tone was of equal amplitude. Also included on the graph is a plot of the power of the sum of the two fundamentals versus input current. The least intermodulation distortion occurs for an input of $100 \mu \mathrm{A}$ and measures $-70 \mathrm{~dB}$. The IMD then increases due to the cubic relationship between these harmonics and the input signal. At low input levels, the power of the harmonics remains constant due to the fact that they are so small they get lost below the noise floor.

\subsubsection{Signal-to-Noise Ratio}

The final test performed on the biquad was to find the signal-to-noise-plus- 


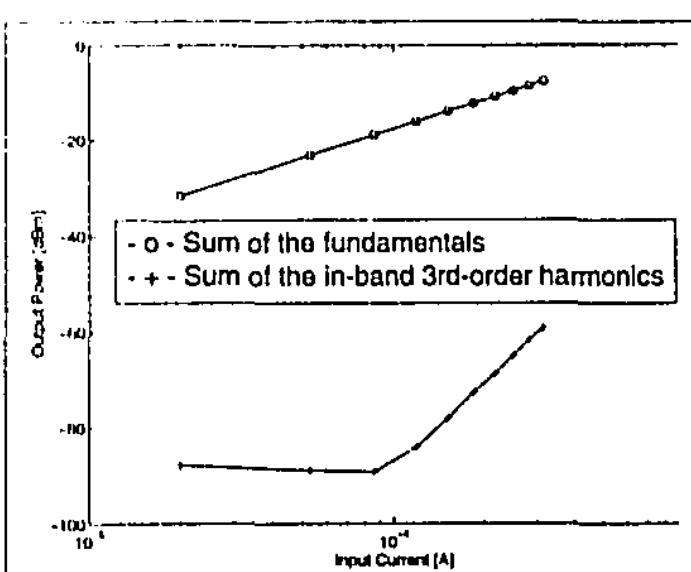

(a)

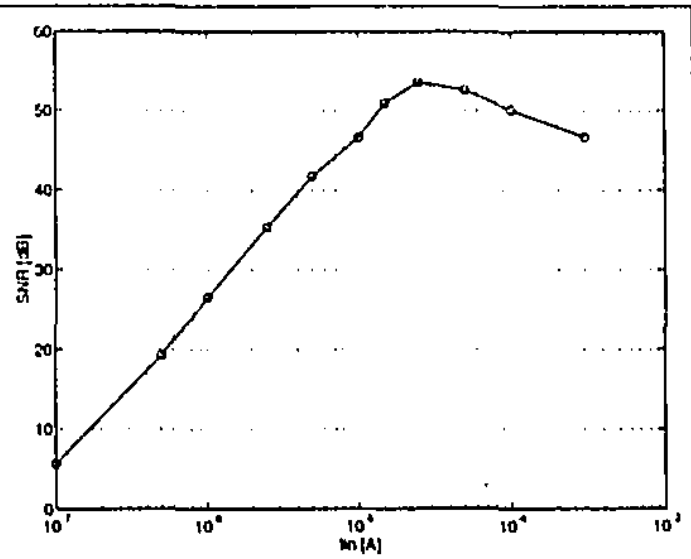

(b)

Figure 5-7: (a) IMD vs. input current (b) SNR vs. input current (measured over a $50 \mathrm{kHz} \mathrm{BW}$ )

distortion ratio (SNR) for different levels of input current. The performance of the biquad biased with $400 \mu \mathrm{A}$ current sources is shown in Figure 5-7(b). The measurement was made over a $50 \mathrm{kHz}$ bandwidth. The signal-to-noise ratio increases linearly up to a peakto-peak signal of $25 \mu \mathrm{A}$ then we see that the harmonics begin to dominate. The maximum attainable signal-to-noise ratio with this circuit was $54 \mathrm{~dB}$. From these results together with those of the previous sections we see that the distortion behavior of the biquadratic filter is best between 10 and $100 \mu \mathrm{A}$. This is consistent with the transistor specifications which shows that the transistors have their highest $\beta$ levels for currents in that range.

\subsection{The Fifth-Order Chebyshev Filter}

In order to confirm the design method presented in this thesis, a fifth-order Chebyshev log-domain filter like the one proposed in Chapter 3 was fabricated using the Gennum bipolar process presented earlier. In this section, we will show experimental results which verify the performance of this filter. Once again, we focus on the frequency response and linearity of these circuits since these best allow us to judge the usefulness of these new techniques. 


\subsubsection{The Test Set-up}

The fifth-order Chebyshev filter was fabricated with a larger version of the Gennum GA911 bipolar array used for the biquadratic filter. As with the biquad, modilied Wilson current mirrors were used for the current sources and the circuit was biatsed at \pm 5 volts. All of the external test circuitry and equipment was the same as described previously.

\subsubsection{Frequency Performance}

The components of the Chebyshev filter were chosen such that it had a cutofl frequency of $50 \mathrm{kHz}$ and a $1 \mathrm{~dB}$ ripple. A plot of the spectral response of the filter is shown in Figure 5-8(a) along with a close-up of the passband (Figure 5-8(b)). The tigure also shows a plot of the frequency response of the LC ladder which met the original specifications along with HSPICE AC analysis of the filter modelled using both ideal and Gennum transistors.

The results show that the fifth-order log-domain filter has very much the desired frequency response. Most importantly, we see the desired rippling behavior in the passband and the correct attenuation in the transition and stopband regions (until the noise

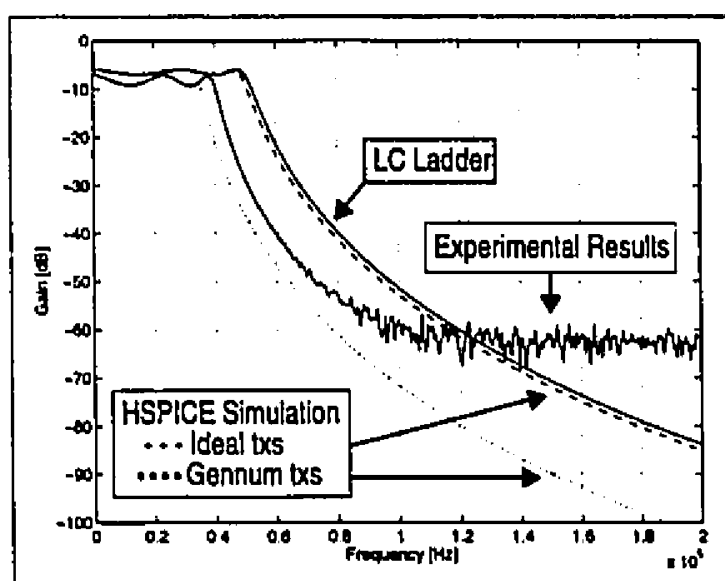

(a)

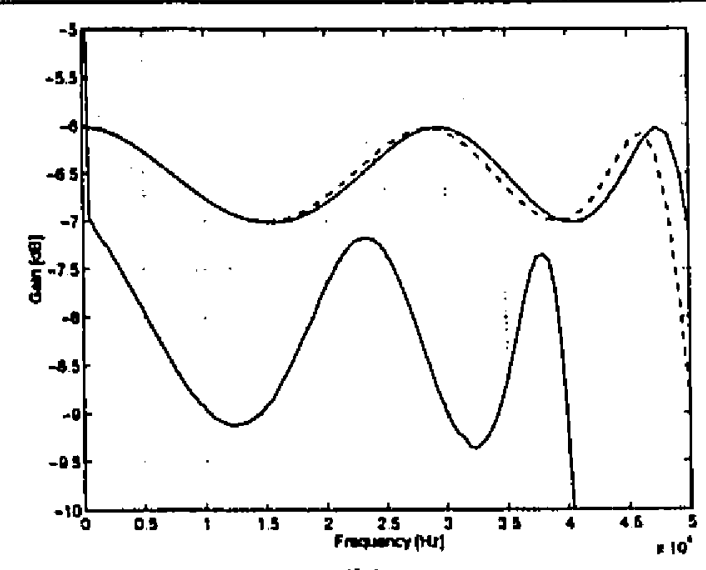

(b)

Figure 5-8: (a) Magnitude response of the 5th-order Chebyshev $\log$ filter (b) Close-up of the passband 


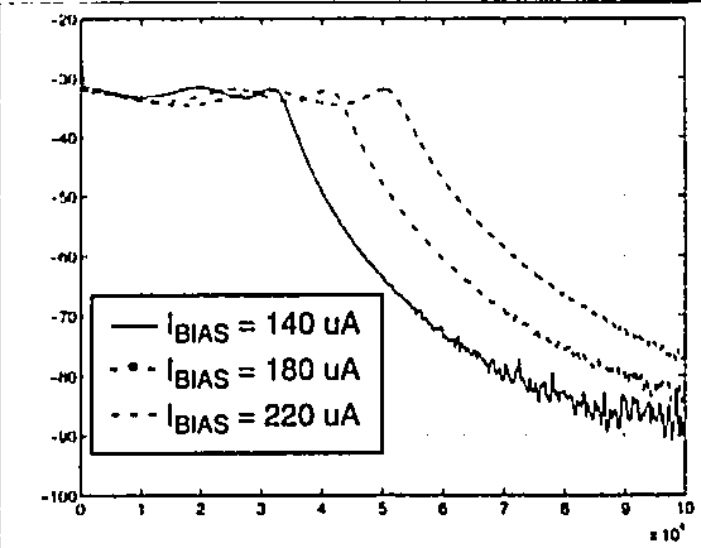

(a)

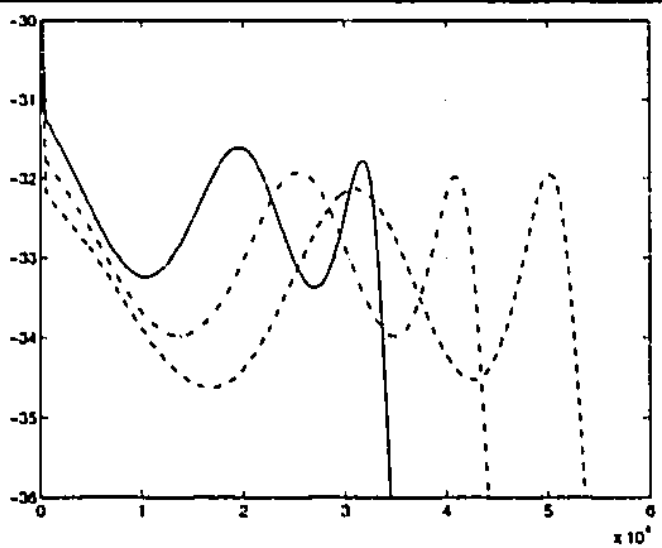

(b)

Figure 5-9: (a) Tunability of the 5th-order log filter (a) Full frequency range (b) Passband

floor is reached). There is a shift in the cutoff frequency by approximately $10 \mathrm{kHz}$ which is due to the non-ideal nature of the transistors. This can be seen in the simulation results where we see similar behavior when more reaiistic transistor models are used. More specifically, it is due to the finite $\beta$ of the bipolar transistor which results in a certain amount of base current loss. Since the cutoff frequency of the log-domain filter is directly proportional to the bias current, this base current loss translates into a shift in the cutoff frequency. It can be accounted for by designing the bias current such that it compensates for this current loss.

The tunability of this filter is demonstrated in Figure 5-8. Although the resistors which set the bias current of the circuit were fixed, we could still vary the supply voltages and thus change the bias in this manner. The general circuit operation was maintained although the new supply levels did have a slight effect on the filter response, as will be discussed shortly. Figure 5-8 shows the fifth-order Chebyshev filter biased with $\pm 4 \mathrm{~V}, \pm$ $5 \mathrm{~V}$ and $\pm 6 \mathrm{~V}$ supplies which corresponded to bias currents of $140 \mu \mathrm{A}, 180 \mu \mathrm{A}$ and $220 \mu \mathrm{A}$ respectively. Clearly, we can see how the bias current controls the cutoff frequency of the circuit while maintaining most other filter characteristics. Note however, that the passband 
ripple increased slightly as the supply voltages were increased. This is duc to the Early effect which modifies the ideal exponential nature of the bipolar transistor. It becomes more pronounced when higher voltage supplies are used since it is related to the transistor voltage $\mathrm{V}_{\mathrm{CE}}$.

\subsubsection{Linearity Measurements}

\subsubsection{Total Hamonic Distortion}

Figure 5-10(a) shows a plot of the total harmonic distortion of the fifth-order Chebyshev log-domain filter versus input current. The input was a $2 \mathrm{kHz}$ sine wave of varying amplitude. The frequency of the input tone was chosen such that it was weil below the cutoff frequency of the filter so that the harmonics were not affected by the natural attenuation of the filter. The fact that the input frequency is below the passband edge leads to slightly better distortion measurements than might be found for an input signal placed right at the cutoff frequency. This is why the intermodulation distortion test is a better measure of linearity.

The best harmonic distortion measure was $-47 \mathrm{~dB}$ found for an input of $15 \mu \mathrm{A}$ and a bias current of $180 \mu \mathrm{A}$. The distortion is primarily due to the first harmonic found at

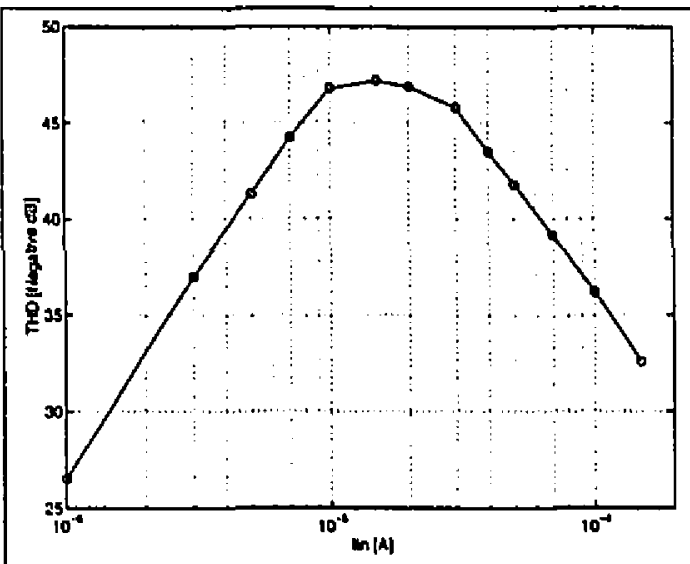

(a)

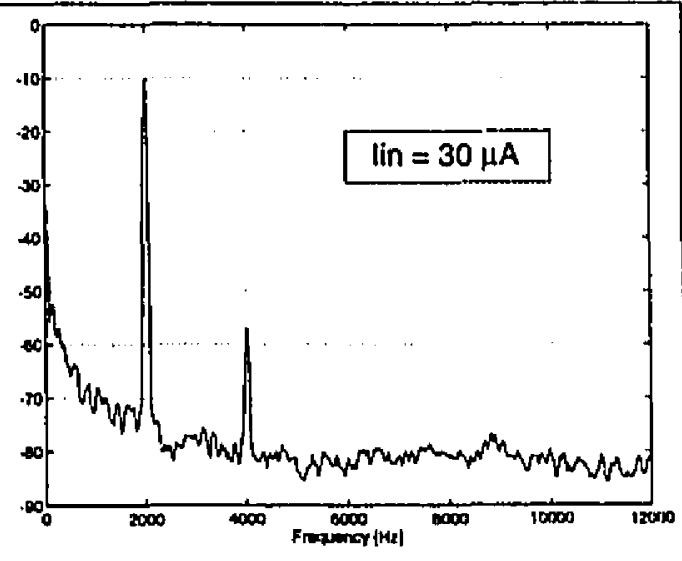

(b)

Figure 5-10: (a) THD of the log-domain Chebyshev filter vs. input current (b) Spectral plot of the filter output for a single tone input 
twice the frequency of the fundamental, as shown in a spectral plot of the filter stimulated by a $30 \mu \mathrm{A}, 2 \mathrm{kHz}$ sine wave (Figure $5-10(\mathrm{~b})$ ).

\subsubsection{Intermodulation Distortion}

In order to measure the intermodulation distortion (IMD) of the log-domain filter a two-tone stimulus was applied to the filter. The frequency of the two tones was chosen such they were $4 \mathrm{kHz}$ apart and they were placed slightly below the passband edge of the filter. As this is where the worse distortion occurs, this gives us a form of worst-case analysis of the linearity of the filter. Figure 5-11(b) shows the spectral response of the Chebyshev filter stimulated by tones of $28 \mathrm{kHz}$ and $32 \mathrm{kHz}$. One can clearly see the different harmonics and intermodulation products.

The products which are of the most interest to us are the third-order tones found at $24 \mathrm{kHz}$ and $36 \mathrm{kHz}$. Figure $5-11$ (a) shows a plot of the sum of the power of the fundamentals and of the sum of the power of the third-order harmonics versus input current. The difference between these two measurements gives us the intermodulation distortion. The best IMD measure for this filter was $-55 \mathrm{~dB}$ and occurred for an input of 30 $\mu \mathrm{A}$ and $180 \mu \mathrm{A}$ current sources.

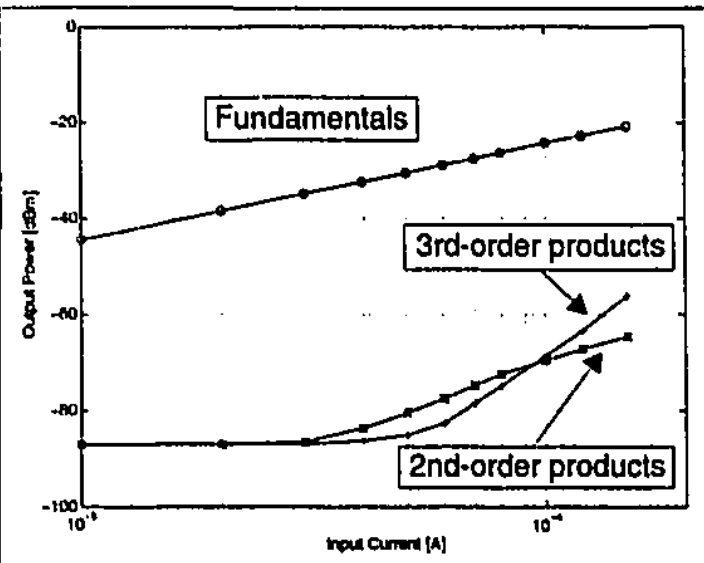

(a)

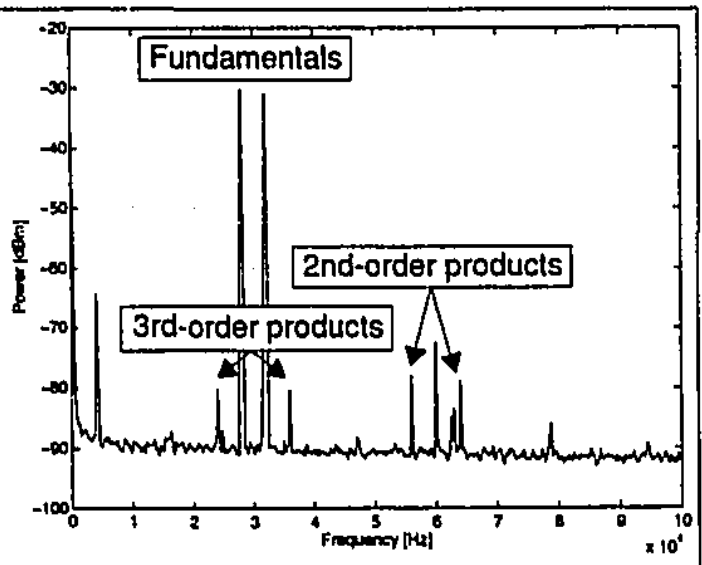

(b)

Figure 5-11: (a) IMD of the Chebyshev log filter vs. input current (b) Spectral plot of the filter output for a two tone input 
For comparison purposes, Figure 5-11(a) shows the sum of the power of the second-order products at $56 \mathrm{kHz}$ and $64 \mathrm{kHz}$ versus input current. The distortion measure found by evaluating these products is in effect the same as the THD measurement made in the previous section Unfortunately, the distortion may be affected by the fact that these products fall in the stopband region of the filter. Figure 5-11(a) also shows how the power of the third order products rises at a faster rate than the power of the second-order products. This is due to the cubic relationship between the third-order products and the signal.

\subsection{The Log-Domain Elliptic Filter}

A fifth-order log-domain elliptic filter was designed based on the techniques of Chapter 4 and then implemented in silicon using the bipolar design process described previously. This was easy to do since the circuit for a fifth-order elliptic filter is almost identical to that of a fifth-order Chebyshev filter. The only difference lies in the value of the existing capacitors and in the addition of two bypass capacitors.

The magnitude response of the elliptic log-domain filter is shown in Figure 5-12. The filter which was fabricated was based on an LC ladder with a cutoff frequency of 40 $\mathrm{kHz}$ and a $1 \mathrm{~dB}$ passband ripple (also shown in Figure 5-12). Upon examining the experimental response of the log-domain filter, we see that the basic response of an elliptic filter has been achieved. We can clearly see the presence of the finite transmission zeros which give rise to stopband ripple. The similarity between the experimental and expected results further confirms that the approximation made in Chapter 4 was valid (Eq. (4.3)).

As with the Chebyshev filter, the finite base current has given rise to a shift in the cutoff frequency and to degradation of the passband ripple. The HSPICE simulations included in Figure 5-12 show the effect of non-ideal transistors on the filter performance. Additional degradation of the passband may have been caused by variations in the 


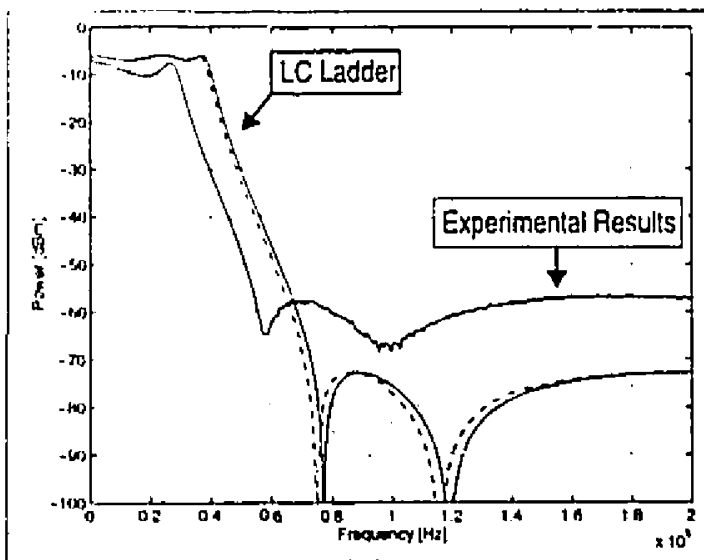

(a)

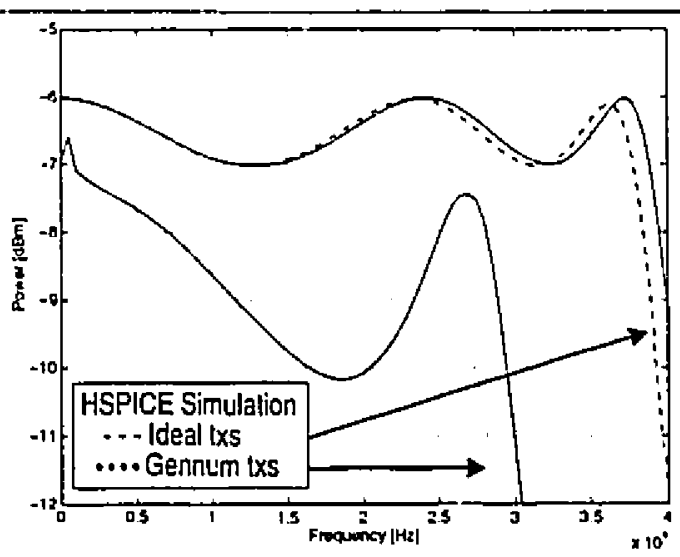

(b)

Figure 5-12: Frequency response of the elliptic log-domain filter (a) Full frequency scale (b) Passband

capacitor values.

The linearity of the log-domain elliptic filter was comparable to the results found for the fifth-order Chebyshev filter hence they will not be repeated here.

\subsection{Summary}

Experimentally, the log filters fabricated using the Gennum process gave similar results to those found through simulation (Chapters $3 \& 4$ ). This is a testament to the quality of the transistor models provided by Gennum.

Overall, the filters showed good correlation between their frequency response and the response required by the specifications. There was some degradation of the passband ripple and a slight shift in cutoff frequency caused by the base current loss of the bipolar transistors. The filters proved to be tunable over two decades and could be operated up to one tenth of the $f_{T}$ of the lowest transistor in the circuit. Distortion levels measured using a variety of methods ranged from $-45 \mathrm{~dB}$ to $-70 \mathrm{~dB}$. This is comparable to distortion levels found in many contin:sus-time filtering schemes [1]. 


\section{CHAPTER 6 Conclusion}

\subsection{Discussion of Results}

This thesis presented a novel technique for the design of log-domain filters based on the operational simulation of LC ladders. Because of the non-linear nature of logdomain integrators, the traditional operational simulation method was modified and a linearization procedure based on inverse functions was introduced. The strength of the LC ladder method is that it makes the design of high-order log-domain filters easier and provides the designer with greater insight into the circuit operation.

The tachnique was demonstrated through the step-by-step design of a fifth-order Chebyshev filter, as well as the design a third-order elliptic filter and a fourth-order bandpass filter. The behavior of these filters was verified through HSPICE simulation. A simulation procedure called multitone analysis was used to perform the initial verification of the filter's frequency behavior. It is based on HSPICE transient analysis and thus is valid for all input levels, large or small. This is as opposed to AC analysis which uses linearized small-signal models of the transistors thus negating their true exponential nature. The results showed good correlation betw en the simulated response and the 
original specifications. There was a slight diop in performance when real transistor models were used since the devices no longer behaved as perfect exponential functions. The only disturbing result was the presence of saturated transistors in the bandpass filter for larger input levels.

Log-domain filters were fabricated using a semi-custom bipolar design process provided by the Gennum Corporation. These results are of particular importance since they represent the first experimental results to be reported on this topic. As was the case in simulation, the filters showed excellent correlation between the experimental results and the desired specifications. There were some discrepancies which can be attributed to the non-ideality of the bipolar transistors. Most notably, the finite base current loss in the transistors gave rise to a shift in cutoff frequency of approximately $10 \mathrm{kHz}$. This can be compensated for since the filters can be tuned by adjusting the bias current. Experimentally, the filter showed tunability over at least two decades. The filters could be operated up to a frequency of $1 \mathrm{MHz}$ or $1 / 10$ th of the $f_{T}$ of the slowest transistor. In this respect, log-domain filtering can be classified as a fast filtering scheme.

Several distortion measurements were performed in order to verify the linearity of these filters. The harmonic distortion (THD) of these filters measured $-62 \mathrm{~dB}$ for the biquad and $-47 \mathrm{~dB}$ for the fifth-order Chebyshev. Intermodulation distortion (a more accepted distortion measurement as it can be performed with signals right at the passband edge) was $-70 \mathrm{~dB}$ for the biquad and $-55 \mathrm{~dB}$ for the Chebyshev filter.

In order to evaluate the performance of the filter in terms of our design goals a table has been prepared which compares the log-domain filter to some of the more recent papers on continuous-time filtering (Table 6-1). The design goals were high-frequency performance, tunability and low distortion. Overall, the log-domain filter shows - comparable performance to the other filters. While some of the filters shown may outperform the log filter in a specific area, it is usually at the expense of another property. 
In addition, the log-domain filter achieves these results using a circuit which is much simpler than the other proposed schemes.

\begin{tabular}{|c|c|c|c|c|c|c|}
\hline Author & Type & Order & Cutoft & $\sin _{\mathrm{T}}$ & Tunable & Distortion" \\
\hline Snelgrove \& Shoval [13] & $\mathrm{g}_{\mathrm{m}} \cdot \mathrm{C}$ & 2 & $450 \mathrm{MHz}$ & - & YES & $-4.5 \mathrm{~dB}$ (INID) \\
\hline B. Nauta [14] & $\mathrm{g}_{\mathrm{m}}-\mathrm{C}$ & 3 & $98 \mathrm{MHz}$. & - & YES & - \\
\hline Willingham \& Martin[17] & $\mathrm{g}_{m}-\mathrm{C}$ & 7 & $8 \mathrm{MHz}$. & $3 / 1000$ & NO & $-6510-80 \mathrm{~d}(3$ (THID) \\
\hline Moon \& Song [11] & MOS-C & 5 & $22 \mathrm{kHz}$ & - & NO & $-90 \mathrm{~dB}$ (IHID) \\
\hline Log-Domain Filter & Log & 2.5 & $1 \mathrm{MHz}$ & $1 / 10$ & YES & $-5510-70 \mathrm{~dB}(\mathrm{IMD})$ \\
\hline
\end{tabular}

$\dagger$. Direct comparison of distortion levels is difficult due to different experimental methods

Table 6-1: A comparison of continuous-time filters

\subsection{Topics of Future Research}

While this thesis has shown that the log-domain filter is a viable alternative to traditional continuous-time filtering methods, it is still a relatively new concept and much of its potential has yet to be explored.

High-Frequency Applications: In order to build log-domain filters operable at video rates two possibilities exist. First, the filters could be fabricated using a very good complementary bipolar process such that the filter is not limited by the poor highfrequency performance of the PNP transistors. Better yet would be the design of an all NPN log-domain filter. The technique described in this thesis could be used as long as all NPN versions of the log-domain integrators were derived.

Low-Voltage Design: The relatively simple nature of the log-domain filter along with the small voltage swings at the internal nodes due to the logarithmic compression of information makes this filtering method a good candidate for low-voltage circuit design.

Bandpass and Highpass Filters: The problem of transistor saturation in the bandpass 
filter for higher input levels needs to be addressed. A more stable design could perhaps be found based or an alternate signal-fiow graph implementation. The design of a highpass $\log$ filter has yet to be explored.

A MOSFET Implementation: The extension of the "log-domain" idea to MOS transistors would have the advantage of eliminating the error caused by base current loss and would make it more compatible with today's integrated circuits. The problem is not an easy one however since the MOS transistor does not behave according to an exponential function but rather according to a quadratic one. A similar theory would therefore have to be developed based on the quadratic function. 


\section{$\overline{\text { APPENDIX A }}$ Multitone Testing of the Log-Domain Filter}

HSPICE simulation is limited by its inability to perform AC analysis on nonlinear circuits. This is due to the fact that when performing $\mathrm{AC}$ analysis, HSPICE first solves for the DC operating point of the circuit, then determines linearized, small-signal models for all of the non-linear devices in the circuit. This is particularly rel:vant when testing the log-domain filter since the exponential nature of the bipolar transistor is at the very heart of its operation.

The solution is to use a technique called multitone testing [34]. Multitone testing involves applying a stimulus composed of one or more tones to the device under test and observing its spectral response. It is commonly used in the testing community and permits the simultaneous measurement of frequency response, total harmonic distortion (THD) and inter-modulation distortion (IMD).

This appendix will show how HSPICE transient analysis can be used to perform this kind of testing. The basic approach is as follows. First, HSPICE is used to find the transient response of a circuit subjected to a multitone stimulus. Then the mathematical 
software package MATLAB finds the discrete fourier transform of the transient output and piots it with respect to frequency. The design approaci; which is described in this appendix was used for all of the multitone analysis performed on the log-domain filters of this thesis.

\section{A.1 Review of the Discrete Fourier Transform}

In order to understand some of the constraints which will be imposed on the analysis, we begin with a review of the discrete Fourier transform (DFT) [35].

For every discrete-time sequence $x(n)$ there exists a Fourier transform which is defined by:

$$
X(\omega)=\sum_{-\infty}^{\infty} x(n) e^{-j \omega n}
$$

Suppose we now limit the length of $x(n)$ to L samples, such that:

$$
x(n)= \begin{cases}x(n) & 0 \leq n \leq L-1 \\ 0 & n>L-1\end{cases}
$$

The Fourier transform of this sequence will now be given by:

$$
X(\omega)=\sum_{n=0}^{L-1} x(n) e^{-j \omega n}
$$

We now sample $X(\omega)$ at $N$ equally spaced frequencies $\omega_{k}=\frac{2 \pi k}{N}, k=0,1, \ldots, N-1$ and $N \geq L$, such that:

$$
X(k) \equiv X\left(\frac{2 \pi k}{N}\right)=\sum_{n=0}^{L-1} x(n) e^{-j \frac{2 \pi k n}{N}}, k=0,1,2, \ldots, N-1
$$

which can be rewritten as: 


$$
X(k)=\sum_{n=0}^{N-1} x(n) e^{(-j 2 \pi k n) / N}, \quad k=0,1,2, \ldots N-1
$$

Eq. (A.3) denotes the relationship for transforming a sequence $\{x(n)\}$ of length $L \leq N$ into a sequence of frequency samples $\{X(k)\}$ of length $N$, and is called the discrete Eourier transform (DFT). The relationship which allows us to recover the sequence $\{x(n)\}$ from a set of frequency samples is called the inverse discrete Fourier transtorm (IDFT) and is given by:

$$
x(n)=\frac{1}{N} \cdot \sum_{k=0}^{N-1} X(k) e^{(j 2 \pi k n) / N}, \quad n=0,1,2, \ldots, N-1
$$

The DFT is usually computed using any of a number of efficient algorithms which are called Fast Fourier Transforms (FFT).

\section{A.2 The HSPICE File}

The first step in multitone analysis is to use HSPICE to find the transient response of a circuit stimulated by one or more sinusoidal tones. For those who are unfamiliar with transient analysis, HSPICE finds the circuit solution at a set of discrete-time intervals. As a result, the outcome of the analysis will be a sequence of discrete-time samples $\{x(n)\}$ which can be used to compute the discrete Fourier transform as described in the previous section.

The HSPICE file describes the different circuit components which make up the log-domain filter $[29,30]$. When preparing an $H S P I C E$ file for multitone analysis, special care must be taken in two particular areas. First, the signal sources which make up the multitone input must be properly defined such that they have the right frequency, amplitude and phase. Each of these areas will be discussed at some length. Second, the transient analysis requests must be chosen correctly since the computation of the FFT depends on the proper transient output. 


\section{A.2.1 The Signal sources}

The circuit is to be stimulated by a set of sinusoidal current sources each operating at a different frequency. This section will outline some guidelines which govern the choice of frequency, amplitude and phase of the different tones in the multitone input.

\section{A.2.I.I Frequency}

The choice of frequency for each different tone is important for two reasons:

1. The frequency of each input tone must correspond to one of the discrete frequency points in the DFT. This will ensure that every input tone completes an integral number of cycles over the total simulation time and thus prevents leakage and spreading effects.

2. The frequency of the different tones should not be multiples of one another. This will minimize the chance that their harmonics and intermodulation products coincide.

The choice of sumpling frequency and of the unit test period (a concept to be defined next) will help us meet these constraints:

\section{a) The unit test period}

In order to minimize leakage effects when calculating the DFT of a multitone system, we must ensure that each sinusoid in the multitone input completes an integral number periods over the time of the analysis. The shortest

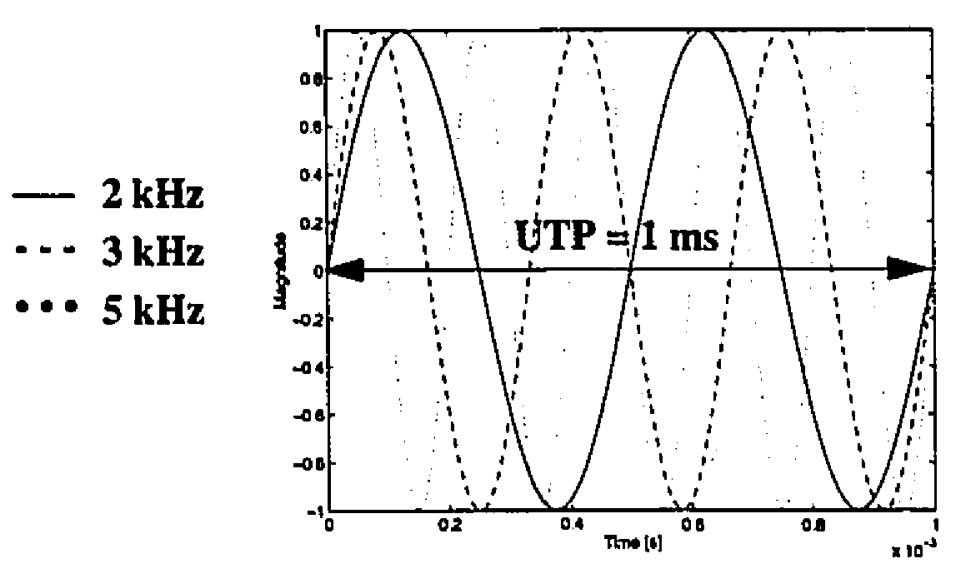

Figure A-1: Calculating the unit time period of a 3 tone input 
time interval which allows this for all tones is called the unit test periond (UTP). For example, the unit test period for an input composed of $2 \mathrm{kHz}, 3 \mathrm{kHz}$ and $5 \mathrm{kHz}$. sine waves is $I \mathrm{~ms}$, as shown in Figure A-1. The reciprocal of the UTP is called the primitive frequency and corresponds to the greatest divisor of the input frequencies ( $1 \mathrm{kHz}$ in our example). The value of the primitive frequency will hetp determine the sampling frequency.

b) The sampling frequency

Since we want each of the input frequencies to correspond to one of the discrete frequencies in the DFT, we let the sampling frequency be given by:

$$
f_{s}=N \cdot f_{p}
$$

where $N=$ Number of points in the $D F T$

$f_{p}=$ Primitive frequency

Figure A-2 shows the results of a 16 point DFT performed on a system composed of the three sine waves described in the previous section. Note that the sampling frequency is $16 \mathrm{kHz}$. Only the first eight samples are shown since the other eight are given by the mirror image of the first ones.

An additional problem which will affect the choice of the sampling frequency is aliasing. We know from the Nyquist Sampling Theory that in order to

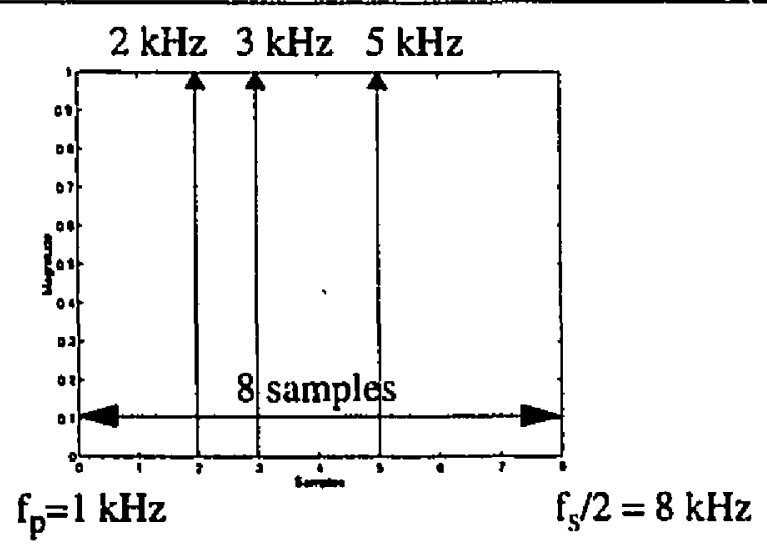

Figure A-2: Illustrating the relationship between the sampling frequency and other multitone parameters 
prevent aliasing, we must make sure that the highest frequency contained in the sampled signal is less than the Nyquist frequency, $f_{s} / 2$. In other words, all of the input tones must be at frequencies less than $f_{s} / 2$. Even with this precaution we may get aliasing if any of the harmonics or the inter-modulation products are greater than the Nyquist frequency. In this case, some kind of anti-aliasing filter would be needed. Most of the analysis done is this thesis was done such that the cutoff frequency of the filter to be tested was much less than $f_{s} / 2$. Therefore, the natural attenuation of the filter limits the effect of aliasing and eliminates the need for an anti-aliasing filter.

c) Frequency resolution

For a given sampling frequency, increasing the number of points in the DFT $(N)$ will increase the frequency resolution of the spectral output. Changing $N$ is analogous to controlling the resolution bandwidth on a spectrum analyzer. The drawback to choosing a large $N$ is that the number of samples needed for the transient analysis is large and thus increases the simulation time. Usually, one attempts to find a balance between spectral resolution and run time. A second constraint on $N$ is the type of FFT algorithm lised. A radix-2 algorithm needs $2^{N}$ samples while a radix-3 algorithm needs $3^{N}$ samples and so on. In our case, the radix-2 algorithm was always used since it is generally faster. As a result, $N$ must always be a power of 2 .

d) Interference

Each tone must be placed at a frequency which will minimize the chance that its harmonics and intermodulation distortion terms fall in the same frequency bin. This is in general a very complices d process. Often one simply tries to use a scheme which forces any possible overlapping harmonics to be of as high order as possible. There are a number of approaches which can be used:

\section{Prime-Rich Signals}


One strategy is to base the set of input frequencies exclusively on prime numbers. This ensures that none of the input frequencies are harmonic to one another and that no sum or difference products fall on a test frequency. Unfortunately, it does not prevent third and fith order intermodulation interference.

\section{An Iterative Scheme}

Restricting the tones to prime numbers is a tedious process. Because of the large number of constraints which are placed on the multitone stimulus we eventually want to be able to automate the process by writing a computer program which will automatically give us the frequency and other parameters for each tone. As a result, a formula for deriving the frequency of each tone was borrowed from telecom CODEC applications. The frequency of each tone is computed as follows:

$$
f_{\text {tone }_{i}}=\frac{M_{i}}{N} \cdot f_{s}
$$

where:

$$
\begin{aligned}
& f_{s}=\text { sampling frequency } \\
& N=\text { Number of points in the } D F T \\
& M_{i}=9+[i \times 16] \\
& i=0,1,2, \ldots \text { as long as } M_{i}<(N / 2)
\end{aligned}
$$

This method is more susceptible to interference between the harmonics of the different input tones than the prime-rich scheme was. For example, the frequency of the 16th harmonic of the first input tone will be the same as the frequency of the 16th tone; hence they will interfere with one another. The advantage of this particular scheme is that it is easier to implement algorithmically. The test engineer must decide whether the added interference is worth the greater ease of design. 


\section{A.2.1.2 Phase}

Because the periods of each sinusoid in the multitone input are related to one another, the sinusoids will tend to peak at the same points in :ime. When many tones are used this could lead to clipping in the circuit. The solution is to assign a random phase shift to each different sinusoid. This is easily done since $H S P I C E$ can accept a phase shift as one of the parameters of the input.

\section{A.2.1.3 Amplitude}

Even with phase shifting, the average power of the multitone signal will increase with the number of tones used. To prevent overdriving the circuit, the amplitude of each tone will be restricted to some reasonable value. Our strategy is to assign the amplitude of each sinusoid according to the following equation:

$$
\begin{aligned}
& \text { RMS of single tone }=\text { Desired } R M S \text { of multitone } / \sqrt{K} \\
& \text { where } K=\text { the number of tones in the multitone signal }
\end{aligned}
$$

For a sinusoid,

$$
\text { Peak of single tone }=\text { RMS of single tone } * \sqrt{2}
$$

Therefore,

$$
\text { Peak of single tone }=\text { Desired RMS of multitone } * \sqrt{\frac{2}{K}}
$$

\section{A.2.1.4 A complete HSPICE current source}

A typical $H S P I C E$ statement for one of the current sources in the multitone input is shown below:

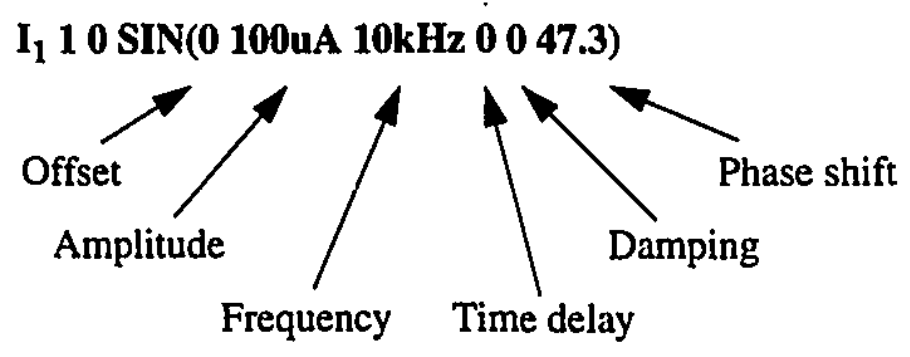


Some of these parameters are of no consequence in our amalysis and hence have been set to zero.

\section{A.2.2 Analysis Requests}

HSPICE is told what type of analysis to perform by a command called an analysis request. We wish to perform transient analysis such that we produce a sequence of discrete-time samples $\{x(n)\}$ which represent the output current of the log-domain filter. This sequence will then be used to compute its discrete Fourier transform

The HSPICE request for transient analysis is done through the .TRAN command. A typical .TRAN statement is shown below:

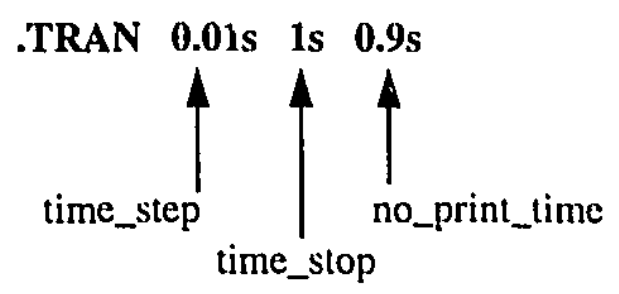

The three times are calculated as follows:

time_step: The step time is given by the sampling frequency.

$$
\text { time } e_{\text {step }}=\frac{1}{f_{s}}=T_{s}
$$

time_stop: The stop time would normally be the time for one unit test period which is related to the sampling frequency by:

$$
U T P=N \cdot T_{s}
$$

However, we wish to allow the simulation to run long enough for the output to settle down [36]. Therefore the total stop time is defined as:

$$
\text { time }_{\text {slop }}=N P \cdot N \cdot T_{s}
$$

where $N P=$ Number of periods needed for the simulation to settle down

no_print_time: This makes sure that only the last $\mathrm{N}$ points are printed, and is computed 
according to:

$$
\text { no_print_time }=\left[(N P-1) \cdot N \cdot T_{s}\right]+T_{s}
$$

We now have all the tools necessary to perform a spectral analysis of our circuit. The next section will show a simple example of how this would be done.

\section{A.3 An Example using the Log-Domain Biquad}

We wish to obtain the frequency response of the log-domain biquad described in Chapter 5. As we have some flexibility in choosing the component values of the bicluad, we will choose:

$$
\begin{aligned}
& l_{o}=100 u \mathrm{~A} \\
& C_{1}=8.2 n F \\
& C_{2}=2.2 n F
\end{aligned}
$$

which we know from theory should give us a cutoff frequency of:

$$
f_{c}=63.8 \mathrm{kHz}
$$

The sampling criteria is chosen as follows:

$$
\begin{gathered}
f_{\mathrm{s}}=500 \mathrm{kHz} \\
N=512 \text { points } \\
\text { Number of Tones }=16 \\
\text { Number of Periods }(\mathrm{NP})=100
\end{gathered}
$$

A C program was written which accounts for all of the criteria outlined in the previous two sections. A listing of the program can be found in Section A-4. The multitone inputs and the transient analysis statement were generated automatically and are shown below:

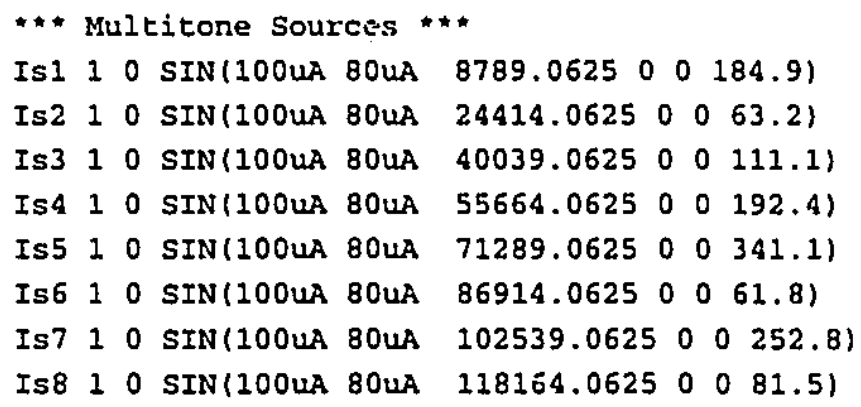




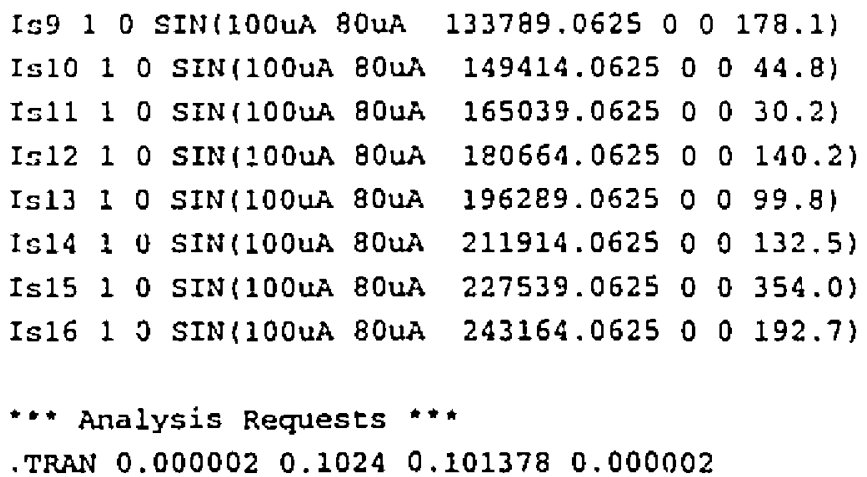

We then run HSPICE and use MATLAB to calculate the FFT of the output. The MATLAB file can be found in Section A.5. The result is plotted and is shown in Figure A3.

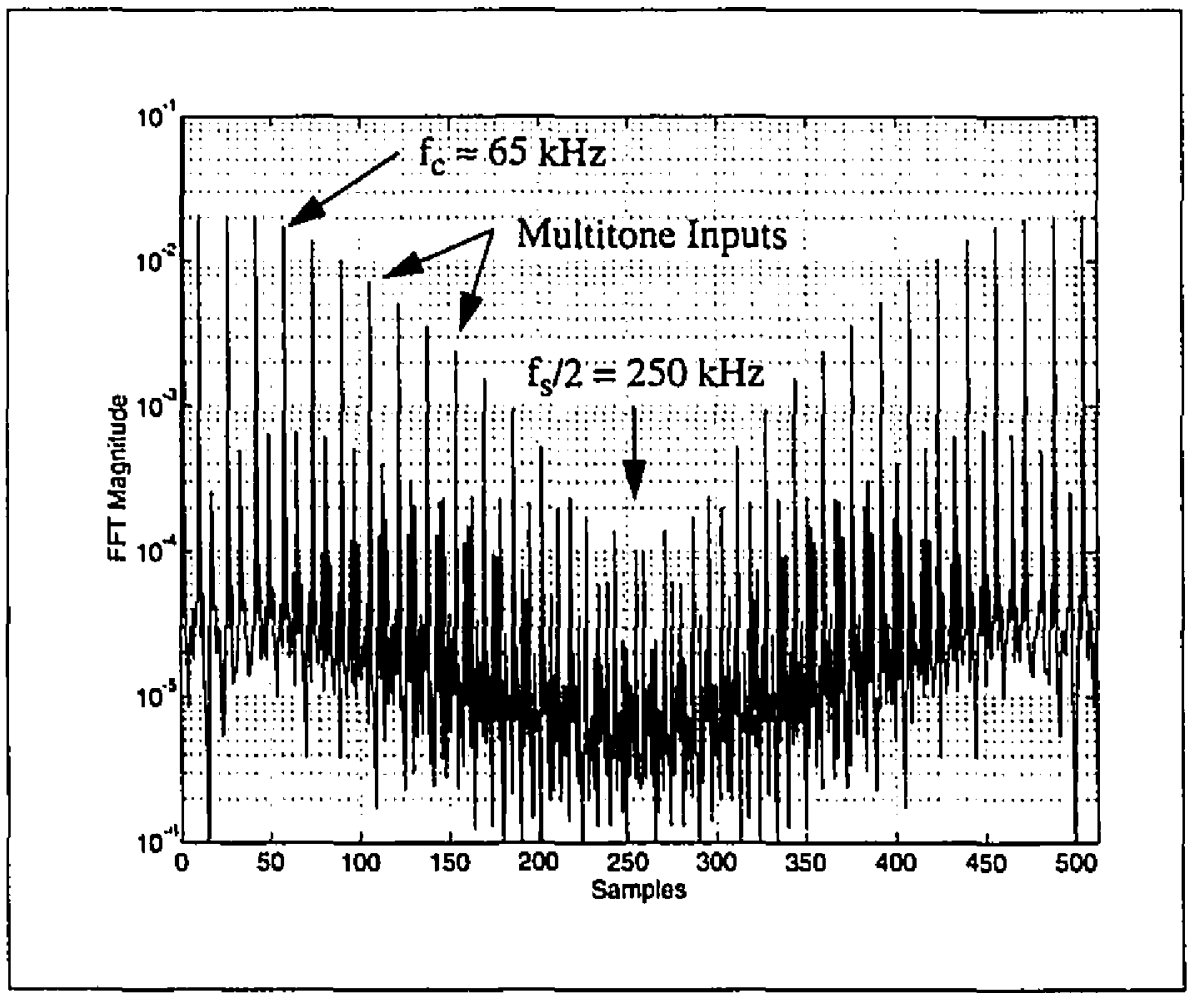

Figure A-3: FFT response of the log-domain biquad

The reader can clearly see the presence of the 16 tones of the multitone input. This allows us to verify the frequency response and measure the cutoff frequency, which is around $65 \mathrm{kHz}$, as expected. The smaller tones represent the many intermodulation 
products which occur when a multitone signal is applied to a circuit with non-linciarity present in it.

Overall we can see how this offers an excellent alternative for finding frequency information about non-linear circuits. By varying the number of tones at the input we cant get total harmonic distortion and intermodulation distortion results, as well as frepuency response.

\section{A.4 C Program for Generating the HSPICE File}

The $\mathrm{C}$ program used to generate the multitone inputs and the TRAN statement is reproduced below.

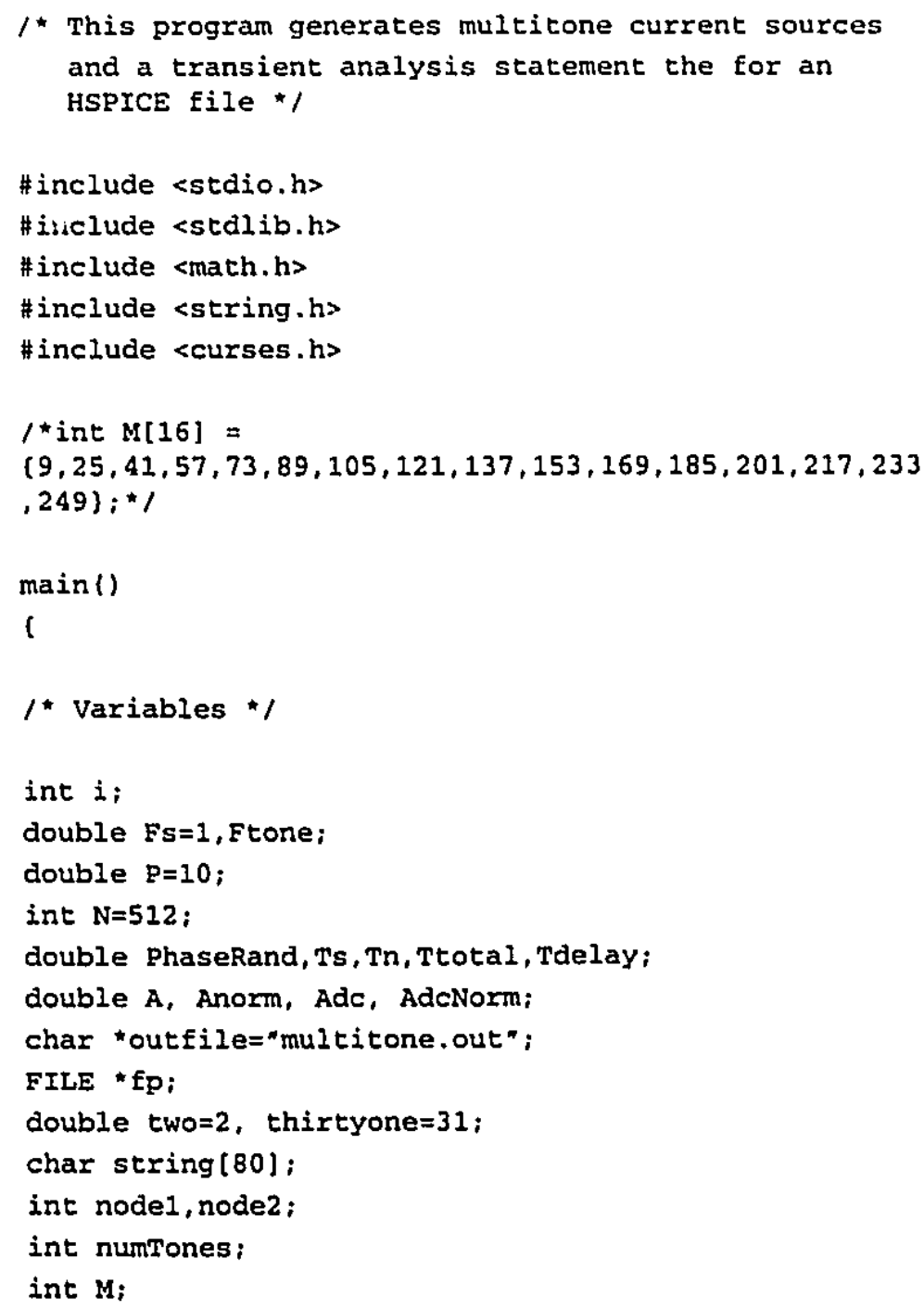




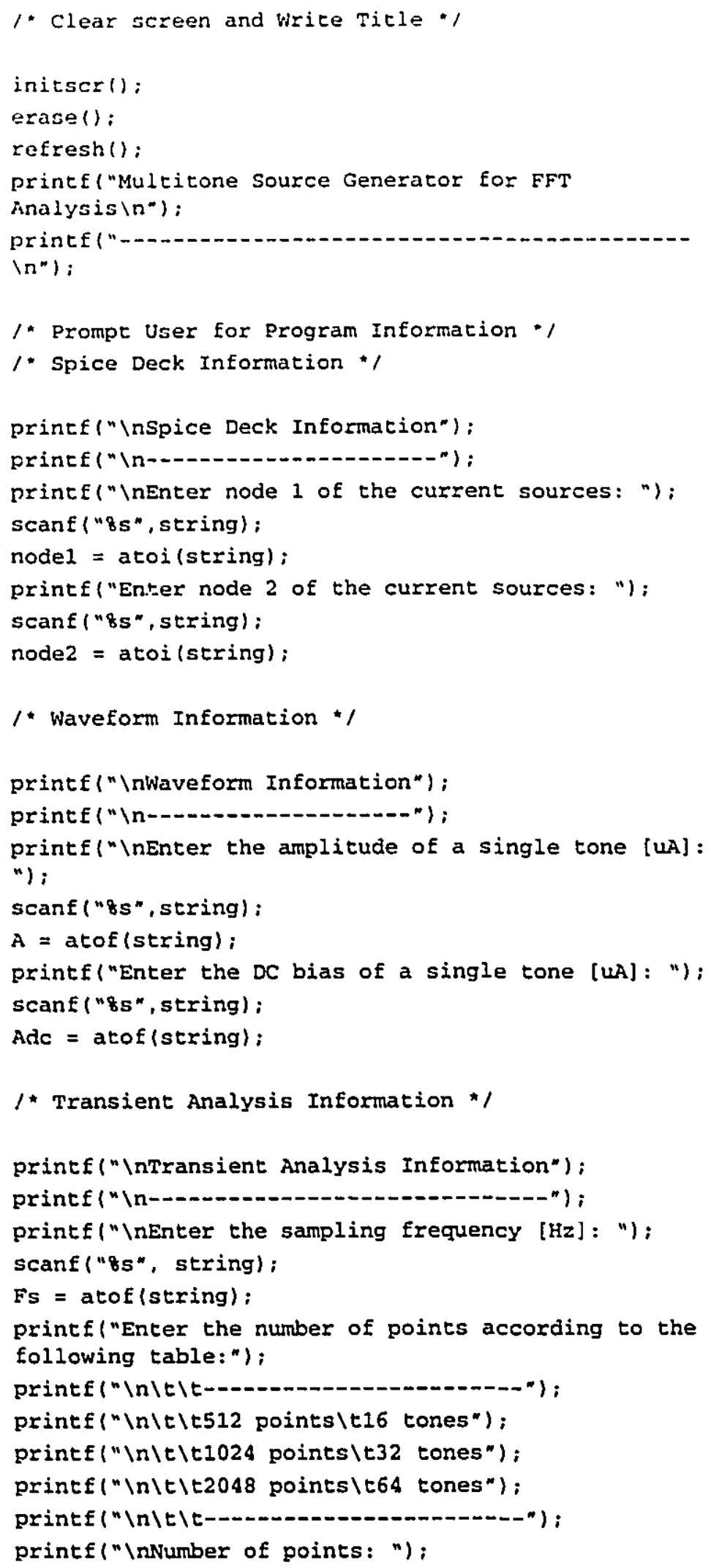




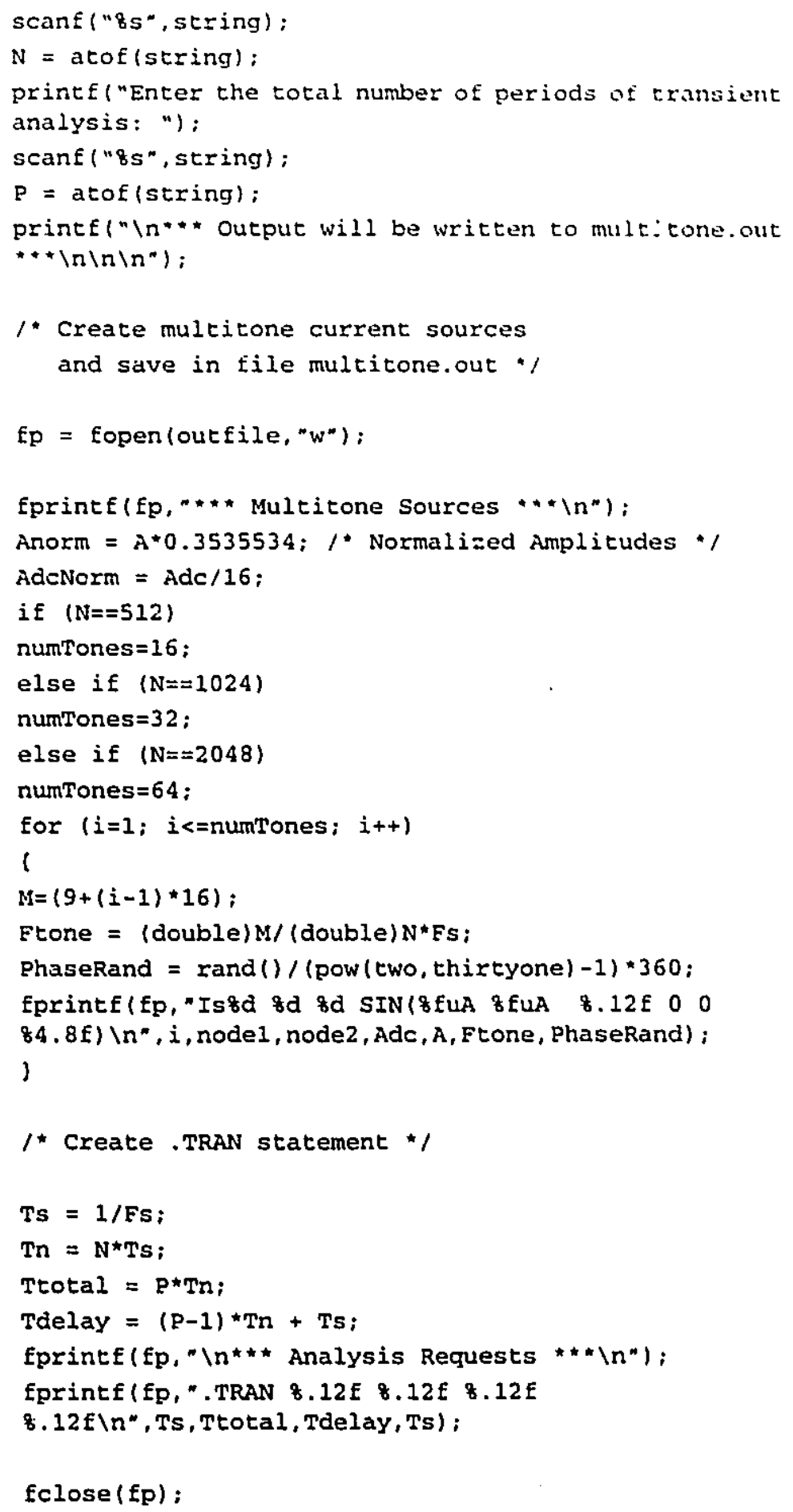




\section{A.5 MATLAB File for Calculating the FFT}

The following is the MATLAB file used to calculate the FFT and plot the result.

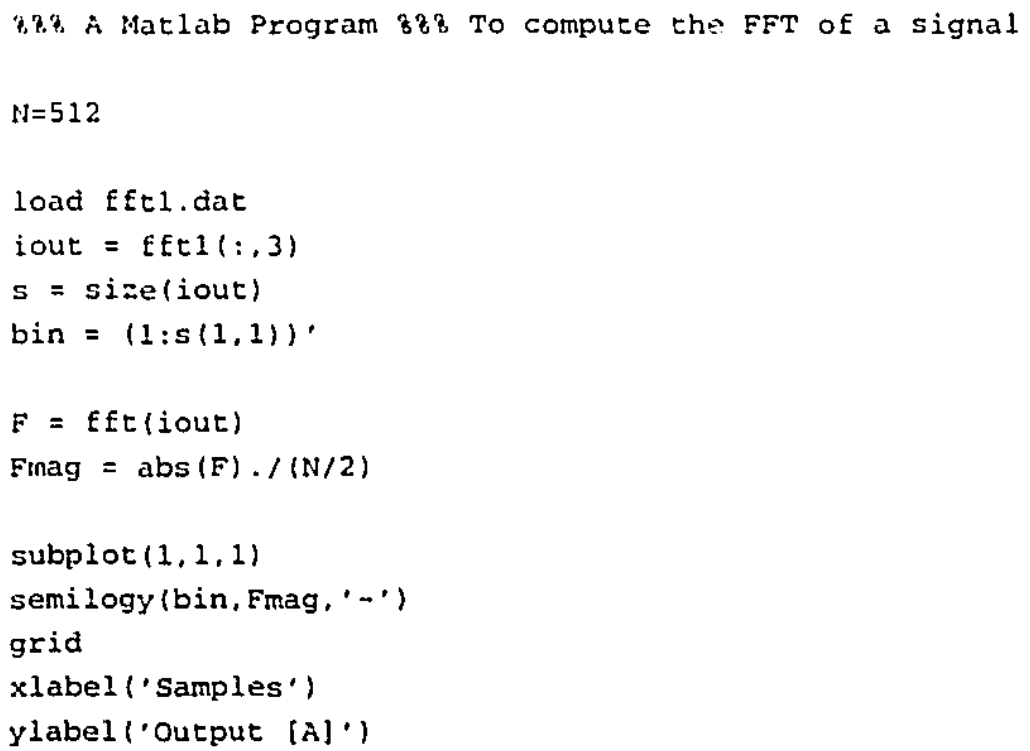




\section{References}

[1] Y. P. Tsividis and J. O. Voorman, Eds., Integrated Contimuous-Time Fillers, Piscataway, NJ: IEEE Press, 1993

[2] B. Gilbert, "Translinear circuits: a proposed classification", Electronics. Letters, vol. 11, pp. 14-16, Jan. 1975

[3] C. Toumazou, F. J. L.n gey and D. G. Haigh, Eds., Analogue IC Design: the current-mode approach, London, UK: Peter Peregrinus, 1990

[4] R. W. Adams, "Filtering in the log domain", Preprint \#1470 presented at the 63rd AES Conference, New York, NY, May 1979

[5] D. Frey, "Log domain filtering: an approach to current-mode filtering", IEE Proceedings, vol. 140, no. 6, pp. 406-416, Dec. 1993

[6] A. S. Sedra and P. O. Brackett, Filter Theory and Design: Active and Passive, Portland, OR: Matrix Publishers Inc., 1978

[7] A. S. Sedra an.1 K. C. Smith, Microelectronic Circuits, 3rd Edition, Florida, USA: Saunders College Publishing

[8] S. Sakurai, M. Ismail, J. Michel, E. Sanchez-Sinencio and R. Brannen, "A MOS$\mathrm{C}$ variable equalizer with simple on-chip automatic tuning", IEEE Journal of Solid-State Circuits, vol. 27, no. 6, pp. 927-934, June 1992

[9] J. van de: Plas, "MOSFET-C filter with low excess noise and accurate automatic tuning", IEEE Journal of Solid-State Circuits, vol. 26, no. 7, pp. 922-929, July 1991 
[10] M. Banu and Y. P. Tsividis, "An elliptic continuous-time CMOS filter with onchip automatic tuning", IEEE Journal of Solid-State Circuits, vol. 20, no. 6, pp. 1114-1121, Dec. 1985

111] U. Moon and B. Song, "A low-distortion $22 \mathrm{kHz}$ Sth-order Bessel filter", Proceedings from the 1993 IEEE Solid-State Circuits Conference, vol. 36, pp. 110-111, Feb. 1993

[12] A. B. Grebene, Bipolar and MOS Analog Integrated Circuit Design, New York, NY: John WIley and Sons, 1984

[13] W. M. Snelgrove and Ayal Shoval, "A balanced $0.9 \mu \mathrm{m}$ CMOS transconductanceC filter tunable over the VHF range", IEEE Journal of Solid-State Circuits, vol. 27, no. 3, pp. 314-323, March 1992

[14] B. Nauta, "A CMOS transconductance-C filter technique for very high frequencies", IEEE Journal of Solid-State Circuits, vol. 27, no. 2, pp. 142-153, Feb. 1992

[15] M. I. Ali, M. Howe, E. Sánchez-Sinencio and j. Ramírez-Angulo, "A BiCMOS low distortion tunable OTA for continuous-time filters", IEEE Transactions on Circuits and Systems I, vol. 40, no. 1, Jan. 1993

[16] J. Silva-Martinez, M. Steyaert and W. Sansen, "A large-signal very low-distortion transconductor for high-frequency continuous-time filters", IEEE Journal of Solid-State Circuits, vol. 26, pp. 946-955, July 1991

[17] S. D. Willingham and K. W. Martin, "A BiCMOS low-Distortion $8 \mathrm{MHz}$ lowpass filter", Proceedings from the 1993 IEEE Solid-State Circuits Conference, vol. 36 , pp. 114-115, Feb. 1993

[18] R. Schaumann and M. A. Tan, "The problem of on-chip automatic tuning in continuous-time integrated filters", Proceedings from the 1989 IEEE International Symposium on Circuits and Systems, pp. 106-109, Feb. 1989

[19] K. A. Kozma, D. A. Johns and A. S. Sedra, "Tuning of continuous-time filters in the presence of parasitic poles", IEEE Transactions on Circuits and Systems I, vol. 40, no. 1, Jan. 1993

[20] D. Frey, "A $3.3 \mathrm{~V}$ electronically tunable active filter usable to beyond $1 \mathrm{GHz}$ ", Proceedings from the 1994 IEEE International Symposium in Circuits and Systems, no. 5, pp. 493-496, June 1993

[21] M. L. Blostein, "Sensitivity analysis of parasitic effects in resistance terminated LC filters", IEEE Transactions on Circuit Theory, vol. CT-14, pp. 21-25, March 1967

[22] G. C. Temes and H. J. Orchard, "First order sensitivity and worst case analysis of doubly terminated reactance two-ports", IEEE Transactions on Circuit Theory, vol. CT-20, pp. 567-571, Sept. 1973 
[23] M. E. Van Valkenburg, Analog Filter Design, New York, NY:.Holt, Rinelaatt and Winston, 1982

[24] W. M. Snelgrove and A. S. Sedra, FILTOR 2 - A Computer Aided Filter De'sign Package, Champagne. Ill.: Matrix Publishers

[25] C. Ouslis, W. M. Snelgrove and A. S. Sedra, "A filter designer's filter design aid: filtorX", Proceedings of the IEEE International Symposium on Circuits and Sirstems, pp. 376-379, 1991

[26] O. Wing, "Ladder Network Analysis by Signal-Flow Graph - Appiication to Anilog Computer Programming", IRE Transactionss on Circuit Theory, vol. CT-3, pp. 289-294, Dec. 1956

[27] J. Vlach and K. Singhal, Computer Methods for Circuit Analysis and Design. New York, NY: Van Nostrand Reinhold, 1983

[28] G. W. Roberts, "A general classification of LC ladder based filter network synthesis methods", Course notes, McGill University, 1991

[29] Meta-Software, HSPICE User's Mamual, Vol. 1-3, Campbell, CA: Meta-Soltware, Inc., 1992

[30] G. W. Roberts and A. S. Sedra, SPICE for Microelectronic Circuits, 3rd Edition, Toronto, Canada: Saunders College Publishing, 1992

[31] Gennum Corporation, Gennum data book

[32] R. A. Witte, Spectrum and Network Measurements, NJ: Prentice-Hall, 1991

[33] D. Perry and G. W. Roberts, "Log-domain filters based on LC ladder synthesis", Proceedings of the 1995 IEEE International Symposium on Circuits and System., (to be presented ivlay 1995)

[34] M. Mahoney, DSP-Based Testing of Analog and Mixed-Signal Circuits, New York, NY: IEEE Computer Society Press, 1987

[35] J. G. Proakis and D. G. Manolakis, Digital Signal Processing, New York, NY: MacMillan Publishing Co., 1992

[36] P. Crawley and G. W. Roberts, "Predicting harmonic distortion in switched-current memory circuits", IEEE Transactions on Circuits and Systems, vol. 41, no. 2 , Feb. 1994 